\title{
Random sections of line bundles over real Riemann surfaces
}

\author{
Michele Ancona *
}

\begin{abstract}
Let $\mathcal{L}$ be a positive line bundle over a Riemann surface $\Sigma$ defined over $\mathbb{R}$. We prove that sections $s$ of $\mathcal{L}^{d}, d \gg 0$, whose number of real zeros $\# Z_{s}$ deviates from the expected one are rare. We also provide asymptotics of the form $\mathbb{E}\left[\left(\# Z_{s}-\mathbb{E}\left[\# Z_{s}\right]\right)^{k}\right]=O\left(\sqrt{d}^{k-1-\alpha}\right)$ and $\mathbb{E}\left[\# Z_{s}^{k}\right]=a_{k} \sqrt{d}^{k}+b_{k} \sqrt{d}^{k-1}+O\left(\sqrt{d}^{k-1-\alpha}\right)$ for all the (central) moments of the number of real zeros. Here, $\alpha$ is any number in $(0,1)$, and $a_{k}$ and $b_{k}$ are some explicit and positive constants. Finally, we obtain similar asymptotics for the distribution of complex zeros of random sections. Our proof involves Bergman kernel estimates as well as Olver multispaces.
\end{abstract}

\section{Contents}

1 Introduction $r$

2 Definitions and main tools $\quad 7$

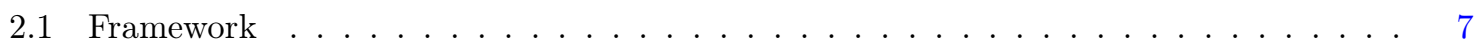

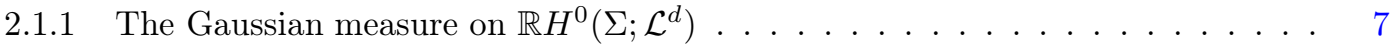

2.2 Bergman kernel . . . . . . . . . . . . . . . . . . . . . . . . 7

2.2.1 Exponential decay . . . . . . . . . . . . . . . . . . . 8

2.2 .2 Normal coordinates . . . . . . . . . . . . . . . . . . . 8

2.2 .3 Near diagonal estimate . . . . . . . . . . . . . . . . . . . . . . . 8

2.2.4 Scaled Bergman kernel . . . . . . . . . . . . . . . . . . . . 9

3 Incidence manifold and density function $\quad 9$

3.1 Zeros of random real sections . . . . . . . . . . . . . . . . . . . . . . . 9

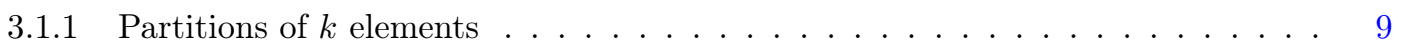

3.1 .2 Modified moments . . . . . . . . . . . . . . . . . . . 10

3.2 Incidence manifold and density function $\mathcal{R}_{d}^{k} \ldots \ldots \ldots \ldots \ldots$

3.2.1 Incidence manifold . . . . . . . . . . . . . . . . . . . . . . . 10

3.2 .2 The density function $\mathcal{R}_{d}^{k} \ldots \ldots \ldots \ldots \ldots \ldots \ldots$

4 Proof of the main theorems $\quad 13$

4.1 Uniform bound on the $L^{\infty}$-norm of the density function $\mathcal{R}_{d}^{k} \ldots \ldots \ldots$. . . . . . . 13

4.2 Proof of Theorems 1.1, 1.4 and $1.5 \ldots \ldots \ldots \ldots$

5 Olver multispaces $\quad 18$

5.1 Olver multispaces . . . . . . . . . . . . . . . . . . . . . . . 18

5.1 .1 Pointed manifolds . . . . . . . . . . . . . . . . . . . . 18

5.1 .2 Multi-contact . . . . . . . . . . . . . . . . . . . . 19

5.1 .3 Divided differences . . . . . . . . . . . . . . . . . . . . 19

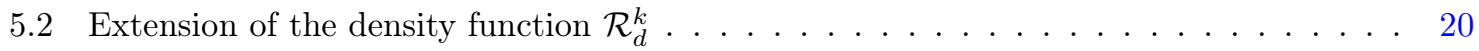

5.2.1 Extension of the incidence manifold . . . . . . . . . . . . . . . . . 20

5.3 Local equations of the incidence manifold . . . . . . . . . . . . . . . . . . . . 20

5.3 .1 A partition of $\mathbb{R} \Sigma^{k} \ldots \ldots \ldots \ldots \ldots$

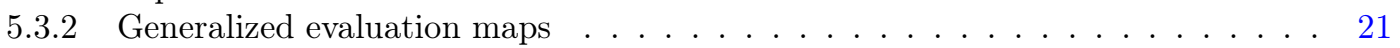

5.3.3 New expressions for the density function $\mathcal{R}_{d}^{k} \ldots \ldots \ldots \ldots$. . . . . . . . . 22

5.3 .4 Proof of Theorem $4.1 \ldots \ldots \ldots \ldots \ldots$

5.4 Estimates of the denominator $\mathcal{D}_{d}^{\Gamma} \ldots \ldots \ldots \ldots \ldots \ldots \ldots \ldots$

*Institut Camille Jordan, Umr Cnrs 5208, Université Claude Bernard Lyon 1. ancona@math.univ-lyon1.fr 
5.4.1 A partition of $\mathbb{R}^{k} \ldots \ldots \ldots \ldots \ldots \ldots 24$

5.4 .2 A local evaluation model . . . . . . . . . . . . . . . . . 24

5.4 .3 Local boundedness results . . . . . . . . . . . . . . . . . . . . . 25

5.4 .4 Generalized evaluation maps in normal coordinates . . . . . . . . . . . . . . . 27

5.4 .5 Scaled evaluation maps . . . . . . . . . . . . . . . . . . . . 27

5.4 .6 Reduction to the local model . . . . . . . . . . . . . . . . . . . . . 28

5.5 Estimates of the numerator $\mathcal{N}_{d}^{\Gamma} \ldots \ldots \ldots \ldots \ldots \ldots \ldots \ldots$. . . . . . . . . . . . . . . 28

5.6 Off-diagonal estimates of the density function and proof of Proposition 4.7 . . . . . . 30

5.6.1 About the mean and the variance. . . . . . . . . . . . . . 30

5.6 .2 Estimates of the density function . . . . . . . . . . . . . . . . . 32

6 Complex case $r$

6.1 The complex random setting . . . . . . . . . . . . . . . . . . . . . 33

6.1.1 The Gaussian measure on $H^{0}\left(\Sigma ; \mathcal{L}^{d}\right) \ldots \ldots \ldots$. . . . . . . . . . . . . . . . . . . 33

6.2 Olver holomorphic multispace . . . . . . . . . . . . . . . . . . . . . 33

6.3 Higher moments for random holomorphic sections . . . . . . . . . . . . . . . . . . 33

\section{Introduction}

Let $p \in \mathbb{R}_{d}[X]$ be a degree $d$ polynomial with real coefficients.

How many real roots does $p$ have, choosing it at random?

A first answer was given by Kac in the 40 's. He proved that the expected number of real roots $\mathbb{E}\left[\# Z_{p}\right]$ is equivalent to $\frac{2}{\pi} \log d$ as $d$ grows to $+\infty$, see [13]. By definition,

$$
\mathbb{E}\left[\# Z_{p}\right]=\int_{p \in \mathbb{R}_{d}[X]}\left(\# Z_{p}\right) d \mu(p)
$$

where $\# Z_{p}$ denotes the cardinality of $Z_{p}=\{x \in \mathbb{R} \mid p(x)=0\}$. The probability measure on $\mathbb{R}_{d}[X] \mathrm{Kac}$ has considered was the Gaussian measure associated with a scalar product that makes $\left\{X^{k}\right\}_{0 \leqslant k \leqslant d}$ an orthonormal basis.

In the 90's, another answer was given by Kostlan and by Shub and Smale. They proved that $\mathbb{E}\left[\# Z_{p}\right]=\sqrt{d}$ for any $d \in \mathbb{N}$, see $[14,22]$. There, the probability measure on $\mathbb{R}_{d}[X]$ was the Gaussian measure associated with a scalar product that turns $\left\{\sqrt{\left(\begin{array}{c}d \\ k\end{array}\right)} X^{k}\right\}_{0 \leqslant k \leqslant d}$ into an orthonormal basis. This scalar product makes $\mathbb{R}_{d}[X]$ isometric to the space $\mathbb{R} H^{0}\left(\mathbb{C} P^{1} ; \mathcal{O}(d)\right)$ of real global sections of the line bundle $\mathcal{O}(d)$ over $\mathbb{C} P^{1}$, where the scalar product on $\mathbb{R} H^{0}\left(\mathbb{C} P^{1} ; \mathcal{O}(d)\right)$ is the $L^{2}$-scalar product induced by the Fubini-Study metric on $\mathcal{O}(d)$, see Section 2.1. This point of view brings us to consider a general Riemann surface $\Sigma$ with any ample line bundle over $\Sigma$, see $[10,11,20]$.

Let $\Sigma$ be a closed real Riemann surface, that is a closed Riemann surface equipped with an antiholomorphic involution $c_{\Sigma}$. We denote by $\mathbb{R} \Sigma=\operatorname{Fix}\left(c_{\Sigma}\right)$ its real locus. Let $\mathcal{L}$ be a real ample line bundle over $\Sigma$, that is an ample holomorphic line bundle $p: \mathcal{L} \rightarrow \Sigma$ equipped with an anti-holomorphic involution $c_{\mathcal{L}}$ such that $p \circ c_{\mathcal{L}}=c_{\Sigma} \circ p$ and $c_{\mathcal{L}}$ is complex-antilinear in the fibers. A real Hermitian metric on $\mathcal{L}$ is a Hermitian metric $h$ such that $c_{\mathcal{L}}^{*} h=\bar{h}$.

A real Hermitian metric on $\mathcal{L}$ induces a $L^{2}$-scalar product, and thus a Gaussian measure, on the space of global holomorphic real sections of $\mathcal{L}^{d}$, see Section 2.1. We denote such space by $\mathbb{R} H^{0}\left(\Sigma ; \mathcal{L}^{d}\right) \doteqdot$ $\left\{s \in H^{0}(\Sigma ; \mathcal{L}) \mid c_{L} \circ s=s \circ c_{\Sigma}\right\}$.

$$
\text { How many real zeros does a random real section } s \in \mathbb{R} H^{0}\left(\Sigma ; \mathcal{L}^{d}\right) \text { have? }
$$

We denote by $Z_{s}=\{s=0\} \cap \mathbb{R} \Sigma$ the set of real zeros of $s \in \mathbb{R} H^{0}\left(\Sigma ; \mathcal{L}^{d}\right)$. In [11] it is shown that $\lim _{d \rightarrow \infty} \frac{1}{\sqrt{d}} \mathbb{E}\left[\# Z_{s}\right]=\frac{\operatorname{Vol}_{h}(\mathbb{R} \Sigma)}{\sqrt{\pi}}$. We get

Theorem 1.1. Let $c(d) \in \mathbb{R}_{+}^{*}$ be any sequence of positive real numbers, then, for any $k \in \mathbb{N}$ and any $\alpha \in(0,1)$, the following holds as $d \rightarrow \infty$ :

$$
\text { - } \mu\left\{\left|\# Z_{s}-\mathbb{E}\left[\# Z_{s}\right]\right|>c(d) \sqrt{d}\right\}=O\left(\frac{1}{c(d)^{k} \sqrt{d}^{1+\alpha}}\right) ;
$$


- for any measurable subset $A \subset \mathbb{R} \Sigma$ of positive volume:

$$
\mu\left\{\left|\#\left(Z_{s} \cap A\right)-\mathbb{E}\left[\#\left(Z_{s} \cap A\right)\right]\right|>c(d) \sqrt{d}\right\}=O\left(\frac{\log d}{c(d)^{k} \sqrt{d}}\right) .
$$

The measure of the set of real sections whose number of real zeros deviates from the expected one goes to zero faster than all polynomials. Moreover, this property is valid for any open subset of $\mathbb{R} \Sigma$.

Remark 1.2. For a real analytic metric $h$, it seems possible to improve the polynomial upper estimate of Theorem 1.1 by an exponential one. This is a work in progress. Recall that real analytic metrics are dense in the space of smooth metrics (see [6, 23]) and that the Fubini-Study metric on $\mathbb{C} P^{1}$ is real analytic. Remark that an exponential rarefaction for real sections with "many" real zeros is proved in [10, Theorem 2]

Higher moments The main ingredient of the proof of Theorem 1.1 is an estimate of all the moments $\mathbb{E}\left[\# Z_{s}^{k}\right], k \in \mathbb{N}$, which has its own interest. By definition, the $k$-th moment of $\# Z_{s}$ equals

$$
\mathbb{E}\left[\# Z_{s}^{k}\right]=\int_{s \in \mathbb{R} H^{0}\left(\Sigma ; \mathcal{L}^{d}\right)}\left(\# Z_{s}\right)^{k} d \mu(s) .
$$

The computation of $\mathbb{E}\left[\# Z_{s}^{k}\right]$ is actually the core of the paper.

Theorem 1.3. There exists a universal positive constant $M$ such that, for any positive real Hermitian line bundle $(\mathcal{L}, h)$ over any real Riemann surface $\Sigma$, any integer $k$ and any $\alpha \in(0,1)$, the following asymptotics hold:

$$
\frac{1}{\sqrt{d}^{k}} \mathbb{E}\left[\# Z_{s}^{k}\right]=\frac{\operatorname{Vol}_{h}(\mathbb{R} \Sigma)^{k}}{\sqrt{\pi}^{k}}+\frac{M k(k-1) \operatorname{Vol}_{h}(\mathbb{R} \Sigma)^{k-1}}{2 \sqrt{\pi}^{k-2} \sqrt{d}}+O\left(\frac{1}{\sqrt{d}^{1+\alpha}}\right) .
$$

By universal constant we mean that it is a constant which neither depends on $\Sigma$ nor on $k \in \mathbb{N}$. This formula was known for $k=1$ and $k=2$ (see resp. [11, Theorem 1.1] or [15, Theorem 1.3] and [16, Theorem 1.6]) and unknown in this general case. Theorem 1.3 is a consequence of a more precise equidistribution result, namely Theorem 1.4 below. Before stating this, we need some definitions.

- For all $s \in \mathbb{R} H^{0}\left(\Sigma ; \mathcal{L}^{d}\right)$, consider the empirical measure

$$
\nu_{s}=\sum_{x \in Z_{s}} \delta_{x}
$$

where $\delta_{x}$ is the Dirac measure at $x$. It induces a measure $\nu_{s}^{k}$ on $\mathbb{R} \Sigma^{k}$ defined, for every continuous function $f \in C^{0}\left(\mathbb{R} \Sigma^{k}\right)$, by

$$
\nu_{s}^{k}(f)=\sum_{\left(x_{1}, \ldots, x_{k}\right) \in Z_{s}^{k}} f\left(x_{1}, \ldots, x_{k}\right)
$$

- The curvature form $\omega$ of $(\mathcal{L}, h)$ gives rise to the Kähler metric on $\Sigma$ which induces a Riemannian metric on the real locus $\mathbb{R} \Sigma$. We denote by $\left|\mathrm{dV}_{h}\right|^{k}$ the induced Riemannian volume form on $\mathbb{R} \Sigma^{k}$.

- For any $1 \leq a<b \leq k$, we denote by $j_{a b}: \mathbb{R} \Sigma^{k-1} \hookrightarrow \mathbb{R} \Sigma^{k}$ the inclusion

$$
\left(x_{1}, \ldots, x_{a}, \ldots, \hat{x}_{b}, \ldots, x_{k}\right) \mapsto\left(x_{1}, \ldots, x_{a}, \ldots, x_{a}, \ldots, x_{k}\right),
$$

so that the image $j_{a b}\left(\mathbb{R} \Sigma^{k-1}\right)$ is equal to $\left\{\left(x_{1}, \ldots, x_{k}\right) \in \mathbb{R} \Sigma^{k} \mid x_{a}=x_{b}\right\}$.

- Finally, for any $f \in C^{0}\left(\mathbb{R} \Sigma^{k}\right)$, we denote by $\omega_{f}$ its modulus of continuity, that is $\omega_{f}: \epsilon \in \mathbb{R}_{+}^{*} \mapsto$ $\sup \left\{|f(x)-f(y)|, \mathbf{d}_{h}(x, y) \leq \epsilon\right\} \in \mathbb{R}_{+}$, where $\mathbf{d}_{h}$ is the Riemannian distance on $\mathbb{R} \Sigma$ induced by the real Hermitian metric $h$. 
Theorem 1.4. There exists a universal positive constant $M$ such that, for any positive real Hermitian line bundle $(\mathcal{L}, h)$ over any real Riemann surface $\Sigma$, any $k \in \mathbb{N}$ and any $f: \mathbb{R} \Sigma^{k} \rightarrow \mathbb{R}$ bounded function, the following asymptotics hold:

$$
\frac{1}{\sqrt{d}^{k}} \mathbb{E}\left[\nu_{s}^{k}\right](f)=\frac{1}{\sqrt{\pi}^{k}} \int_{\mathbb{R} \Sigma^{k}} f\left|d V_{h}\right|^{k}+O\left(\frac{\log d}{\sqrt{d}}\right) .
$$

Moreover, if $f$ is continuous, we have:

$$
\frac{1}{\sqrt{d}^{k}} \mathbb{E}\left[\nu_{s}^{k}\right](f)=\frac{1}{\sqrt{\pi}^{k}} \int_{\mathbb{R} \Sigma^{k}} f\left|d V_{h}\right|^{k}+\frac{M}{\sqrt{\pi}^{k-2} \sqrt{d}} \sum_{1 \leq a<b \leq k} \int_{\mathbb{R} \Sigma^{k-1}} j_{a b}^{*} f\left|d V_{h}\right|^{k-1}+o\left(\frac{1}{\sqrt{d}}\right) .
$$

The error term $o\left(\frac{1}{\sqrt{d}}\right)$ is bounded from above by

$$
\|f\|_{\infty}\left(O\left(\frac{1}{\sqrt{d}^{1+\alpha}}\right)+\omega_{f}\left(\frac{1}{\sqrt{d}^{\alpha}}\right) O\left(\frac{1}{\sqrt{d}}\right)\right)
$$

for any $\alpha \in(0,1)$, where $\omega_{f}(\cdot)$ is the modulus of continuity of $f$. Moreover, the errors $O\left(\frac{1}{\sqrt{d}^{1+\alpha}}\right)$, $O\left(\frac{1}{\sqrt{d}}\right)$ do not depend on $f$.

Choosing $f=1$ in Theorem 1.4, we obtain Theorem 1.3.

We also investigate the asymptotic behaviour of the central moments. We define the $k$-th central moment of the random variable $\nu_{s}$ as $\mathbb{E}\left[\left(\nu_{s}-\mathbb{E}\left[\nu_{s}\right]\right)^{k}\right]$, where, for all $f \in C^{0}(\mathbb{R} \Sigma)$,

$$
\mathbb{E}\left[\left(\nu_{s}-\mathbb{E}\left[\nu_{s}\right]\right)^{k}\right](f)=\int_{s \in \mathbb{R} H^{0}\left(\Sigma ; \mathcal{L}^{d}\right)}\left(\nu_{s}(f)-\mathbb{E}\left[\nu_{s}\right](f)\right)^{k} d \mu(s) .
$$

Theorem 1.5. Under the hypothesis of Theorem 1.3, for all $f: \mathbb{R} \Sigma \rightarrow \mathbb{R}$ bounded function and any $k>2$, the following holds as $d \rightarrow \infty$ :

$$
\frac{1}{\sqrt{d}^{k}} \mathbb{E}\left[\left(\nu_{s}-\mathbb{E}\left[\nu_{s}\right]\right)^{k}\right](f)=O\left(\frac{\log d}{\sqrt{d}}\right) .
$$

Moreover, if $f$ is continuous, we have

$$
\frac{1}{\sqrt{d}^{k-1}} \mathbb{E}\left[\left(\nu_{s}-\mathbb{E}\left[\nu_{s}\right]\right)^{k}\right](f)=o(1) .
$$

The term o(1) is bounded from above by

$$
\|f\|_{\infty}\left(O\left(\frac{1}{\sqrt{d}^{\alpha}}\right)+\omega_{f}\left(\frac{1}{\sqrt{d}^{\alpha}}\right) O(1)\right)
$$

for any $\alpha \in(0,1)$, where $\omega_{f}(\cdot)$ is the modulus of continuity of $f$. Moreover, the errors $O\left(\frac{1}{\sqrt{d}^{\alpha}}\right)$ and $O(1)$ do not depend on $f$.

In particular, for $f=1$, we get $\mathbb{E}\left[\left(\# Z_{s}-\mathbb{E}\left[\# Z_{s}\right]\right)^{k}\right]=O\left(\sqrt{d}^{k-1-\alpha}\right)$. In Theorem 1.5 we put the hypothesis $k>2$ in order to have a more concise formula. In fact, the moment $\mathbb{E}\left[\left(\# Z_{s}-\mathbb{E}\left[\# Z_{s}\right]\right)^{k}\right]$ has a different behavior in the case $k=1$ or 2 . For $k=1$ it is trivially 0 . For $k=2$, which corresponds to the variance $\operatorname{Var}\left(\# Z_{s}\right)$, it has been proved that it grows as $\sqrt{d}$, see [16].

Case of random polynomials Let $p \in \mathbb{R}_{d}[X]$ be a degree $d$ real polynomial and $Z_{p}=\{x \in \mathbb{R} \mid$ $p(x)=0\}$ be the real zeros of $p$. We equip $\mathbb{R}_{d}[X]$ with the Gaussian measure $\mu$ associated with the scalar product that makes $\left\{\sqrt{\left(\begin{array}{c}d \\ k\end{array}\right)} X^{k}\right\}_{0 \leqslant k \leqslant d}$ an orthonormal basis. The probability space $\left(\mathbb{R}_{d}[X], \mu\right)$ is called the Kostlan-Shub-Smale model.

Corollary 1.6. Let $c(d) \in \mathbb{R}_{+}^{*}$ be any sequence of real numbers, then for any $k>2$ and any $\alpha \in(0,1)$, the following holds as $d \rightarrow \infty$ : 
- $\mu\left\{\left|\# Z_{p}-\sqrt{d}\right|>c(d) \sqrt{d}\right\}=O\left(\frac{1}{c(d)^{k} \sqrt{d}^{1+\alpha}}\right)$;

- for any measurable subset $A \subset \mathbb{R} P^{1}$ of positive volume:

$$
\mu\left\{\left|\#\left(Z_{p} \cap A\right)-\mathbb{E}\left[\#\left(Z_{p} \cap A\right)\right]\right|>c(d) \sqrt{d}\right\}=O\left(\frac{\log d}{c(d)^{k} \sqrt{d}}\right) .
$$

Corollary 1.7. Let $p \in\left(\mathbb{R}_{d}[X], \mu\right)$ be a random Kostlan-Shub-Smale polynomial. There exists a positive constant $C$ such that for any $k \in \mathbb{N}$ and any $\alpha \in(0,1)$ the following asymptotics hold

$$
\begin{gathered}
\mathbb{E}\left[\# Z_{p}^{k}\right]=\sqrt{d}^{k}+C k(k-1) \sqrt{d}^{k-1}+O\left(\sqrt{d}^{k-1-\alpha}\right) \\
\mathbb{E}\left[\left|\# Z_{p}-\sqrt{d}\right|^{k}\right]=O\left(\sqrt{d}^{k-1-\alpha}\right) .
\end{gathered}
$$

A Kostlan-Shub-Smale polynomial $p \in\left(\mathbb{R}_{d}[X], \mu\right)$ is in fact a real random section of $\mathcal{O}_{\mathbb{C} P^{1}}(d) \rightarrow$ $\mathbb{C} P^{1}$, where we equip $\mathcal{O}_{\mathbb{C} P^{1}}(1)$ and hence $\mathcal{O}_{\mathbb{C} P^{1}}(d)$ with the Fubini Study metric. The corollaries follow from Theorems 1.1, 1.3 and 1.5 and from the fact that the Fubini-Study volume of $\mathbb{R} P^{1}$ is equal to $\sqrt{\pi}$. The constant $C$ appearing in Corollary 1.7 is then equal to $\frac{M \sqrt{\pi}}{2}$, where $M$ is the universal constant of Theorem 1.4. Corollary 1.7 for $k=2$ has already been proved in [8], in which a Central Limit Theorem for Kostlan-Shub-Smale polynomial is also shown (see also [2] for the variance of the number of solutions of a system of random polynomials). However, also for the case of random polynomials, our proof differs from the one of [8].

Complex case These techniques can be applied also in the complex case. For all $s \in H^{0}\left(\Sigma ; \mathcal{L}^{d}\right)$, let $C_{s}$ be the current of integration over $\{s=0\}$, that is the empirical measure $C_{s}(f)=\sum_{x \in\{s=0\}} f(x)$.

How do the zeros of a random section distribute over $\Sigma$ ?

It is known that $\frac{1}{d} \mathbb{E}\left[C_{s}\right]=\omega+O\left(\frac{1}{d}\right)$, where $\omega$ is the curvature form of $h$, see [20, Proposition 3.2]. In [5, Theorem 3.4], the correlations between simultaneous zeros of a random section is proved to be indipendent of the line bundle $\mathcal{L}^{d}$. The main result in the complex setting is the following:

Theorem 1.8. Let $(\mathcal{L}, h)$ be a positive Hermitian line bundle over a Riemann surface $\Sigma$ and let $\omega$ be the Kähler curvature form of $h$. Then, for all $k \in \mathbb{N}$ and for all $f \in C^{0}\left(\Sigma^{k}\right)$, the following asymptotics hold:

$$
\frac{1}{d^{k}} \mathbb{E}\left[C_{s}^{k}\right](f)=\int_{\Sigma^{k}} f d V_{\omega}^{k}+\|f\|_{\infty} O\left(\frac{1}{d}\right)
$$

where $d V_{\omega}^{k}$ is the volume form on $\Sigma^{k}$ induced by $\omega$. $1.1]$.

For $k=2$, a more precise result has been obtained by Shiffman and Zelditch, see [21, Theorem

Idea of the proof The pattern of the proof is the following

Theorem $1.4 \Rightarrow$ Theorem $1.5 \Rightarrow$ Theorem 1.1 .

The first implication is proved by simple algebraic operations, the second one using the classical Markov inequality. Theorem 1.4 is then the core of the paper. The proof of Theorem 1.4 is of geometric nature. Using Kac-Rice formula, we write the moment $\mathbb{E}\left[\nu_{s}^{k}\right](f)$ as an integral over $\mathbb{R} \Sigma^{k}$ of the form

$$
\int_{\underline{x} \in \mathbb{R} \Sigma^{k}} f(\underline{x}) \mathcal{R}_{d}^{k}(\underline{x})\left|\mathrm{d} \mathrm{V}_{h}\right|^{k} .
$$

Here, $\mathcal{R}_{d}^{k}$ is a smooth function defined on $\mathbb{R} \Sigma^{k} \backslash \Delta$, where $\Delta$ is the diagonal of $\mathbb{R} \Sigma^{k}$. By standard techniques, we can write, for any $\underline{x}=\left(x_{1}, \ldots, x_{k}\right) \in \mathbb{R} \Sigma^{k} \backslash \Delta$,

$$
\mathcal{R}_{d}(\underline{x})=\frac{\mathcal{N}_{d}^{k}(\underline{x})}{\mathcal{D}_{d}^{k}(\underline{x})}
$$


The functions $\mathcal{N}_{d}^{k}$ and $\mathcal{D}_{d}^{k}$ are explicit and given in Proposition 3.9. In particular, using Bergman kernel estimates, we show that $\mathcal{R}_{d}^{k}$ locally grows as $\sqrt{d}^{k}$. The denominator $\mathcal{D}_{d}^{k}(\underline{x})$ is the normal Jacobian of the evaluation map

$$
e v_{\underline{x}}: s \in \mathbb{R} H^{0}\left(\Sigma ; \mathcal{L}^{d}\right) \mapsto\left(s\left(x_{1}\right), \ldots, s\left(x_{k}\right)\right) \in \mathbb{R} \mathcal{L}_{x_{1}}^{d} \times \cdots \mathbb{R} \mathcal{L}_{x_{k}}^{d} .
$$

It equals the square root of the determinant of a symmetric $n \times n$ matrix whose $(i, j)$-entry is $\mathcal{K}_{d}\left(x_{i}, x_{j}\right)$, where $\mathcal{K}_{d}$ is the Bergman kernel of the line bundle $\mathcal{L}^{d}$. This normal Jacobian vanishes on the diagonal $\Delta$ of $\mathbb{R} \Sigma^{k}$ and this is the reason why $\mathcal{R}_{d}^{k}$ is (a priori) defined only over $\mathbb{R} \Sigma^{k} \backslash \Delta$. The main steps for studying the function $\mathcal{R}_{d}^{k}$ are the following:

1. Outside a neighborhood $\Delta_{d}$ of the diagonal $\Delta$ of size around $\frac{\log d}{\sqrt{d}}$ we have

$$
\frac{1}{\sqrt{d}^{k}} \mathcal{R}_{d}^{k}\left(x_{1}, \ldots, x_{k}\right)=\prod_{i=1}^{k} \frac{1}{\sqrt{d}} \mathcal{R}_{d}^{k}\left(x_{i}\right)+O\left(\frac{1}{d}\right)=\frac{1}{\sqrt{\pi}^{k}}+O\left(\frac{1}{d}\right)
$$

where the error term is uniform in $\left(x_{1}, \ldots, x_{k}\right) \in \mathbb{R} \Sigma^{k} \backslash \Delta_{d}$. This is essentially due to the fact that we can express $\mathcal{R}_{d}^{k}\left(x_{1}, \ldots, x_{k}\right)$ in terms of the Bergman kernel $\mathcal{K}_{d}$ at points $\left(x_{i}, x_{j}\right), i, j \in$ $\{1, \ldots, k\}$, and that $\frac{1}{d} \mathcal{K}_{d}\left(x_{i}, x_{j}\right)$ uniformly goes to zero as $\mathbf{d}_{h}\left(x_{i}, x_{j}\right)>\frac{\log d}{\sqrt{d}}$, see Proposition 4.3 .

2. Using Olver multispaces we are able to extend the function $\mathcal{R}_{d}^{k}$ over all the compact manifold $\mathbb{R} \Sigma^{k}$. Then, a careful analysis of $\mathcal{R}_{d}^{k}$ in a neighborhood of the diagonal and the compactness of $\mathbb{R} \Sigma^{k}$ will give us an uniform boundedness of $\frac{1}{\sqrt{d}^{k}} \mathcal{R}_{d}^{k}$, that is there exists a constant $C$ such that $\frac{1}{\sqrt{d}^{k}} \mathcal{R}_{d}^{k}(\underline{x})<C$ for every $\underline{x} \in \mathbb{R} \Sigma^{k}$ and every $d$. This is the content of Theorem 4.1.

3. At this level we are able to prove that

$$
\frac{1}{\sqrt{d}^{k}} \int_{\mathbb{R} \Sigma^{k}} f \mathcal{R}_{d}^{k}\left|\mathrm{dV}_{h}\right|^{k}=\frac{1}{\sqrt{\pi}^{k}} \int_{\mathbb{R} \Sigma^{k}} f\left|\mathrm{dV}_{h}\right|^{k}+\|f\|_{\infty} O\left(\frac{\log d}{\sqrt{d}}\right) .
$$

The first term of the right hand side is given by integrating over the complement of the neighborhood $\Delta_{d}$ of the diagonal and by using point 1 . The error term is given by the integral over $\Delta_{d}$. This integral is bounded from above by the product of the volume of $\Delta_{d}$, that is a $O\left(\frac{\log d}{\sqrt{d}}\right)$, times the infinity norms of $f$ and of $\frac{1}{\sqrt{d}^{k}} \mathcal{R}_{d}^{k}(\underline{x})$. The latter is finite thanks to point 2 above.

4. Finally, a more careful analysis of $\mathcal{R}_{d}^{k}$ in a neighborhood of the diagonal will give us also the second term in the asymptotic expansion of Theorem 1.4. For this, we introduce some subsets $U_{d}^{a, b}$ of $\mathbb{R} \Sigma^{k}, 1 \leq a<b \leq k$, which, roughly speaking, are the set of $\left(x_{1}, \ldots, x_{k}\right)$ such that the distance between every pair of points $\left(x_{i}, x_{j}\right), i \neq j \in\{1, \ldots, k\}$, is bigger than $\frac{\log d}{\sqrt{d}}$, except at most the pair $\left(x_{a}, x_{b}\right)$, see Definition 4.5. With similar techniques as in the point 1 above, we are able to estimate $\mathcal{R}_{d}^{k}$ is these subsets, see Propositions 4.3 and 4.7 .

The proof of the complex case, namely Theorem 1.8, follows the same lines.

Organization of the paper In Section 2 we introduce our setting and the main tools such as Bergman kernel estimates in normal coordinates. In Section 3 we write the moment $\mathbb{E}\left[\nu_{s}^{k}\right](f)$ as an integral

$$
\int_{\underline{x} \in \mathbb{R} \Sigma^{k}} f(\underline{x}) \mathcal{R}_{d}^{k}(\underline{x})\left|\mathrm{d} \mathrm{V}_{h}\right|^{k} .
$$

This is done by introducing an incidence manifold and using the coarea formula. Far from the diagonal Bergman kernel estimates gives us the asymptotic behaviour of $\mathcal{R}_{d}^{k}$, see Proposition 4.3. The goal of Section 4 is to prove Theorem 1.1. It is a direct consequence of the computation of central moments, Theorem 1.5, which is implied by Theorem 1.4. Theorem 1.4 is proved in this section, admitting a boundedness result, Theorem 4.1, and an asymptotic expansion result, Proposition 4.7.

In Section 5 we prove Theorem 4.1 and Proposition 4.7. This is the core of the paper. Olver multispaces and divided differences coordinates will play a crucial role in the proof of this theorem. In Section 6 we discuss the complex case, giving Theorem 1.8. The proof in the complex case follows the lines of the real one. 


\section{Definitions and main tools}

\subsection{Framework}

In this section we introduce our setting, which is the same as in $[1,10,11]$, but we restrict ourself to the one dimensional case.

- Let $\Sigma$ be a smooth real compact Riemann surface, that is a smooth Riemann surface equipped with an anti-holomorphic involution $c_{\Sigma}$. We denote by $\mathbb{R} \Sigma=\operatorname{Fix}\left(c_{\Sigma}\right)$ its real locus.

- Let $\mathcal{L}$ be a real ample line bundle over $\Sigma$, that is an ample holomorphic line bundle $p: \mathcal{L} \rightarrow \Sigma$ equipped with an anti-holomorphic involution $c_{L}$ such that $p \circ c_{\mathcal{L}}=c_{\Sigma} \circ p$ and $c_{L}$ is complexantilinear in the fibers.

- A real Hermitian metric on $\mathcal{L}$ is a Hermitian metric $h$ such that $c_{\mathcal{L}}^{*} h=\bar{h}_{L}$. We equip $\mathcal{L}$ with a real Hermitian metric $h$ of positive curvature $\frac{i}{2 \pi} \partial \bar{\partial} \phi=\omega \in \Omega^{(1,1)}(\Sigma, \mathbb{R})$. The local potential $\phi$ equals $-\log h\left(e_{\mathcal{L}}, e_{\mathcal{L}}\right)$ where $e_{\mathcal{L}}$ is any local holomorphic trivialization of $\mathcal{L}$.

- The curvature form induces a Kähler metric $\omega(\cdot, i \cdot)$ on $\Sigma$ which restricts to a Riemannian metric over $\mathbb{R} \Sigma$. We denote the Riemannian length form by $\left|\mathrm{dV}_{h}\right|$. We will denote also the Riemannian volume form on $\mathbb{R} \Sigma^{k}$ by $\left|\mathrm{d} V_{h}\right|^{k}$.

- Let $d x=\frac{\omega}{\int_{\Sigma} \omega}$ be the normalized volume form on $\Sigma$.

\subsubsection{The Gaussian measure on $\mathbb{R} H^{0}\left(\Sigma ; \mathcal{L}^{d}\right)$}

We denote by $\mathbb{R} H^{0}(\Sigma ; \mathcal{L})$ the real vector space of real global sections of $\mathcal{L}$, i.e. sections $s \in$ $H^{0}(\Sigma ; \mathcal{L})$ such that $s \circ c_{\Sigma}=c_{\mathcal{L}} \circ s$. The Hermitian metric $h$ induces a Hermitian metric $h^{d}$ on $\mathcal{L}^{d}$ for every integer $d>0$ and also a $L^{2}$-Hermitian product on the space $H^{0}\left(\Sigma ; \mathcal{L}^{d}\right)$ of global holomorphic sections of $\mathcal{L}^{d}$ denoted by $\langle\cdot, \cdot\rangle$ and defined by

$$
\langle\alpha, \beta\rangle=\int_{\Sigma} h^{d}(\alpha, \beta) d x
$$

for any $\alpha, \beta$ in $H^{0}\left(\Sigma ; \mathcal{L}^{d}\right)$.

The $L^{2}$-Hermitian product $\langle\cdot, \cdot\rangle$ on $H^{0}\left(\Sigma ; \mathcal{L}^{d}\right)$ restricts to a $L^{2}$-scalar product on $\mathbb{R} H^{0}\left(\Sigma ; \mathcal{L}^{d}\right)$, also denoted by $\langle\cdot, \cdot\rangle$. Then we have a natural Gaussian measure on $\mathbb{R} H^{0}\left(\Sigma ; \mathcal{L}^{d}\right)$ defined by

$$
\mu(A)=\frac{1}{\sqrt{\pi}^{N_{d}}} \int_{A} e^{-\|s\|^{2}} d s
$$

for any open subset $A \subset \mathbb{R} H^{0}\left(\Sigma ; \mathcal{L}^{d}\right)$ where $d s$ is the Lebesgue measure associated with $\langle\cdot, \cdot\rangle$ and $N_{d}=\operatorname{dim}_{\mathbb{C}} H^{0}\left(\Sigma ; \mathcal{L}^{d}\right)=\operatorname{dim}_{\mathbb{R}} \mathbb{R} H^{0}\left(\Sigma ; \mathcal{L}^{d}\right)$.

\subsection{Bergman kernel}

In this section we recall some asymptotic estimates of the Bergman kernel related to Hermitian line bundles, see $[4,12,18,17]$. Let $(\mathcal{L}, h)$ be a real Hermitian line bundle of positive curvature $\omega$ over a real projective manifold $X$. We denote by $L^{2}\left(X ; \mathcal{L}^{d}\right)$ the space of square integrable global sections of $\mathcal{L}^{d}$ and by $\mathbb{R} L^{2}\left(X ; \mathcal{L}^{d}\right)$ the space of real square integrable global sections. The orthogonal projection from $\mathbb{R} L^{2}\left(X ; \mathcal{L}^{d}\right)$ onto $\mathbb{R} H^{0}\left(X ; \mathcal{L}^{d}\right)$ admits a Schwartz kernel $\mathcal{K}_{d}$. It means that there exists a unique section $\mathcal{K}_{d}$ of the bundle $\mathcal{L}^{d} \otimes\left(\mathcal{L}^{d}\right)^{*}$ over $X \times X$ such that, for any $s \in \mathbb{R} L^{2}\left(X ; \mathcal{L}^{d}\right)$ the projection of $s$ onto $\mathbb{R} H^{0}\left(X ; \mathcal{L}^{d}\right)$ is given by

$$
x \mapsto \int_{y \in X} \mathcal{K}_{d}(x, y)(s(y)) d x .
$$

This Schwartz kernel $\mathcal{K}_{d}(x, y)$ is called the Bergman kernel of $\mathcal{L}^{d}$. 
Remark 2.1. Let $N_{d}$ be the dimension of $\mathbb{R} H^{0}\left(X ; \mathcal{L}^{d}\right)$ and $\left(s_{1}, \ldots, s_{N_{d}}\right)$ be any orthonormal basis of $\mathbb{R} H^{0}\left(X ; \mathcal{L}^{d}\right)$. Then $\mathcal{K}_{d}(x, y)$ is equal to

$$
\sum_{i=1}^{N_{d}} s_{i}(x) \otimes s_{i}(y)^{*}
$$

\subsubsection{Exponential decay}

We recall the following theorem of [18].

Theorem 2.2. Let $(\mathcal{L}, h) \rightarrow X$ be a Hermitian positive line bundle over a complex manifold $X$ of dimension $n$. There exist $C^{\prime}>0$ and $d_{0} \in \mathbb{N}$ such that, for any $m \in \mathbb{N}$, there exists $C_{m}>0$ such that $\forall d \geqslant d_{0}, \forall x, y \in X$

$$
\left\|\mathcal{K}_{d}(x, y)\right\|_{C^{m}} \leqslant C_{m} d^{n+\frac{m}{2}} \exp \left(-C^{\prime} \mathbf{d}_{h}(x, y) \sqrt{d}\right)
$$

where $\mathbf{d}_{h}$ is the geodesic distance in $X$ induced by $h$ and the norms of the derivates of $K_{d}$ are also induced by $h$.

\subsubsection{Normal coordinates}

In this section we define our preferred coordinates and trivializations to which we refer to as normal coordinates. For a more detailed introduction of these coordinates, see [16, Section 3.1].

Let $(\mathcal{L}, h)$ be a real Hermitian line bundle of positive curvature $\omega$ over a projective manifold $X$. Let $x$ be a point in $X$ and $U$ be a small neighborhood of $x$. The normal coordinates around $x$ is the exponential coordinates $\exp _{x}: z \in V \subset T_{x} X \mapsto U \subset X$ (which, in general, is not holomorphic) together with the local trivialization $\mathcal{L}_{x}^{d} \times\left. U \simeq \mathcal{L}^{d}\right|_{U}$ of $\mathcal{L}^{d}$ given, at any point $\exp _{x}(z) \in U$, by the parallel transport (induced by the Chern connection of $\mathcal{L}^{d}$ ) of the fiber $\mathcal{L}_{x}^{d}$ along the geodesic $t \mapsto \exp _{x}(t z)$. For any $d>0$, the scaled normal coordinates around $x$ is the composition of the map $T \mapsto z=\frac{T}{\sqrt{d}}$ with the normal coordinate around $x$.

In the presence of a real structure $c_{X}$, for any $x \in \mathbb{R} X$, the restriction of the exponential map $\left.\exp _{x}\right|_{T_{x} \mathbb{R} \Sigma}$ coincides with the exponential map associated with the Riemannian metric $\mathbb{R} X$ induced by $\omega$. The normal coordinates around a real point $x \in \mathbb{R} X$ is the (real) exponential coordinates $\exp _{x}: z \in V \subset T_{x} \mathbb{R} X \mapsto U \subset \mathbb{R} X$ together with the local trivialization $\mathbb{R}_{x}^{d} \times\left. U \simeq \mathbb{R}^{d}\right|_{U}$ of $\mathbb{R} \mathcal{L}^{d}$ around $x$ given, at any point $\exp _{x}(z) \in U$, by the parallel transport of the fiber $\mathbb{R} \mathcal{L}_{x}^{d}$ along the geodesic $t \mapsto \exp _{x}(t z)$.

\subsubsection{Near diagonal estimate}

The following theorem says that in the (scaled) normal coordinates $U_{x}$ around a point $x \in X$ the geometry of $\mathcal{L}_{\mid U_{x}}^{d} \rightarrow U_{x}$ looks like the geometry of the Bargmann-Fock space (see Section 5.4.2), at least in a ball of size $B\left(x ; \frac{R \log d}{\sqrt{d}}\right)$ for large $d$ and any fixed $R>0$. The following theorem is the main theorem of [7] (see also [17, Theorem 4.18], [12, 4]).

Theorem 2.3. Let $(\mathcal{L}, h) \rightarrow X$ be a Hermitian positive line bundle over a complex manifold $X$ of dimension $n$. Fix $m \in \mathbb{N}$ and $R>0$. Then, for any $\alpha \in(0,1)$ any $x \in X$ and any $z, w \in B\left(x, R \frac{\log d}{\sqrt{d}}\right)$, one have

$$
\left\|\mathcal{K}_{d}(z, w)-\left(\frac{d}{\pi}\right)^{n} e^{\frac{-a\|z-w\|^{2}}{2}}\right\|_{C^{m}}=O\left(d^{n-\alpha}\right)
$$

in the normal coordinates around $x$. Here, $\|\cdot\|$ is the norm on $T_{x} X$ induced by $h$ and $\|\cdot\|_{C^{m}}$ is the $C^{m}$-norm on $\left(\mathcal{L}^{d}\right)_{x}$ induced by $h$. The error term does not depend on $x, z, w$ but only on $m, R$ and $\alpha$.

If $X$ is a real algebraic variety, we obtain:

Theorem 2.4. Let $(\mathcal{L}, h) \rightarrow X$ be a real Hermitian positive line bundle over a real algebraic variety $X$ of dimension $n$. Fix $m \in \mathbb{N}$ and $R>0$. For any $\alpha \in(0,1)$, any $x \in \mathbb{R} X$ and any $z, w \in B\left(x, R \frac{\log d}{\sqrt{d}}\right)$, one have 


$$
\left\|\mathcal{K}_{d}(z, w)-\left(\frac{d}{\pi}\right)^{n} e^{\frac{-d\|z-w\|^{2}}{2}}\right\|_{C^{m}}=O\left(d^{n-\alpha}\right)
$$

in the normal coordinates around $x$. Here, $\|\cdot\|_{C^{m}}$ is the $C^{m}$-norm on $\left(\mathcal{L}^{d}\right)_{x}$ induced by $h$. The error term does not depend on $x, z, w$ but only on $m, R$, and $\alpha$.

Proof. This is Theorem 2.3, restricting everything to the real locus $\mathbb{R} X$ of $X$.

\subsubsection{Scaled Bergman kernel}

These theorems suggest us the following

Definition 2.5. Fix a real point $x \in \mathbb{R} X$. We define the scaled Bergman kernel by $K_{d}(Z, W)=$ $\frac{1}{d^{n}} \mathcal{K}_{d}\left(\frac{Z}{\sqrt{d}}, \frac{W}{\sqrt{d}}\right)$, where $Z, W$ are the scaled (real) normal coordinates around $x$ (that are $Z=\sqrt{d} z$ and $W=\sqrt{d} w)$ and the local Bergman kernel by $K_{\mathbb{C}^{n}}(Z, W)=\frac{1}{\pi^{n}} e^{\frac{-\|Z-W\|^{2}}{2}}$.

In particular, Theorem 2.4 can be written as:

Theorem 2.6. Fix $k \in \mathbb{N}$ and $R>0$. For any $\alpha \in(0,1)$, for any $x \in \mathbb{R} X$ and any $Z, W \in$ $B(x, R \log d)$ we have

$$
\left\|K_{d}(Z, W)-K_{\mathbb{C}^{n}}(Z, W)\right\|_{C^{m}}=O\left(\frac{1}{d^{\alpha}}\right)
$$

in the scaled normal coordinates around $x$. Moreover the error term only depends on $m, R$ and $\alpha$.

\section{Incidence manifold and density function}

Throughout this section, we will denote by $(\mathcal{L}, h)$ a real ample Hermitian line bundle over a real Riemann surface $\Sigma$, see Section 2.1.

In the first susbection we define some modified measures $\tilde{\nu}_{s}^{k}$. Then, we introduce an incidence manifold and use the coarea formula to write the modified moments $\mathbb{E}\left[\tilde{\nu}_{s}^{k}\right]$ as an integral of a density function $\mathcal{R}_{d}^{k}$ over $\mathbb{R} \Sigma^{k}$. This is done in the second subsection. The estimate of the density function $\mathcal{R}_{d}^{k}$ is the most important step in Theorem 1.4. We start the study of $\mathcal{R}_{d}^{k}$ by writing it as a fraction (Proposition 3.9 ) and by giving an off-diagonal estimate (Proposition 4.3).

\subsection{Zeros of random real sections}

Let $s \in \mathbb{R} H^{0}\left(\Sigma ; \mathcal{L}^{d}\right)$ be any real section of $\mathcal{L}^{d}$, that is any global holomorphic section of $\mathcal{L}^{d}$ such that $s \circ c_{\Sigma}=c_{\mathcal{L}} \circ s$, where $c_{\mathcal{L}}$ and $c_{\Sigma}$ are the real structures of $\mathcal{L}$ and $\Sigma$, see Section 2.1.

Definition 3.1. - To any non-zero section $s \in \mathbb{R} H^{0}\left(\Sigma ; \mathcal{L}^{d}\right)$ we associate the following empirical measure $\nu_{s}=\sum_{x \in Z_{s}} \delta_{x}$ where $Z_{s}=\mathbb{R} \Sigma \cap\{s=0\}$ is the real vanishing locus of $s$ and $\delta_{x}$ is the Dirac measure at $x \in Z_{s}$. It induces an empirical measure $\nu_{s}^{k}$ on $\mathbb{R} \Sigma^{k}$ for any $k \in \mathbb{N}^{*}$, defined, for any $f \in C^{0}\left(\mathbb{R} \Sigma^{k}\right)$, by

$$
\nu_{s}^{k}(f)=\sum_{\left(x_{1}, \ldots, x_{k}\right) \in Z_{s}^{k}} f\left(x_{1}, \ldots, x_{k}\right)
$$

- For any $s \in \mathbb{R} H^{0}\left(\Sigma ; \mathcal{L}^{d}\right)$ and any $k \in \mathbb{N}$ we define $\tilde{\nu}_{s}^{k}$ to be the following modified empirical measure:

$$
\tilde{\nu}_{s}^{k}(f)=\sum_{\substack{\left(x_{1}, \ldots, x_{k}\right) \in Z_{s}^{k} \\ x_{i} \neq x_{j}}} f\left(x_{1}, \ldots, x_{k}\right) .
$$

\subsubsection{Partitions of $k$ elements}

Let $\mathcal{P}_{k}$ be the set of all the partitions of $\{1, \ldots, k\}$, that means the set of all

$$
I=\left\{\left\{1_{1}, \ldots, 1_{k_{1}}\right\}, \ldots,\left\{m_{1}, \ldots, m_{k_{m}}\right\}\right\}
$$


such that $\left\{1_{1}, \ldots, 1_{k_{1}}\right\} \sqcup \cdots \sqcup\left\{m_{1}, \ldots, m_{k_{m}}\right\}=\{1, \ldots, k\}$. For any $I \in \mathcal{P}_{k}$, we write $\left(x_{1}, \ldots, x_{k}\right) \in$ $Z_{s, I}^{k}$ if and only if $\left(x_{1}, \ldots, x_{k}\right) \in Z_{s}^{k}$ and $x_{i_{l}}=x_{i_{h}}$ for any $l, h \in\left\{1, \ldots, k_{i}\right\}$ and $x_{i_{l}} \neq x_{j_{h}}$ if $i \neq j \in\{1, \ldots, m\}$. We then write

$$
\nu_{s}^{k}(f)=\sum_{I \in \mathcal{P}_{k}} \sum_{\left(x_{1}, \ldots, x_{k}\right) \in Z_{s, I}^{k}} f\left(x_{1}, \ldots, x_{k}\right) .
$$

Now, we denote by $\widetilde{\mathcal{P}}_{k}=\mathcal{P}_{k} \backslash\{\{1\},\{2\}, \ldots,\{k\}\}$ so that

$$
\nu_{s}^{k}(f)=\sum_{I \in \tilde{\mathcal{P}}_{k}} \sum_{\left(x_{1}, \ldots, x_{k}\right) \in Z_{s, I}^{k}} f\left(x_{1}, \ldots, x_{k}\right)+\tilde{\nu}_{s}^{k}(f) .
$$

For any partition $I=\left\{\left\{1_{1}, \ldots, 1_{k_{1}}\right\}, \ldots,\left\{m_{1}, \ldots, m_{k_{m}}\right\}\right\} \in \widetilde{\mathcal{P}}_{k}$, we denote by $j_{I}: \mathbb{R} \Sigma^{m} \rightarrow \mathbb{R} \Sigma^{k}$ the inclusion defined by $\left(x_{1}, \ldots, x_{m}\right) \mapsto\left(\tilde{x}_{1}, \ldots, \tilde{x}_{k}\right)$ where, for any $i, \tilde{x}_{i_{j}}=x_{i}$ for any $j=1, \ldots, k_{j}$. The following proposition is a direct consequence of the definitions just given.

Proposition 3.2. For every real section $s \in \mathbb{R} H^{0}\left(\Sigma ; \mathcal{L}^{d}\right)$, every integer $k \in \mathbb{N}$ and every partition $I=\left\{\left\{1_{1}, \ldots, 1_{k_{1}}\right\}, \ldots,\left\{m_{1}, \ldots, m_{k_{m}}\right\}\right\} \in \mathcal{P}_{k}$, we have

$$
\sum_{\left(x_{1}, \ldots, x_{k}\right) \in Z_{s, I}^{k}} f\left(x_{1}, \ldots, x_{k}\right)=\tilde{\nu}_{s}^{m}\left(j_{I}^{*} f\right)
$$

so that

$$
\nu_{s}^{k}(f)=\tilde{\nu}_{s}^{k}(f)+\sum_{I \in \widetilde{\mathcal{P}}_{k}} \tilde{\nu}_{s}^{m_{I}}\left(j_{I}^{*} f\right)
$$

\subsubsection{Modified moments}

To understand $\nu_{s}^{k}(f)$, it is enough, in fact equivalent, to understand $\tilde{\nu}_{s}^{k}(f)$. We will study the expectation of this new random variable, namely

$$
\mathbb{E}\left[\tilde{\nu}_{s}^{k}\right](f)=\int_{s \in \mathbb{R} H^{0}\left(\Sigma ; \mathcal{L}^{d}\right)} \sum_{\substack{\left(x_{1}, \ldots, x_{k}\right) \in Z_{s}^{k} \\ x_{i} \neq x_{j}}} f\left(x_{1}, \ldots, x_{k}\right) d \mu(s)
$$

We call $\mathbb{E}\left[\tilde{\nu}_{s}^{k}\right]$ the modified $k$-moment of $\nu_{s}$.

\subsection{Incidence manifold and density function $\mathcal{R}_{d}^{k}$}

We introduce an incidence manifold $\mathcal{I}$ that comes equipped with two projections respectively to $\mathbb{R} H^{0}\left(\Sigma ; \mathcal{L}^{d}\right)$ and to $\mathbb{R} \Sigma^{k}$. Following [22] (see also [11], [1]) we will apply the coarea formula (see [9, Lemma 3.2.3] or [22, Theorem 1]) to these two projections to write the modified moment $\mathbb{E}\left[\tilde{\nu}_{s}^{k}\right]$ as an integral over $\mathbb{R} \Sigma^{k}$. The resulting formula is often known as Kac-Rice formula, see for example [3, Theorem 3.2].

\subsubsection{Incidence manifold}

Let $\Delta$ be the diagonal of $\mathbb{R} \Sigma^{k}$ defined by

$$
\Delta=\left\{\left(x_{1}, \ldots, x_{k}\right) \in \mathbb{R} \Sigma^{k} \mid \exists i \neq j, x_{i}=x_{j}\right\}
$$

and set

$$
\mathcal{I}=\left\{\left(s, x_{1}, \ldots, x_{k}\right) \in \mathbb{R} H^{0}\left(\Sigma ; \mathcal{L}^{d}\right) \times\left(\mathbb{R} \Sigma^{k} \backslash \Delta\right) \mid s\left(x_{i}\right)=0 \quad i=1, \ldots, k\right\} .
$$

We denote by $\pi_{\Sigma}\left(\right.$ resp. $\left.\pi_{H}\right)$ the projection $\mathcal{I} \rightarrow \mathbb{R} \Sigma^{k}\left(\right.$ resp. $\mathcal{I} \rightarrow \mathbb{R} H^{0}\left(\Sigma ; \mathcal{L}^{d}\right)$ ).

Proposition 3.3. Let $\mathcal{L}$ be a positive real line bundle over a real Riemann surface $\Sigma$. Then, for large $d \in \mathbb{N}$, the set $\mathcal{I}$ is a smooth manifold. We call $\mathcal{I}$ the incidence manifold.

Proof. Consider the map $\mathbb{R} H^{0}\left(\Sigma ; \mathcal{L}^{d}\right) \times\left(\mathbb{R} \Sigma^{k} \backslash \Delta\right) \rightarrow \mathbb{R} \mathcal{L}^{d} \times \cdots \times \mathbb{R} \mathcal{L}^{d}$ defined by $\left(s, x_{1}, \ldots, x_{k}\right) \mapsto$ $\left(s\left(x_{1}\right), \ldots, s\left(x_{k}\right)\right)$. We have to prove that 0 is a regular value. The derivative of this map is

$$
\left(\dot{s}, \dot{x}_{1}, \ldots, \dot{x}_{k}\right) \mapsto\left(\dot{s}\left(x_{1}\right)+\nabla_{\dot{x}_{1}} s\left(x_{1}\right), \ldots, \dot{s}\left(x_{k}\right)+\nabla_{\dot{x}_{k}} s\left(x_{k}\right)\right) .
$$

By the positivity of $\mathcal{L}$ and by Riemann-Roch theorem, there exists $d_{0}$ such that for any $d \geqslant d_{0}$ and any $\left(x_{1}, \ldots, x_{k}\right) \in \mathbb{R} \Sigma^{k} \backslash \Delta$ we can find sections $\dot{s}_{1}, \ldots, \dot{s}_{k}$ such that $\dot{s}_{i}\left(x_{i}\right) \neq 0$ and $\dot{s}_{i}\left(x_{j}\right)=0$ for $i \neq j$. This implies that 0 is a regular value. 


\subsubsection{The density function $\mathcal{R}_{d}^{k}$}

Definition 3.4. The normal jacobian $\operatorname{Jac}_{N} u$ of a submersion $u: M \rightarrow N$ between Riemannian manifolds is the determinant of the differential of the map restricted to the orthogonal of its kernel. Equivalently, if $d u_{p}$ is the differential of $u$ at $p$, then the normal jacobian is equal to $\sqrt{\operatorname{det}\left(d u_{p} d u_{p}^{*}\right)}$, where $d u_{p}^{*}$ is the adjoint of $d u_{p}$ with respect to the scalar products on $T_{p} M$ and $T_{u(p)} N$. 3.1.

Recall that we have defined the modified moment to be $\mathbb{E}\left[\tilde{\nu}_{s}^{k}\right]$, where $\tilde{\nu}_{s}^{k}$ was defined in Definition

Proposition 3.5. Let $(\mathcal{L}, h)$ be a real Hermitian line bundle over a real Riemann surface $\Sigma$. Then, for large $d \in \mathbb{N}$, we have

$$
\mathbb{E}\left[\tilde{\nu}_{s}^{k}\right](f)=\int_{\underline{x} \in \mathbb{R} \Sigma^{k} \backslash \Delta} f(\underline{x}) \mathcal{R}_{d}^{k}(\underline{x})\left|d V_{h}\right|^{k}
$$

where $\tilde{\nu}_{s}^{k}$ was defined in Definition 3.1 and

$$
\mathcal{R}_{d}^{k}(\underline{x})=\int_{\pi_{\Sigma}^{-1}(\underline{x})} \frac{1}{\left|\operatorname{Jac}_{N}\left(\pi_{\Sigma}\right)\right|} d \mu_{\mid \pi_{\Sigma}^{-1}(\underline{x})} .
$$

Proof. We consider

$$
\mathbb{E}\left[\tilde{\nu}_{s}^{k}\right](f)=\int_{s \in \mathbb{R} H^{0}\left(\Sigma ; \mathcal{L}^{d}\right)} \sum_{\substack{\left(x_{1}, \ldots, x_{k}\right) \in Z_{s}^{k} \\ x_{i} \neq x_{j}}} f\left(x_{1}, \ldots, x_{k}\right) d \mu(s) .
$$

Using $\pi_{H}$ we pull-back the integral over $\mathcal{I}$. On $\mathcal{I}$ we put the (singular) metric $\pi_{H}^{*}\langle\cdot, \cdot\rangle$. We have $\mathbb{E}\left[\tilde{\nu}_{s}^{k}\right](f)=\int_{\mathcal{I}}\left(\pi_{\Sigma}^{*} f\right)\left(s, x_{1}, \ldots, x_{k}\right)\left(\pi_{H}^{*} d \mu\right)\left(s, x_{1}, \ldots, x_{k}\right)$. The coarea formula (see [9, Lemma 3.2.3] or [22, Theorem 1]) applied to the submersion $\pi_{\Sigma}: \mathcal{I} \rightarrow \mathbb{R} \Sigma^{k}$ gives us the result.

Definition 3.6. We call density function the function $\mathcal{R}_{d}^{k}: \mathbb{R} \Sigma^{k} \backslash \Delta \rightarrow \mathbb{R}$ that appeared in Proposition 3.5 .

Proposition 3.7. For any permutations $\sigma \in \mathcal{S}_{k}$ we have $\mathcal{R}_{d}^{k} \circ \sigma=\mathcal{R}_{d}^{k}$.

Proof. The symmetric group $\mathcal{S}_{k}$ acts by isometries on $\mathbb{R} \Sigma^{k}$ and on $\mathcal{I}$, this implies the result.

Definition 3.8. For $\underline{x}=\left(x_{1}, \ldots, x_{k}\right) \in \mathbb{R} \Sigma^{k} \backslash \Delta$ we denote by $\mathbb{R} H_{\underline{x}}^{0}=\mathbb{R} H_{x_{1}, \ldots, x_{k}}^{0}$ the subspace of real global sections $s \in \mathbb{R} H^{0}\left(\Sigma ; \mathcal{L}^{d}\right)$ such that $s\left(x_{i}\right)=0$ for any $i \in\{1, \ldots, k\}$, that is the kernel of the evaluation map

$$
e v_{\underline{x}}: \mathbb{R} H^{0}\left(\Sigma ; \mathcal{L}^{d}\right) \rightarrow\left(\mathbb{R} \mathcal{L}^{d}\right)_{x_{1}} \times \cdots \times\left(\mathbb{R} \mathcal{L}^{d}\right)_{x_{k}}
$$

defined by $s \mapsto\left(s\left(x_{1}\right), \ldots, s\left(x_{k}\right)\right)$.

Proposition 3.9. Let $\mathcal{R}_{d}^{k}$ be the density function defined in Proposition 3.5. Then, for any $\underline{x}=$ $\left(x_{1}, \ldots, x_{k}\right) \in \mathbb{R} \Sigma^{k} \backslash \Delta$, we have

$$
\mathcal{R}_{d}(\underline{x})=\frac{\mathcal{N}_{d}^{k}(\underline{x})}{\mathcal{D}_{d}^{k}(\underline{x})}
$$

where

$$
\mathcal{N}_{d}^{k}(\underline{x})=\int_{s \in \mathbb{R} H_{\underline{x}}^{0}}\left\|\nabla s\left(x_{1}\right)\right\| \cdots\left\|\nabla s\left(x_{k}\right)\right\| d \mu_{\mid \mathbb{R} H_{\underline{x}}^{0}}(s)
$$

and

$$
\mathcal{D}_{d}^{k}(\underline{x})=\left|\operatorname{Jac}_{N}\left(e v_{\underline{x}}\right)\right| .
$$

Here we have denoted by $\|\cdot\|$ the norms induced by the Hermitian metric $h$.

Proof. This is the Kac-Rice formula (see [3, Theorem 3.2]). Let us sketch the proof.

Fix $\underline{x}=\left(x_{1}, \ldots, x_{k}\right) \in \mathbb{R} \Sigma^{k} \backslash \Delta$, by Proposition 3.5 we have

$$
\mathcal{R}_{d}^{k}(\underline{x})=\int_{\pi_{\Sigma}^{-1}(\underline{x})} \frac{1}{\left|\operatorname{Jac}_{N}\left(\pi_{\Sigma}\right)\right|} d \mu_{\mid \pi_{\Sigma}^{-1}(\underline{x})^{\prime}} .
$$


As $\pi_{H}$ is (almost everywhere) a local isometry, we can pushforward this integral onto $\mathbb{R} H^{0}\left(\Sigma ; \mathcal{L}^{d}\right)$ and we obtain

$$
\mathcal{R}_{d}^{k}(\underline{x})=\int_{\mathbb{R} H_{\underline{x}}^{0}} \frac{1}{\left|\left(\pi_{H}^{-1}\right)^{*} J a c_{N}\left(\pi_{\Sigma}\right)\right|} d \mu_{\mid \mathbb{R} H_{\underline{x}}^{0}}
$$

Let $\left(s, x_{1}, \ldots, x_{k}\right)$ be a point in $\pi_{\Sigma}^{-1}\left(x_{1}, \ldots, x_{k}\right) \subset \mathcal{I}$ such that $\nabla s\left(x_{i}\right)$ is invertible for any $i \in$ $\{1, \ldots, k\}$ and let $\left(\dot{s}, \dot{x}_{1}, \ldots, \dot{x}_{k}\right)$ be any tangent vector of $\left(s, x_{1}, \ldots, x_{k}\right)$. We have $d \pi_{\Sigma} \cdot\left(\dot{s}, \dot{x}_{1}, \ldots, \dot{x}_{k}\right)=$ $\left(\dot{x}_{1}, \ldots, \dot{x}_{k}\right)$. Remember that, by definition, the norm $\left\|\left(\dot{s}, \dot{x}_{1}, \ldots, \dot{x}_{k}\right)\right\|$ on $\mathcal{I}$ equals $\|\dot{s}\|_{L^{2}}$ and also that for every $i=1, \ldots, k$ we have $\dot{s}\left(x_{i}\right)+\nabla_{\dot{x}_{i}} s\left(x_{i}\right)=0$, so that

$$
d \pi_{\Sigma} \cdot\left(\dot{s}, \dot{x}_{1}, \ldots, \dot{x}_{k}\right)=\left(\dot{x}_{1}, \ldots, \dot{x}_{k}\right)=\left(-\nabla s\left(x_{1}\right)^{-1} \circ \dot{s}\left(x_{1}\right), \ldots,-\nabla s\left(x_{k}\right)^{-1} \circ \dot{s}\left(x_{k}\right)\right) .
$$

Consider the map

$$
B: T_{x_{1}} \mathbb{R} \Sigma \times \cdots \times T_{x_{k}} \mathbb{R} \Sigma \rightarrow\left(\mathbb{R} \mathcal{L}^{d}\right)_{x_{1}} \times \cdots \times\left(\mathbb{R} \mathcal{L}^{d}\right)_{x_{k}}
$$

defined by $B\left(\dot{x}_{1}, \ldots, \dot{x}_{k}\right)=\left(\nabla_{\dot{x}_{1}} s\left(x_{1}\right), \ldots, \nabla_{\dot{x}_{k}} s\left(x_{k}\right)\right)$. We remark that $B$ is a diagonal map so that $\mathrm{Jac} B=\left\|\nabla s\left(x_{1}\right)\right\| \cdots\left\|\nabla s\left(x_{k}\right)\right\|$. We have

$$
d \pi_{\Sigma}=B^{-1} \circ e v_{x_{1}, \ldots, x_{k}} \circ d \pi_{H}
$$

and this implies

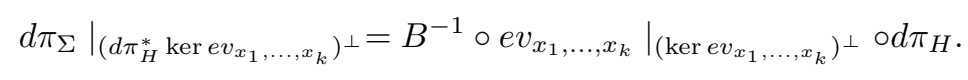

It follows that

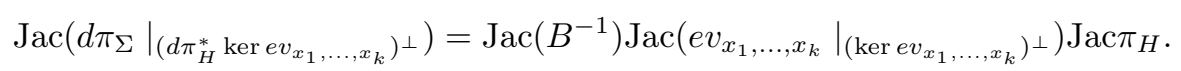

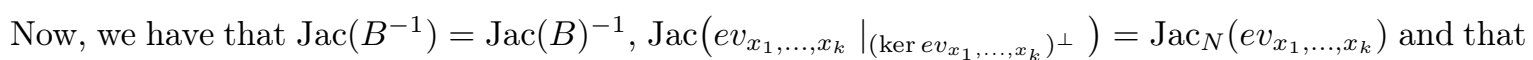
the normal Jacobian of $\pi_{H}$ is equal to 1 as $\pi_{H}$ is a local isometry. We conclude by integrating over $\mathbb{R} H_{\underline{x}}^{0}$ and observing that the set of $s \in \mathbb{R} H_{\underline{x}}^{0}$ such that $\nabla s\left(x_{i}\right)=0$ for some $i \in\{1, \ldots, k\}$ has measure zero.

Proposition 3.10. Let $\mathcal{L}$ be a positive real Hermitian line bundle over a real Riemann surface $\Sigma$ and $\underline{x}=\left(x_{1}, \ldots, x_{k}\right)$ be a point in $\mathbb{R} \Sigma^{k} \backslash \Delta$. Then

$$
\operatorname{Jac}_{N}\left(e v_{\underline{x}}\right)=\sqrt{\operatorname{det}\left(\left\|\mathcal{K}_{d}\left(x_{i}, x_{j}\right)\right\|\right)_{(i, j)}}
$$

where $e v_{\underline{x}}$ is the evaluation map defined in Definition 3.8.

Proof. We fix an orthonormal basis $\left\{s_{1}, \ldots, s_{N_{d}}\right\}$ of $\mathbb{R} H^{0}\left(\Sigma ; \mathcal{L}^{d}\right)$. The Bergman kernel of the orthogonal projection onto $\mathbb{R} H^{0}\left(\Sigma ; \mathcal{L}^{d}\right)$ is

$$
\mathcal{K}_{d}(x, y)=\sum_{i=1}^{N_{d}} s_{i}(x) \otimes s_{i}(y)^{*} .
$$

We also fix a unit vector $e_{i}$ over each fiber $\mathbb{R} \mathcal{L}_{x_{i}}^{d}, i \in\{1, \ldots, k\}$. The matrix associated with $e v_{\underline{x}}$ with respect to these basis equals $M=\left(h^{d}\left(s_{l}\left(x_{i}\right), e_{i}\right)\right)_{i, l}$. Now, the matrix $M M^{*}$ is exactly the symmetric matrix $\left(\left\|\mathcal{K}_{d}\left(x_{i}, x_{j}\right)\right\|\right)_{i, j}$. We conclude by taking the square root of the determinant of $M M^{*}$, which is exactly $\operatorname{Jac}_{N}\left(e v_{\underline{x}}\right)$.

Proposition 3.11. Let $\mathcal{L}$ be a positive real Hermitian line bundle over a real Riemann surface $\Sigma$. Then there exists an integer $d_{\mathcal{L}} \in \mathbb{N}$ such that for any $\left(x_{1}, \ldots, x_{k}\right) \in \mathbb{R} \Sigma^{k} \backslash \Delta$ and $d \geqslant d_{\mathcal{L}}$ the map

$$
e v_{\underline{x}}: \mathbb{R} H^{0}\left(\Sigma ; \mathcal{L}^{d}\right) \rightarrow \mathbb{R} \mathcal{L}_{x_{1}}^{d} \times \cdots \times \mathbb{R} \mathcal{L}_{x_{k}}^{d}
$$

defined by $s \mapsto\left(s\left(x_{1}\right), \ldots, s\left(x_{k}\right)\right)$ is surjective.

Proof. Since $\mathcal{L}$ is positive, there exists $d_{\mathcal{L}}$ such that for $d \geq d_{\mathcal{L}}$ and for every point $\left(x_{1}, \ldots, x_{k}\right) \in$ $\mathbb{R} \Sigma^{k} \backslash \Delta$, we have $H^{1}\left(\Sigma ; \mathcal{L}^{d}\left(-\sum_{j=1}^{k} x_{j}\right)\right)=0$. From the Riemann-Roch theorem we then know that for $i \in\{1, \ldots, k\}$

$$
\operatorname{dim} H^{0}\left(\Sigma ; \mathcal{L}^{d}\left(-\sum_{j=1}^{k} x_{j}\right)\right)=\operatorname{dim} H^{0}\left(\Sigma ; \mathcal{L}^{d}\left(-\sum_{j \neq i} x_{j}\right)\right)-1 .
$$

This proves that there are sections $s_{i}$ such that $s_{i}\left(x_{i}\right) \neq 0$ and $s_{i}\left(x_{j}\right)=0$ for $i \in\{1, \ldots, k\}$. The surjectivity of $e v_{\underline{x}}$ follows. 
Corollary 3.12. There exists a positive integer $d_{0} \in \mathbb{N}$ such that for any $d \geqslant d_{0}$ and for any $\left(x_{1}, \ldots, x_{k}\right) \in \mathbb{R} \Sigma^{k} \backslash \Delta$,

$$
\mathcal{D}_{d}^{k}\left(x_{1}, \ldots, x_{k}\right)>0 \text {. }
$$

Proof. From Proposition 3.9 we have $\mathcal{D}_{d}^{k}(\underline{x})=\operatorname{Jac}_{N} e v_{\underline{x}}$. The result follows from Proposition 3.11 and from the fact that the normal Jacobian of a map is positive if and only if the map is surjective.

Remark 3.13. For a point $\left(x_{1}, \ldots, x_{k}\right)$ of the diagonal $\Delta \subset \mathbb{R} \Sigma^{k}$ the map $e v_{x_{1}, \ldots, x_{k}}$ is not surjective, so that $\mathcal{D}_{d}^{k}=0$ on the diagonal.

\section{Proof of the main theorems}

This section is devoted to the proof of Theorem 1.1. For this purpose, we use Markov inequality together with the computations of the central moments of $\# Z_{s}$ given by Theorem 1.5. Theorem 1.5 is itself a consequence of Theorem 1.4, which computes all the moments of $\# Z_{s}$. For this, we strongly use a uniform bound on the $L^{\infty}$-norm of the density function $\mathcal{R}_{d}^{k}$. This uniform bound is given by Theorem 4.1, that we prove in Sections 5.1-5.5.

\subsection{Uniform bound on the $L^{\infty}$-norm of the density function $\mathcal{R}_{d}^{k}$}

The main result of this section is the following uniform boundedness result for the density function defined in Proposition 3.5. We prove this result in Section 5.

Theorem 4.1. Let $(\mathcal{L}, h)$ be a positive real Hermitian line bundle over a real Riemann surface $\Sigma$. Then, there exists a constant $C$ and an integer $d_{k} \in \mathbb{N}$ such that for any $d \geq d_{k}$ and $\underline{x}=\left(x_{1}, \ldots, x_{k}\right) \in$ $\mathbb{R} \Sigma^{k}$

$$
\frac{1}{\sqrt{d}^{k}} \mathcal{R}_{d}^{k}(\underline{x}) \leq C
$$

We denote by $\mathbf{d}_{h}$ the distance on $\Sigma$ induced by the curvature form $\omega$ of $(\mathcal{L}, h)$. The following result is a consequence of the exponential decay of the Bergman kernel.

Proposition 4.2. Let $m, s \in \mathbb{N}$ be such that $m+s=k$. Let $\left(x_{1}, \ldots, x_{m}, y_{1}, \ldots, y_{s}\right) \in \mathbb{R} \Sigma^{k}$ be such that $\mathbf{d}_{h}\left(x_{i}, y_{j}\right)>\frac{\log d}{C^{\prime} \sqrt{d}}$, where $C^{\prime}$ is the constant appearing in Theorem 2.2. Then, we have

$$
\frac{1}{\sqrt{d}^{k}} \mathcal{R}_{d}^{k}\left(x_{1}, \ldots, x_{l}, y_{1}, \ldots, y_{s}\right)=\frac{1}{\sqrt{d}^{l}} \mathcal{R}_{d}^{l}\left(x_{1}, \ldots, x_{l}\right) \frac{1}{\sqrt{d}^{s}} \mathcal{R}_{d}^{s}\left(y_{1}, \ldots, y_{s}\right)+O\left(\frac{1}{d}\right) .
$$

Proof. For clarity of exposition, we prove this lemma for $l=s=1$. The general case follows the same lines. Let $x, y \in \mathbb{R} \Sigma^{2}$ be two points such that $\mathbf{d}_{h}(x, y)>\frac{\log d}{C^{\prime} \sqrt{d}}$. The density function write $\mathcal{R}_{d}^{2}=\frac{\mathcal{N}_{d}^{2}}{\mathcal{D}_{d}^{2}}$, see Proposition 3.9. We treat separately the denominator $\mathcal{D}_{d}^{2}$ and the numerator $\mathcal{N}_{d}^{2}$.

By Theorem 2.2, we have $\frac{1}{d}\left\|\mathcal{K}_{d}\left(x_{i}, x_{j}\right)\right\|=O\left(\frac{1}{d}\right)$. By Propositions 3.9 and 3.10 we then obtain

$$
\begin{gathered}
\frac{1}{d} \mathcal{D}_{d}^{k}(x, y)=\frac{1}{d} \sqrt{\operatorname{det}\left[\begin{array}{cc}
\left\|\mathcal{K}_{d}(x, x)\right\| & \left\|\mathcal{K}_{d}(x, y)\right\| \\
\left\|\mathcal{K}_{d}(y, x)\right\| & \left\|\mathcal{K}_{d}(y, y)\right\|
\end{array}\right]}= \\
=\frac{1}{d} \sqrt{\left\|\mathcal{K}_{d}(x, x)\right\|} \sqrt{\left\|\mathcal{K}_{d}(y, y)\right\|}+O\left(\frac{1}{d}\right)=\frac{1}{\sqrt{d}} \mathcal{D}_{d}^{1}(x) \frac{1}{\sqrt{d}} \mathcal{D}_{d}^{1}(y)+O\left(\frac{1}{d}\right) .
\end{gathered}
$$

We now study the numerator. Let $\mathbb{R} H_{x}^{0}=\left\{s \in \mathbb{R} H^{0}\left(\Sigma ; \mathcal{L}^{d}\right), s(x)=0\right\}$ be the kernel of the evaluation map at $x$ and $j_{x}^{1}: s \in \mathbb{R} H_{x}^{0} \mapsto \nabla s(x)$ be the 1-jet evaluation map. The integrand of the numerator depends only on the 1 -jets of sections $s$ at the points $x, y$ so that, after an integration over $\operatorname{ker} j_{x}^{1} \cap \operatorname{ker} j_{y}^{1}$, we have

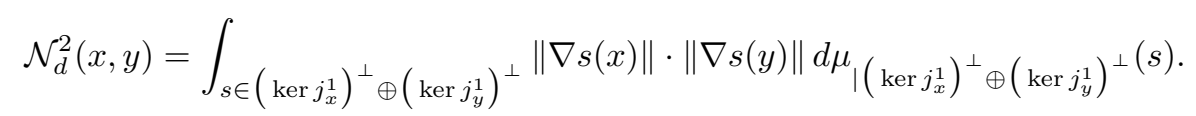

Let $s_{x}$ (resp. $s_{y}$ ) be a unit norm section generating the orthogonal of ker $j_{x}^{1}$ (resp. $\operatorname{ker} j_{y}^{1}$ ). We can then write every $s \in\left(\operatorname{ker} j_{x}^{1}\right)^{\perp} \oplus\left(\operatorname{ker} j_{y}^{1}\right)^{\perp}$ as $s=a s_{x}+b s_{y}$. As the distance between the points $x$ and $x$ is bigger than $\frac{\log d}{C^{\prime} \sqrt{d}}$, a result of Tian (see [23], Lemma 3.1) implies that the sections $s_{x}$ and 
$s_{y}$ are asymptotically orthogonal, more precisely $\left\langle s_{x} ; s_{y}\right\rangle_{L^{2}}=O\left(\frac{1}{d}\right)$. Moreover the pointwise norm of $\frac{1}{d} \nabla s_{x}$ at $y$ is $O\left(\frac{1}{d}\right)$ (and the same holds for $\frac{1}{d} \nabla s_{y}$ at $x$ ). These sections are called peak sections, see for example $[1,11,23]$. For these reasons we have that

$$
\begin{gathered}
\frac{1}{d^{2}} \mathcal{N}_{d}^{2}(x, y)=\int_{(a, b) \in \mathbb{R}^{2}}\left\|a \frac{1}{d} \nabla s_{x}(x)+b \frac{1}{d} \nabla s_{y}(x)\right\| \cdot\left\|a \frac{1}{d} \nabla s_{x}(y)+b \frac{1}{d} \nabla s_{y}(y)\right\| \frac{e^{-a^{2}-b^{2}}}{\pi} d a d b+O\left(\frac{1}{d}\right)= \\
\int_{(a, b) \in \mathbb{R}^{2}}\left\|a \frac{1}{d} \nabla s_{x}(x)\right\| \cdot\left\|b \frac{1}{d} \nabla s_{y}(y)\right\| \frac{e^{-a^{2}-b^{2}}}{\pi} d a d b+O\left(\frac{1}{d}\right)= \\
\frac{1}{d} \int_{a \in \mathbb{R}}\left\|a \nabla s_{x}(x)\right\| \frac{e^{-a^{2}}}{\sqrt{\pi}} d a \cdot \frac{1}{d} \int_{b \in \mathbb{R}}\left\|b \nabla s_{x}(x)\right\| \frac{e^{-b^{2}}}{\sqrt{\pi}} d b+O\left(\frac{1}{d}\right) .
\end{gathered}
$$

The last quantity equals $\frac{1}{d} \mathcal{N}_{d}^{1}(x) \frac{1}{d} \mathcal{N}_{d}^{1}(y)+O\left(\frac{1}{d}\right)$.

We then have

$$
\frac{1}{d} \mathcal{R}_{d}^{2}(x, y)=\frac{1}{d} \frac{\mathcal{N}_{d}^{2}(x, y)}{\mathcal{D}_{d}^{2}(x, y)}=\frac{1}{d} \frac{\mathcal{N}_{d}^{1}(x) \mathcal{N}_{d}^{1}(y)}{\mathcal{D}_{d}^{1}(x) \mathcal{D}_{d}^{1}(y)}+O\left(\frac{1}{d}\right)=\frac{1}{\sqrt{d}} \mathcal{R}_{d}^{1}(x) \frac{1}{\sqrt{d}} \mathcal{R}_{d}^{1}(y)+O\left(\frac{1}{d}\right)
$$

Let $\Delta_{d}$ be the neighborhood of the diagonal defined by

$$
\Delta_{d} \doteqdot\left\{\left(x_{1}, \ldots, x_{k}\right) \in \mathbb{R} \Sigma^{k} \mid \exists i \neq j, \mathbf{d}_{h}\left(x_{i}, x_{j}\right)<\frac{\log d}{C^{\prime} \sqrt{d}}\right\}
$$

where $C^{\prime}$ is the constant appearing in Theorem 2.2. The following result gives the estimate of the density function for points outside $\Delta_{d}$.

Lemma 4.3. Under the hypothesis of Theorem 1.3, for every $f \in C^{0}\left(\mathbb{R} \Sigma^{k}\right)$, the following asymptotics hold:

$$
\int_{\mathbb{R} \Sigma^{k} \backslash \Delta_{d}} f \frac{1}{\sqrt{d}^{k}} \mathcal{R}_{d}^{k}\left|d V_{h}\right|^{k}=\frac{1}{\sqrt{\pi}^{k}} \int_{\mathbb{R} \Sigma^{k} \backslash \Delta_{d}} f\left|d V_{h}\right|^{k}+\|f\|_{\infty} O\left(\frac{1}{d}\right)
$$

where the error $O\left(\frac{1}{d}\right)$ does not depend on $f$.

Proof. By Proposition 4.2, we have, for any $\left(x_{1}, \ldots, x_{k}\right) \in \mathbb{R} \Sigma^{k} \backslash \Delta_{d}$,

$$
\frac{1}{\sqrt{d}^{k}} \mathcal{R}_{d}^{k}\left(x_{1}, \ldots, x_{k}\right)=\prod_{i=1}^{k} \frac{1}{\sqrt{d}} \mathcal{R}_{d}^{1}\left(x_{i}\right)+O\left(\frac{1}{d}\right)
$$

By Theorem 5.38 in Section 5.6 (see also [11, Theorem 1.1]) we know that $\frac{1}{\sqrt{d}} \mathcal{R}_{d}^{1}\left(x_{i}\right)=\frac{1}{\sqrt{\pi}}+O\left(\frac{1}{d}\right)$, so we have the result.

We can prove the first claim of Theorem 1.4, that is:

Theorem 4.4. Let $(\mathcal{L}, h)$ be a positive real Hermitian line bundle over a real Riemann surface $\Sigma$. For every $f: \mathbb{R} \Sigma^{k} \rightarrow \mathbb{R}$ bounded function, we have

$$
\frac{1}{\sqrt{d}^{k}} \mathbb{E}\left[\nu_{s}^{k}\right](f)=\frac{1}{\sqrt{\pi}^{k}} \int_{\mathbb{R} \Sigma^{k}} f\left|d V_{h}\right|^{k}+\|f\|_{\infty} O\left(\frac{\log d}{\sqrt{d}}\right) .
$$

Proof. We denote by $\Delta_{d}$ the neighborhood of the diagonal defined by

$$
\Delta_{d} \doteqdot\left\{\left(x_{1}, \ldots, x_{k}\right) \in \mathbb{R} \Sigma^{k} \mid \exists i \neq j, \mathbf{d}_{h}\left(x_{i}, x_{j}\right)<\frac{\log d}{C^{\prime} \sqrt{d}}\right\}
$$

where $C^{\prime}$ is the constant appearing in Theorem 2.2. The volume of $\Delta_{d}$ is $O\left(\frac{\log d}{\sqrt{d}}\right)$.

Thanks to Proposition 3.5, we have

$$
\frac{1}{\sqrt{d}^{k}} \mathbb{E}\left[\tilde{\nu}_{s}^{k}\right](f)=\frac{1}{\sqrt{d}^{k}} \int_{\underline{x} \in \mathbb{R} \Sigma^{k}} f(\underline{x}) \mathcal{R}_{d}(\underline{x})\left|\mathrm{dV}_{h}\right|^{k}
$$


where $\tilde{\nu}_{s}^{k}$ is defined in Definition 3.1. By Lemma 4.3 we have

$$
\frac{1}{\sqrt{d}^{k}} \int_{\underline{x} \in \mathbb{R} \Sigma^{k} \backslash \Delta_{d}} f(\underline{x}) \mathcal{R}_{d}(\underline{x})\left|\mathrm{dV}_{h}\right|^{k}=\frac{1}{\sqrt{\pi}^{k}} \int_{\underline{x} \in \mathbb{R} \Sigma^{k} \backslash \Delta_{d}} f(\underline{x})\left|\mathrm{dV}_{h}\right|^{k}+O\left(\frac{1}{d}\right) .
$$

Moreover, by Theorem 4.1, the function $\frac{1}{\sqrt{d}^{k}} \mathcal{R}_{d}^{k}$ is uniformly bounded by a constant $C$, so that

$$
\frac{1}{\sqrt{d}^{k}} \int_{\underline{x} \in \Delta_{d}} f(\underline{x}) \mathcal{R}_{d}^{k}(\underline{x})\left|\mathrm{dV}_{h}\right|^{k} \leq C\|f\|_{\infty} \operatorname{Vol}\left(\Delta_{d}\right)=\|f\|_{\infty} O\left(\frac{\log d}{\sqrt{d}}\right) .
$$

Putting together the integral over $\mathbb{R} \Sigma^{k} \backslash \Delta_{d}$ and over $\Delta_{d}$, we obtain

$$
\frac{1}{\sqrt{d}^{k}} \mathbb{E}\left[\tilde{\nu}_{s}^{k}\right](f)=\frac{1}{\sqrt{\pi}^{k}} \int_{\underline{x} \in \mathbb{R} \Sigma^{k}} f(\underline{x})\left|\mathrm{dV}_{h}\right|^{k}+\|f\| O\left(\frac{\log d}{\sqrt{d}}\right) .
$$

To conclude we must compare the moment $\mathbb{E}\left[\nu_{s}^{k}\right]$ with the modified moment $\mathbb{E}\left[\tilde{\nu}_{s}^{k}\right]$. By Proposition 3.2 , the quantity $\mathbb{E}\left[\nu_{s}^{k}\right]-\mathbb{E}\left[\tilde{\nu}_{s}^{k}\right]$ is a sum of terms of the form $\mathbb{E}\left[\tilde{\nu}_{s}^{m}\right]$ with $m<k$. This implies that $\left(\mathbb{E}\left[\nu_{s}^{k}\right]-\mathbb{E}\left[\tilde{\nu}_{s}^{k}\right]\right)(f)=O\left(\sqrt{d}^{k-1}\right)$ and then

$$
\frac{1}{\sqrt{d}^{k}} \mathbb{E}\left[\nu_{s}^{k}\right](f)=\frac{1}{\sqrt{\pi}^{k}} \int_{\underline{x} \in \mathbb{R} \Sigma^{k}} f(\underline{x})\left|\mathrm{dV}_{h}\right|^{k}+\|f\| O\left(\frac{\log d}{\sqrt{d}}\right) .
$$

\subsection{Proof of Theorems 1.1, 1.4 and 1.5}

This section is devoted to the proof of the main theorems. We follow the notation of Section 3. Remember that $\mathcal{R}_{d}^{k}$ is the density function given by Proposition 3.5. We need the following

Definition 4.5. We denote by $\mathbf{d}_{h}$ the distance on $\mathbb{R} \Sigma^{k}$ induced by $h$.

- Let $U_{d}$ be the union $\cup_{a<b} U_{d}^{a, b}$, where $U_{d}^{a, b}$ is the set

$$
U_{d}^{a, b} \doteqdot\left\{\left(x_{1}, \ldots, x_{k}\right) \in \mathbb{R} \Sigma^{k} \mid \mathbf{d}_{h}\left(x_{i}, x_{j}\right) \leq \frac{\log d}{C^{\prime} \sqrt{d}} \Rightarrow(i, j)=(a, b)\right\}
$$

with $C^{\prime}$ being the constant appearing in Theorem 2.2.

Equivalently, a point $\left(x_{1}, \ldots, x_{k}\right)$ lies in $U_{d}^{a, b}$ if and only if the distance between every pair of points $\left(x_{i}, x_{j}\right), i \neq j \in\{1, \ldots, k\}$, is bigger than $\frac{\log d}{C^{\prime} \sqrt{d}}$, except at most the pair $\left(x_{a}, x_{b}\right)$.

- For any $1 \leq a<b \leq k$, we denote by $j_{a b}: \mathbb{R} \Sigma^{k-1} \hookrightarrow \mathbb{R} \Sigma^{k}$ the inclusion

$$
\left(x_{1}, \ldots, x_{a}, \ldots, \hat{x}_{b}, \ldots, x_{k}\right) \mapsto\left(x_{1}, \ldots, x_{a}, \ldots, x_{a}, \ldots, x_{k}\right),
$$

so that the image $j_{a b}\left(\mathbb{R} \Sigma^{k-1}\right)$ is equal to

$$
\left\{\left(x_{1}, \ldots, x_{k}\right) \in \mathbb{R} \Sigma^{k} \mid x_{a}=x_{b}\right\} .
$$

Lemma 4.6. Let $U_{d}$ be the set defined in Definition 4.5. Then

$$
\operatorname{Vol}_{h}\left(\mathbb{R} \Sigma^{k} \backslash U_{d}\right)=O\left(\frac{(\log d)^{2}}{d}\right) .
$$

Proof. By definition, $U_{d}$ is the set of points $\left(x_{1}, \ldots, x_{k}\right) \in \mathbb{R} \Sigma^{k}$ such that the distance between each pair of points $x_{i}, x_{j}, 1 \leq i<j \leq k$, is bigger than $\frac{\log d}{C^{\prime} \sqrt{d}}$, except at most one pair.

Its complement $\mathbb{R} \Sigma^{k} \backslash U_{d}$ is then formed by points $\left(x_{1}, \ldots, x_{k}\right) \in \mathbb{R} \Sigma^{k}$ such that there exist four indices $i, t, s, j \in\{1, \ldots, k\}, i \neq t, i \neq j, s \neq j$, with the property that $\mathbf{d}_{h}\left(x_{i}, x_{t}\right) \leq \frac{\log d}{C^{\prime} \sqrt{d}}$ and $\mathbf{d}_{h}\left(x_{s}, x_{j}\right) \leq \frac{\log d}{C^{\prime} \sqrt{d}}$. (Note that the indices $t$ and $s$ could be equal).

Fix such four indices $i, t, s, j$, and denote by $V_{d}^{i, t, s, j}$ the set of points $\left(x_{1}, \ldots, x_{k}\right) \in \mathbb{R} \Sigma^{k}$ such that $\mathbf{d}_{h}\left(x_{i}, x_{t}\right) \leq \frac{\log d}{C^{\prime} \sqrt{d}}$ and $\mathbf{d}_{h}\left(x_{s}, x_{j}\right) \leq \frac{\log d}{C^{\prime} \sqrt{d}}$. The volume of $V_{d}^{i, t, s, j}$ is then $O\left(\frac{(\log d)^{2}}{d}\right)$. We then obtain

$$
\operatorname{Vol}\left(\mathbb{R} \Sigma^{k} \backslash U_{d}\right) \leq \sum_{i, t, s, j} \operatorname{Vol}\left(V_{d}^{i, t, s, j}\right)=O\left(\frac{(\log d)^{2}}{d}\right) .
$$


We formulate an uniform estimate of the density function $\mathcal{R}_{d}^{k}$ over $U_{d}$. This estimate, together with Theorem 4.1, will imply Theorem 1.4.

Proposition 4.7. Under the hypothesis of Theorem 1.3 and using the notations of Definition 4.5, there exists an universal constant $M^{\prime}$ such that, for every $f \in C^{0}\left(\mathbb{R} \Sigma^{k}\right)$, the following asymptotics hold:

$$
\frac{1}{\sqrt{d}^{k}} \int_{U_{d}} f \mathcal{R}_{d}^{k}\left|d V_{h}\right|^{k}=\frac{1}{\sqrt{\pi}^{k}} \int_{U_{d}} f\left|d V_{h}\right|^{k}+\frac{1}{\sqrt{\pi}^{k-2}} \sum_{a<b} \frac{M^{\prime}}{\sqrt{d}} \int_{\mathbb{R} \Sigma^{k-1}} j_{a b}^{*} f\left|d V_{h}\right|^{k-1}+o\left(\frac{1}{\sqrt{d}}\right)
$$

The error term o $\left(\frac{1}{\sqrt{d}}\right)$ is bounded from above by

$$
\|f\|_{\infty}\left(O\left(\frac{1}{\sqrt{d}^{1+\alpha}}\right)+\omega_{f}\left(\frac{1}{\sqrt{d}^{\alpha}}\right) O\left(\frac{1}{\sqrt{d}}\right)\right)
$$

for any $\alpha \in(0,1)$, where $\omega_{f}(\cdot)$ is the modulus of continuity of $f$. The errors $O\left(\frac{1}{\sqrt{d}^{1+\alpha}}\right)$ and $O\left(\frac{1}{\sqrt{d}}\right)$ do not depend on $f$. Moreover $M \doteqdot M^{\prime}+\frac{1}{\sqrt{\pi}}$ is positive.

We will prove this Proposition in Section 5.6. We now deduce Theorem 1.4.

Proof of Theorem 1.4. We have to prove the second claim of the theorem, that is the better asymptotic estimate in the case of $f$ continuous function. The first claim was proved in Theorem 4.4. We start by computing the modified moment $\mathbb{E}\left[\tilde{\nu}_{s}^{k}\right]$, see Definition 3.1. Thanks to Proposition 3.5, we have

$$
\frac{1}{\sqrt{d}^{k}} \mathbb{E}\left[\tilde{\nu}_{s}^{k}\right](f)=\frac{1}{\sqrt{d}^{k}} \int_{\mathbb{R} \Sigma^{k}} f(\underline{x}) \mathcal{R}_{d}^{k}(\underline{x})\left|\mathrm{dV}_{h}\right|^{k}
$$

We divide the integration domain into two parts, namely the set $U_{d}$ defined in Definition 4.5 and its complement $\mathbb{R} \Sigma^{k} \backslash U_{d}$.

- Proposition 4.7 implies that $\frac{1}{\sqrt{d}^{k}} \int_{U_{d}} f(\underline{x}) \mathcal{R}_{d}^{k}(\underline{x})\left|\mathrm{dV}_{h}\right|^{k}$ is equal to

$$
\frac{1}{\sqrt{\pi}^{k}} \int_{U_{d}} f(\underline{x})\left|\mathrm{dV}_{h}\right|^{k}+\frac{1}{\sqrt{\pi}^{k-2}} \sum_{a<b} \frac{M^{\prime}}{\sqrt{d}} \int_{\mathbb{R} \Sigma^{k-1}} j_{a b}^{*} f\left|\mathrm{dV}_{h}\right|^{k-1}+o\left(\frac{1}{\sqrt{d}}\right)
$$

where the error term $o\left(\frac{1}{\sqrt{d}}\right)$ and the universal constant $M^{\prime}$ are as in Proposition 4.7.

- Theorem 4.1 and Lemma 4.6 imply

$$
\frac{1}{\sqrt{d}^{k}} \int_{\mathbb{R} \Sigma^{k} \backslash U_{d}} f(\underline{x}) \mathcal{R}_{d}^{k}(\underline{x})\left|\mathrm{dV}_{h}\right|^{k} \leq \operatorname{Vol}\left(\mathbb{R} \Sigma^{k} \backslash U_{d}\right)\|f\|_{\infty} O(1)=\|f\|_{\infty} O\left(\frac{(\log d)^{2}}{d}\right)
$$

where the error $O\left(\frac{(\log d)^{2}}{d}\right)$ does not depend on $f$.

Remark that the integral over $\mathbb{R} \Sigma^{k} \backslash U_{d}$ is then negligible compared to the integral over its complement $U_{d}$. This implies that the integral over the cartesian product $\mathbb{R} \Sigma^{k}$ is equal to the integral over $U_{d}$, up to an error term. In other words, putting together the integral over $\mathbb{R} \Sigma^{k} \backslash U_{d}$ and over $U_{d}$, we obtain

$$
\frac{1}{\sqrt{d}^{k}} \mathbb{E}\left[\tilde{\nu}_{s}^{k}\right](f)=\frac{1}{\sqrt{\pi}^{k}} \int_{\mathbb{R} \Sigma^{k}} f(\underline{x})\left|\mathrm{d} \mathrm{V}_{h}\right|^{k}+\frac{1}{\sqrt{\pi}^{k-2}} \sum_{a<b} \frac{M^{\prime}}{\sqrt{d}} \int_{\mathbb{R} \Sigma^{k-1}} j_{a b}^{*} f\left|\mathrm{dV}_{h}\right|^{k-1}+o\left(\frac{1}{\sqrt{d}}\right)
$$

where the error $o\left(\frac{1}{\sqrt{d}}\right)$ is as in Proposition 4.7 .

To conclude we must compare the moment $\mathbb{E}\left[\nu_{s}^{k}\right]$ with the modified moment $\mathbb{E}\left[\tilde{\nu}_{s}^{k}\right]$. By Proposition 3.2 we have

$$
\frac{1}{\sqrt{d}^{k}}\left(\nu_{s}^{k}(f)-\tilde{\nu}_{s}^{k}(f)\right)=\frac{1}{\sqrt{d}^{k}} \sum_{I \in \widetilde{\mathcal{P}}_{k}} \tilde{\nu}_{s}^{m_{I}}\left(j_{I}^{*} f\right)
$$

see Section 3.1 for definition of $\widetilde{\mathcal{P}}_{k}$ and of $j_{I}$. By Eq. (1) applied to $m_{I} \leq k-2$, we get

$$
\frac{1}{\sqrt{d}^{k}} \mathbb{E}\left[\tilde{\nu}_{s}^{m_{I}}\right]\left(j_{I}^{*} f\right)=\|f\|_{\infty} O\left(\frac{1}{\sqrt{d}^{k-m_{I}}}\right) .
$$


We then obtain

$$
\frac{1}{\sqrt{d}^{k}}\left(\mathbb{E}\left[\nu_{s}^{k}\right]-\mathbb{E}\left[\tilde{\nu}_{s}^{k}\right]\right)(f)=\frac{1}{\sqrt{d}^{k}} \sum_{1 \leq a<b \leq k} \mathbb{E}\left[\tilde{\nu}_{s}^{k-1}\right]\left(j_{a b}^{*} f\right)+\|f\|_{\infty} O\left(\frac{1}{d}\right) .
$$

We can use Eq. (1), for the modified moments $\mathbb{E}\left[\tilde{\nu}_{s}^{k-1}\right]\left(j_{a b}^{*} f\right)$ and we obtain:

$$
\frac{1}{\sqrt{d}^{k}}\left(\mathbb{E}\left[\nu_{s}^{k}\right]-\mathbb{E}\left[\tilde{\nu}_{s}^{k}\right]\right)(f)=\frac{1}{\sqrt{\pi}^{k-1} \sqrt{d}} \sum_{1 \leq a<b \leq k} \int_{\mathbb{R} \Sigma^{k-1}} j_{a b}^{*} f\left|\mathrm{dV}_{h}\right|^{k-1}+\|f\|_{\infty} O\left(\frac{1}{d}\right) .
$$

Putting $\frac{1}{\sqrt{d}^{k}} \mathbb{E}\left[\tilde{\nu}_{s}^{k}\right](f)$ on the right hand side and using again Eq. (1) we obtain

$$
\frac{1}{\sqrt{d}^{k}} \mathbb{E}\left[\nu_{s}^{k}\right](f)=\frac{1}{\sqrt{\pi}^{k}} \int_{\mathbb{R} \Sigma} f\left|\mathrm{dV}_{h}\right|^{k}+\frac{M}{\sqrt{\pi}^{k-2} \sqrt{d}} \sum_{a<b} \int_{j_{a b}\left(\mathbb{R} \Sigma^{k-1}\right)} f_{\mid\left\{x_{a}=x_{b}\right\}}\left|\mathrm{dV} \mathrm{V}_{h}\right|^{k-1}+o\left(\frac{1}{\sqrt{d}}\right)
$$

where $M=M^{\prime}+\frac{1}{\sqrt{\pi}}$ and where the error term is as in Proposition 4.7. The constant $M$ is positive thanks to Proposition 4.7.

Proof of Theorem 1.5. We prove the theorem in the case of $f: \mathbb{R} \Sigma \rightarrow \mathbb{R}$ continuous. The case of $f$ bounded follows the same lines. Let $f$ be a continuous function on $\mathbb{R} \Sigma$. It induces a continuous function on $\mathbb{R} \Sigma^{l}$ for every $l \in \mathbb{N}$ defined by

$$
\left(x_{1}, \ldots, x_{l}\right) \in \mathbb{R} \Sigma^{l} \mapsto f\left(x_{1}\right) \cdots f\left(x_{l}\right) \in \mathbb{R} .
$$

With a slight abuse of notation we still denote this function by $f$. For any $l \in \mathbb{N}$, we define

$$
\mathcal{E}_{l}(f) \doteqdot \frac{1}{\sqrt{d}^{l-1}}\left(\mathbb{E}\left[\nu_{s}^{l}\right](f)-\mathbb{E}\left[\nu_{s}\right]^{l}(f)\right)
$$

By Theorem 5.38, we have

$$
\frac{1}{\sqrt{d}} \mathbb{E}\left[\nu_{s}\right](f)=\frac{1}{\sqrt{\pi}} \int_{\mathbb{R} \Sigma} f\left|\mathrm{dV}_{h}\right|+O\left(\frac{1}{d}\right)
$$

so that

$$
\frac{1}{\sqrt{d}^{l}} \mathbb{E}\left[\nu_{s}\right]^{l}(f)=\frac{1}{\sqrt{\pi}^{l}}\left(\int_{\mathbb{R} \Sigma} f\left|\mathrm{dV}_{h}\right|\right)^{l}+O\left(\frac{1}{d}\right)
$$

for any $l \in \mathbb{N}$. Combining this with Theorem 1.4, we have that for any $l$,

$$
\mathcal{E}_{l}(f)=\frac{M}{\sqrt{\pi}^{l-2}} \sum_{1 \leq a<b \leq l} \int_{\mathbb{R} \Sigma^{l-1}} j_{a b}^{*} f\left|\mathrm{dV}_{h}\right|^{l-1}+o(1)=\frac{M l(l-1)}{2 \sqrt{\pi}^{l-2}}\left(\int_{\mathbb{R} \Sigma} f\left|\mathrm{dV}_{h}\right|\right)^{l-1}+o(1)
$$

where the error term $o(1)$ is bounded from above by

$$
\|f\|_{\infty}\left(O\left(\frac{1}{\sqrt{d}^{\alpha}}\right)+\omega_{f}\left(\frac{1}{\sqrt{d}^{\alpha}}\right) O(1)\right)
$$

for any $\alpha \in(0,1)$, where $\omega_{f}(\cdot)$ is the modulus of continuity of $f$. Moreover the errors $O\left(\frac{1}{\sqrt{d}^{\alpha}}\right)$ and $O(1)$ do not depend on $f$.

We then have

$$
\begin{gathered}
\frac{1}{\sqrt{d}^{k-1}} \mathbb{E}\left[\left(\nu_{s}-\mathbb{E}\left[\nu_{s}\right]\right)^{k}\right](f)=\mathbb{E}\left[\sum_{l=0}^{k}(-1)^{k-l}\left(\begin{array}{c}
k \\
l
\end{array}\right) \frac{1}{\sqrt{d}^{l-1}} \nu_{k}^{l}(f) \frac{1}{\sqrt{d}^{k-l}} \mathbb{E}\left[\nu_{s}(f)\right]^{k-l}\right] \\
=\sum_{l=0}^{k}(-1)^{k-l}\left(\begin{array}{c}
k \\
l
\end{array}\right) \frac{1}{\sqrt{d}^{l-1}} \mathbb{E}\left[\nu_{s}^{l}(f)\right] \frac{1}{\sqrt{d}^{k-l}} \mathbb{E}\left[\nu_{s}(f)\right]^{k-l} \\
=\sum_{l=0}^{k}(-1)^{k-l}\left(\begin{array}{c}
k \\
l
\end{array}\right)\left(\frac{1}{\sqrt{d}^{l-1}} \mathbb{E}\left[\nu_{s}(f)\right]^{l}+\mathcal{E}_{l}(f)\right) \frac{1}{\sqrt{d}^{k-l}} \mathbb{E}\left[\nu_{s}(f)\right]^{k-l}
\end{gathered}
$$




$$
=\sum_{l=0}^{k}(-1)^{k-l}\left(\begin{array}{l}
k \\
l
\end{array}\right)\left(\frac{1}{\sqrt{d}^{k-1}} \mathbb{E}\left[\nu_{s}(f)\right]^{k}+\mathcal{E}_{l}(f) \frac{1}{\sqrt{d}^{k-l}} \mathbb{E}\left[\nu_{s}(f)\right]^{k-l}\right)
$$

By the formula $\sum_{l=0}^{k}(-1)^{l}\left(\begin{array}{l}k \\ l\end{array}\right)=0$ we have that Eq. (4) equals

$$
\sum_{l=0}^{k}(-1)^{k-l}\left(\begin{array}{l}
k \\
l
\end{array}\right) \mathcal{E}_{l}(f) \frac{1}{\sqrt{d}^{k-l}} \mathbb{E}\left[\nu_{s}(f)\right]^{k-l} .
$$

Now we use Eq. (3) for the term $\mathcal{E}_{l}(f)$ and Eq. (2) for the term $\frac{1}{\sqrt{d}^{k-l}} \mathbb{E}\left[\nu_{s}(f)\right]^{k-l}$ and we obtain that Eq. (5) is equal to

$$
\sum_{l=0}^{k}(-1)^{k-l}\left(\begin{array}{c}
k \\
l
\end{array}\right) l(l-1) \frac{M}{2 \sqrt{\pi}^{k-2}}\left(\int_{\mathbb{R} \Sigma} f\left|\mathrm{dV}_{h}\right|\right)^{k-1}+o(1) .
$$

Now, $\sum_{l=0}^{k}(-1)^{k-l}\left(\begin{array}{l}k \\ l\end{array}\right) l(l-1)$ equals $\left.\frac{d^{2}}{d x^{2}}\right|_{x=1}(x-1)^{k}$ and this vanishes as $k>2$.

Proof of Theorem 1.1. Let $A$ be a measurable subset of $\mathbb{R} \Sigma$ of positive volume. We have

$$
\left|\#\left(Z_{s} \cap A\right)-\mathbb{E}\left[\#\left(Z_{s} \cap A\right)\right]\right|>c(d) \sqrt{d} \Leftrightarrow\left|\#\left(Z_{s} \cap A\right)-\mathbb{E}\left[\#\left(Z_{s} \cap A\right)\right]\right|^{k}>c(d)^{k} \sqrt{d}^{k}
$$

so that by Markov's inequality we obtain

$$
\mu\left\{\left|\#\left(Z_{s} \cap A\right)-\mathbb{E}\left[\#\left(Z_{s} \cap A\right)\right]\right|>c(d) \sqrt{d}\right\} \leqslant \frac{\mathbb{E}\left[\left|\#\left(Z_{s} \cap A\right)-\mathbb{E}\left[\#\left(Z_{s} \cap A\right)\right]\right|^{k}\right]}{c(d)^{k} \sqrt{d}^{k}}
$$

By Theorem 1.5 respectively for $f=\mathbb{1}_{A}$ and $f=1$, we have the result.

\section{Olver multispaces and proof of Theorem 4.1}

This section is devoted to the proof of the boundedness result used in the proof of Theorem 1.4, namely Theorem 4.1. Olver multispaces and divided differences coordinates play an important role. We recall these tools in Section 5.1.

The main difficulty in Theorem 4.1 is to understand the behaviour of the density function $\mathcal{R}_{d}^{k}$ near the diagonal, see Remark 3.13. For this purpose, we will study all the possible ways a point $\left(x_{1}, \ldots, x_{k}\right) \in$ $\mathbb{R} \Sigma^{k} \backslash \Delta$ can converge to the diagonal. This leads us to consider labelled graph with $k$ vertices. Each vertex represents a point $x_{i} \in \mathbb{R} \Sigma$ and we put an edge between two vertex if and only if we allow the points associated with these vertices to collapse each other, see Definition 5.10.

\subsection{Olver multispaces}

We briefly recall the results of [19]. Let $M$ an $n$-dimensional smooth (not necessarly connected or closed) manifold and $C$ and $C^{\prime}$ two curves in $M$ passing at $z \in M$. We introduce local coordinates $\left(x, u_{1}, \ldots, u_{n-1}\right)=(x, u)$ around $z$ such that, locally, the curve $C$ is a graph $\{u=f(x)\}$ and the curve $C^{\prime}$ a graph $\{u=g(x)\}$, for smooth maps $f, g$.

Definition 5.1. Let $z=\left(x_{0}, u_{0}\right)$ be a point in a smooth manifold $M$ and $C=\{u=f(x)\}, C^{\prime}=$ $\{u=g(x)\}$ be two curves passing at $z$. We say that $C$ and $C^{\prime}$ have the same $k$-jet at $z$ if and only if $f^{(i)}\left(x_{0}\right)=g^{(i)}\left(x_{0}\right)$ for every $i \in\{0, \ldots, k\}$. We then write $\left.j_{k} C\right|_{z}=\left.j_{k} C\right|_{z} ^{\prime}$.

\subsubsection{Pointed manifolds}

A $(k+1)$-pointed manifold is an object $\mathbf{M}=\left(M ; z_{0}, \ldots, z_{k}\right)$ consisting of a smooth manifold $M$ and $(k+1)$ not necessarily distinct points $z_{0}, \ldots, z_{k} \in M$ thereon. Given $\mathbf{M}$, we let $\# i=\#\left\{j \mid z_{j}=z_{i}\right\}$ denote the number of points which coincide with the $i$-th one. 


\subsubsection{Multi-contact}

Given a manifold $M$, we let $\mathcal{C}^{(k)}=\mathcal{C}^{(k)}(M)$ denote the set of all $(k+1)$-pointed curves contained in $X$. In [19], Olver defined an equivalence relation on the space of multi-pointed curves that generalizes the jet equivalence relation at a single point.

Definition 5.2. Two $(k+1)$-pointed curves

$$
\mathbf{C}=\left(C ; z_{0}, \ldots, z_{k}\right) \quad \widetilde{\mathbf{C}}=\left(\tilde{C} ; \tilde{z}_{0}, \ldots, \tilde{z}_{k}\right)
$$

have $k$-order multi-contact if and only if

$$
z_{i}=\tilde{z}_{i} \quad \text { and }\left.\quad j_{\# i-1} C\right|_{z_{i}}=\left.j_{\# i-1} \widetilde{C}\right|_{z_{i}}
$$

for each $i=0, \ldots, k$. The $k$-order multi-space, denoted by $M^{(k)}$, is the set of equivalence classes of $(k+1)$-pointed curves in $M$ under the equivalence relation of $k$-th order multi-contact. The equivalence class of an $(k+1)$-pointed curve $\mathbf{C}$ is called its $k$-th order multi-jet, and denoted $j_{k} \mathbf{C} \in M^{(k)}$.

In [19] Olver proved the following theorem:

Theorem 5.3. If $M$ is a smooth manifold of dimension $n$, then its $k$-th order multispace $M^{(k)}$ is a smooth manifold of dimension $(k+1) n$, which contains the off diagonal part $M^{\diamond(k+1)} \doteqdot\left\{\left(z_{0}, \ldots, z_{k}\right) \in\right.$ $\left.M^{k+1} \mid z_{i} \neq z_{j} \forall i \neq j\right\}$ of the Cartesian product space as an open, dense subset, and the $k$-order jet space $J^{k}$ as a smooth submanifold.

Remark 5.4. For a one-dimensional variety, we have $M^{(k)} \simeq M^{k+1}$ where the canonical isomorphism is given by the forgetful map $\left[C ; z_{0}, \ldots, z_{k}\right] \mapsto\left(z_{0}, \ldots, z_{k}\right)$ with the inverse given by $\left(z_{0}, \ldots, z_{k}\right) \mapsto\left[M ; z_{0}, \ldots, z_{k}\right]$.

\subsubsection{Divided differences}

Olver proved Theorem 5.3 by providing an atlas. This atlas is formed by local charts given by divided differences.

Definition 5.5. Let $\left(C ; z_{0}, \ldots, z_{k}\right)$ be a $(k+1)$-pointed curve in a smooth manifold of dimension $n$. We introduce local coordinates $\left(x, u_{1}, \ldots, u_{n-1}\right)=(x, u)$ and we suppose that the curve $C$ is the graph $\{u=f(x)\}$ of a smooth function $f$, so that $z_{i}=\left(x_{i}, f\left(x_{i}\right)\right)$. We define the divided differences of $\left(C ; z_{0}, \ldots, z_{k}\right)$ recursively by setting $\left[z_{0}\right]_{C}=f\left(x_{0}\right)$ and

$$
\left[z_{0} \ldots z_{i-1} z_{i}\right]_{C}=\lim _{x \rightarrow x_{i}} \frac{\left[z_{0} \ldots z_{i-2} z\right]_{C}-\left[z_{0} \ldots z_{i-1}\right]_{C}}{x-x_{i-1}}
$$

for any $i \in\{1, \ldots k\}$. For example, $\left[z_{0} z_{1}\right]_{C}=\lim _{x \rightarrow x_{1}} \frac{z-z_{0}}{x-x_{0}}$. When taking the limit, the points $z=(x, f(x))$ must lie on the curve $C$.

Olver proved that two $(k+1)$-pointed curves $\left(C ; z_{0}, \ldots, z_{k}\right)$ and $\left(C^{\prime} ; z_{0}, \ldots, z_{k}\right)$ represent the same element in $M^{(k)}$ if and only if they have same divided differences, see [19, Theorem 3.4].

The following lemma will be useful for the local computations in Sections 5.4 and 5.5.

Lemma 5.6. Let $\underline{T}=\left(T_{1}, \ldots, T_{k}\right) \in \mathbb{R}^{k}$ and $f \in C^{k}(\mathbb{R})$ be a $C^{k}$-function and $C=\operatorname{graph}(f)$. Then

$$
\left[f\left(T_{1}\right) \ldots f\left(T_{k}\right)\right]_{C}=\sum_{i=1}^{k} \sum_{r=0}^{\# T_{i}-1} c_{\underline{T}, i, r} f^{(r)}\left(T_{i}\right)
$$

where $c_{\underline{T}, i, r}$ is a rational function in the distances $T_{s}-T_{t}, 1 \leq s<t \leq k$ of non positive degree $r-k+1$. Here, $f^{(r)}(T)$ is the $r$-th derivative of $f$ at $T$.

In the sequel we will omit $C$ in the notation of divided differences, that is we write $[\cdots]$ instead of $[\cdots]_{C}$.

Proof. By induction on $k$. If $k=1$ we have $\left[f\left(T_{1}\right)\right]=f\left(T_{1}\right)$.

By definition, we have

$$
\left[f\left(T_{1}\right) \ldots f\left(T_{k-1}\right) f\left(T_{k}\right)\right]=\lim _{T \rightarrow T_{k}} \frac{\left[f\left(T_{1}\right) \ldots f\left(T_{k-2}\right) f(T)\right]-\left[f\left(T_{1}\right) \ldots f\left(T_{k-1}\right)\right]}{T-T_{k-1}}
$$


and by induction hypothesis we have

$$
\left[f\left(T_{1}\right) \ldots f\left(T_{k-1}\right)\right]=\sum_{i=1}^{k-1} \sum_{r=0}^{\# T_{i}-1} c_{T^{\prime}, i, r} f^{(r)}\left(T_{i}\right)
$$

with $\underline{T^{\prime}}=\left(T_{1}, \ldots, T_{k-1}\right) \in \mathbb{R}^{k-1}$. If $T_{k} \neq T_{k-1}$, then

$$
\left[f\left(T_{1}\right) \ldots f\left(T_{k-1}\right) f\left(T_{k}\right)\right]=\frac{\left[f\left(T_{1}\right) \ldots f\left(T_{k-2}\right) f\left(T_{k}\right)\right]}{T_{k}-T_{k-1}}-\frac{\left[f\left(T_{1}\right) \ldots f\left(T_{k-1}\right)\right]}{T_{k}-T_{k-1}}
$$

and by induction we have the result.

If $T_{k}=T_{k-1}$, then

$$
\left[f\left(T_{1}\right) \ldots f\left(T_{k-1}\right) f\left(T_{k}\right)\right]=\frac{d}{d T_{k-1}}\left[f\left(T_{1}\right) \ldots f\left(T_{k-1}\right)\right]=\frac{d}{d T_{k-1}} \sum_{i=1}^{k-1} \sum_{r=0}^{\# T_{i}-1} c_{\underline{T}^{\prime}, i, r} f^{(r)}\left(T_{i}\right)
$$

and, again, by induction hypothesis we have the result.

\subsection{Extension of the density function $\mathcal{R}_{d}^{k}$}

In this subsection we introduce an incidence manifold that will play a key role in the proof of Theorem 4.1 and of the following:

Theorem 5.7. For large d, the density function $\mathcal{R}_{d}^{k}: \mathbb{R} \Sigma^{k} \backslash \Delta \rightarrow \mathbb{R}$ in Proposition 3.5 can be extended to a smooth function $\mathbb{R} \Sigma^{k} \rightarrow \mathbb{R}$ that we still denote by $\mathcal{R}_{d}^{k}$. Moreover, $\mathcal{R}_{d}^{k}$ vanishes on the diagonal $\Delta$.

\subsubsection{Extension of the incidence manifold}

Let $\mathbb{R} \mathcal{L}^{d}$ be the real locus of the total space of the line bundle $\mathcal{L}^{d} \rightarrow \Sigma$. It is an open manifold of real dimension 2. For $k \in \mathbb{N}$, we take its multi-space $\left(\mathbb{R} \mathcal{L}^{d}\right)^{(k-1)}$, see Section 5.1.

Consider the map

$$
e v: \mathbb{R} H^{0}\left(\Sigma ; \mathcal{L}^{d}\right) \times \mathbb{R} \Sigma^{k} \rightarrow\left(\mathbb{R} \mathcal{L}^{d}\right)^{(k-1)}
$$

defined by

$$
\left(s, x_{1}, \ldots, x_{k}\right) \mapsto\left(\operatorname{graph}(s) ; s\left(x_{1}\right), \ldots, s\left(x_{k}\right)\right) .
$$

Let $\mathbf{Z}$ be the set of points in $\left(\mathbb{R} \mathcal{L}^{d}\right)^{(k-1)}$ which represent the $k$-pointed curves $\left(\mathbb{R} \Sigma ; x_{1}, \ldots, x_{k}\right)$, where $\mathbb{R} \Sigma$ is viewed as the zero section of $\mathbb{R} \mathcal{L}^{d}$ and $x_{1}, \ldots, x_{k} \in \mathbb{R} \Sigma$.

Definition 5.8. We denote the set $e v^{-1}(\mathbf{Z})$ by $\mathcal{I}$ and call it the incidence manifold.

Remark 5.9. For any $\left(x_{1}, \ldots, x_{k}\right) \in \mathbb{R} \Sigma^{k} \backslash \Delta$ we have $\left(s, x_{1}, \ldots, x_{k}\right) \in e v^{-1}(\mathbf{Z})$ if and only if $s\left(x_{i}\right)=0$ for any $i \in\{1, \ldots, k\}$. Hence, the Definition 5.8 extends the definition of the incidence manifold given in Section 3.2.

\subsection{Local equations of the incidence manifold}

We give local equations for the incidence manifold $\mathcal{I}$ defined in Definition 5.8. These local equations allow us to find a new local expression for the density function $\mathcal{R}_{d}^{k}$. We will use the divided differences notation (see Section 5.1, Definition 5.5).

\subsubsection{A partition of $\mathbb{R} \Sigma^{k}$}

Let $\Theta_{k}$ be the set of all labelled graphs with exactly $k$ vertices, labelled by $\{1, \ldots, k\}$. Let $d$ be any positive integer, we will associate a graph in $\Theta_{k}$ to any point $\left(x_{1}, \ldots, x_{k}\right) \in \mathbb{R} \Sigma^{k}$. This graph is contructed as follows: we put an edge between the $i$-th vertex and $j$-th vertex if and only if the distance $\mathbf{d}_{h}\left(x_{i}, x_{j}\right)$ between $x_{i}$ and $x_{j}$ is smaller or equal than $\frac{1}{\sqrt{d}}$.

Definition 5.10. - Given an integer $d \in \mathbb{N}$ and a point $\underline{x} \in \mathbb{R} \Sigma^{k}$ we say that $\underline{x} \in \Gamma_{d}$ if and only if its associated graph is $\Gamma$. We will refer to $\Gamma_{d}$ as a graph subset. 
- We call origin of a connected component of such a graph the point of the connected component with the smallest label.

- If $\Gamma$ has $m$ connected components $\Gamma^{1}, \ldots, \Gamma^{m}$ and each connected component $\Gamma^{i}$ has $k_{i}$ vertices, then we write $\left\{x_{1}^{i}, \ldots, x_{k_{i}}^{i}\right\}$ for the corresponding points of the connected component. We then have $\left\{x_{p}^{i}\right\}_{p=1, \ldots, k_{i}}^{i=1, \ldots, m}=\left\{x_{1}, \ldots, x_{k}\right\}$.

We say that $\left\{x_{1}^{i}, \ldots, x_{k_{i}}^{i}\right\}$ is a connected component of $\underline{x}$ and that $x_{p}^{i}$ is a vertex of $\underline{x}$.

Remark 5.11. - For any $d \in \mathbb{N}$, the graph subsets $\left\{\Gamma_{d}\right\}_{\Gamma \in \Theta_{k}}$ give a cover of $\mathbb{R} \Sigma^{k}$.

- The fact that two point $x_{i}$ and $x_{j}$ lie in the same connected components implies that the distance between these two points is smaller or equal than $\frac{k}{\sqrt{d}}$.

\subsubsection{Generalized evaluation maps}

Fix a $k$-labelled graph $\Gamma \in \Theta_{k}$ with $m$ connected components. We write $\left\{x_{1}^{i}, \ldots, x_{k_{i}}^{i}\right\}_{1 \leq i \leq m}$ for the connected components of a point $\underline{x}$ that lies in $\Gamma_{d}$. We take real normal coordinates $U_{i}$ around $x_{1}^{i}$, see Section 2.2.2. We identify each point $x_{p}^{i}$ with the respective coordinate in the chart $U_{i}$. We recall that with a real normal chart comes together with a real trivialization of $\mathbb{R} \mathcal{L}_{\mid U}^{d}$, see Section 2.2.2.

Definition 5.12. Given a point $\underline{x} \in \Gamma_{d}$ in normal coordinates around the origin $x_{1}^{i}$, we define the generalized evaluation map

$$
e v_{\underline{x}}^{\Gamma}: \mathbb{R} H^{0}\left(\Sigma ; \mathcal{L}^{d}\right) \rightarrow \mathbb{R}^{k_{1}} \times \cdots \times \mathbb{R}^{k_{m}}
$$

by

$$
s \mapsto\left(\left[s\left(x_{1}^{1}\right)\right]_{C}, \ldots,\left[s\left(x_{1}^{1}\right) \cdots s\left(x_{k_{1}}^{1}\right)\right]_{C}, \ldots,\left[s\left(x_{1}^{m}\right)\right]_{C}, \ldots,\left[s\left(x_{1}^{m}\right) \cdots s\left(x_{k_{m}}^{m}\right)\right]_{C}\right)
$$

where $C=\operatorname{graph}(s)$.

The kernel of such map is denoted by $\mathbb{R} H_{\underline{x}}^{0}$.

Remark that for points $\underline{x}=\left(x_{1}, \ldots, x_{k}\right) \in \mathbb{R} \Sigma^{k} \backslash \Delta$ outside the diagonal, the kernel of the generalized evaluation map equals the kernel of the "classical" evaluation map $s \mapsto\left(s\left(x_{1}\right), \ldots, s\left(x_{k}\right)\right)$.

Notation 5.13. In the sequel, we will only consider $k$-pointed curves of the form

$$
\left(\operatorname{graph}(s) ; s\left(x_{1}\right), \ldots, s\left(x_{k}\right)\right)
$$

for $s \in \mathbb{R} H^{0}\left(\Sigma ; \mathcal{L}^{d}\right)$ and for $x_{1}, \ldots, x_{k} \in \mathbb{R} \Sigma$. For the sake of simplicity, we will omit "graph $(s) "$ in the notation of its divided differences. For example, we will write $\left[s\left(x_{1}\right) \cdots s\left(x_{i}\right)\right]$ instead of $\left[s\left(x_{1}\right) \cdots s\left(x_{i}\right)\right]_{\operatorname{graph}(s)}$.

In the following proposition we describe the incidence variety $\mathcal{I}$ defined in Definition 5.8 in terms of divided differences.

Proposition 5.14. Let $\Gamma$ be a $k$-labelled graph, $s \in \mathbb{R} H^{0}\left(\Sigma ; \mathcal{L}^{d}\right)$ be a real section and $\underline{x}=\left(x_{1}, \ldots, x_{k}\right) \in$ $\Gamma_{d}$ be a point in the graph subset $\Gamma_{d}$. Denote by $\left\{x_{1}^{i}, \ldots, x_{k_{i}}^{i}\right\}$ the connected components of $\underline{x}$, $i \in\{1, \ldots, m\}$. Then $(s, \underline{x}) \in \mathcal{I}$ if and only if

$$
\left[s\left(x_{1}^{i}\right)\right]=0,\left[s\left(x_{1}^{i}\right) s\left(x_{2}^{i}\right)\right]=0, \ldots,\left[s\left(x_{1}^{i}\right) s\left(x_{2}^{i}\right) \cdots s\left(x_{k_{i}}^{i}\right)\right]=0
$$

for any $i \in\{1, \ldots, m\}$, that is if and only if $e v_{\underline{x}}^{\Gamma}(s)=0$ where $e v_{\underline{x}}^{\Gamma}$ is the generalized evaluation map defined in Definition 5.12.

Proof. In local coordinates the equivalence relation "having multicontact" $\left(s(\mathbb{R} \Sigma) ; s\left(x_{1}^{i}\right), \ldots, s\left(x_{k_{i}}^{i}\right)\right) \sim$ $\left(\mathbb{R} \Sigma ; x_{1}^{i}, \ldots, x_{k_{i}}^{i}\right)$ reads $\left[s\left(x_{1}^{i}\right)\right]=0,\left[s\left(x_{1}^{i}\right) s\left(x_{2}^{i}\right)\right]=0, \ldots,\left[s\left(x_{1}^{i}\right) s\left(x_{2}^{i}\right) \cdots s\left(x_{k_{i}}^{i}\right)\right]=0$. Now, having $k$-th order multi-contact is a local property, and then $\left(s(\mathbb{R} \Sigma) ; s\left(x_{1}\right), \ldots, s\left(x_{k}\right)\right) \sim\left(\mathbb{R} \Sigma ; x_{1}, \ldots, x_{k}\right)$ if and only if $\left(s(\mathbb{R} \Sigma) ; s\left(x_{1}^{i}\right), \ldots, s\left(x_{k_{i}}^{i}\right)\right) \sim\left(\mathbb{R} \Sigma ; x_{1}^{i}, \ldots, x_{k_{i}}^{i}\right)$ for any $i=1, \ldots, m$.

These equations using divided differences show that $\mathcal{I}$ is a smooth manifold:

Proposition 5.15. Let $\mathcal{L}$ be a positive real line bundle over a real Riemann surface $\Sigma$. Then, for large $d \in \mathbb{N}$, the set $\mathcal{I}$ defined in Def. 5.8 is a smooth manifold. Moreover the incidence manifold $\mathcal{I}$ defined in Section 3.2 is an open dense subset of $\mathcal{I}$, which coincide with $\pi_{\Sigma}^{-1}(\mathbb{R} \Sigma \backslash \Delta)$, where $\pi_{\Sigma}: \mathcal{I} \rightarrow \mathbb{R} \Sigma^{k}$ is the natural projection. 
Proof. We first make the following remark which is a direct consequence of the positivity of $\mathcal{L}$ and of the Riemann-Roch theorem: there exists an integer $d_{k}$ such that, for any $d \geq d_{k}$, any $k$-admissible graph $\Gamma$ and any $\underline{x}=\left(x_{1}^{1}, \ldots, x_{k_{1}}^{1}, \ldots, x_{1}^{m}, \ldots, x_{k_{m}}^{m}\right) \in \Gamma_{d}$, we can find a section $\dot{s}_{p}^{i} \in \mathbb{R} H^{0}\left(\Sigma ; \mathcal{L}^{d}\right)$ such that $e v_{\underline{x}}^{\Gamma}\left(\dot{s}_{p}^{i}\right)=(0, \ldots, 0,1,0, \ldots, 0)$, where 1 is exactly in the place corresponding to $x_{p}^{i}$.

We now prove that $\mathcal{I}$ is a smooth manifold. We have seen in Proposition 5.14 that $(s, \underline{x}) \in$ $\pi_{\Sigma}^{-1}\left(\Gamma_{d}\right) \cap \mathcal{I}$ if and only if $e v_{\underline{x}}^{\Gamma}(s)=0$. Then, we have to prove that 0 is a regular value of the map $(s, \underline{x}) \mapsto e v_{\underline{x}}^{\Gamma}(s)$. This means that, for any $\underline{\eta}=\left(\eta_{1}^{1}, \ldots, \eta_{k_{1}}^{1}, \ldots, \eta_{1}^{m}, \ldots, \eta_{k_{m}}^{m}\right) \in \mathbb{R}^{k}$, we have to find a vector $(\dot{s}, \underline{\dot{x}})=\left(\dot{s}, \dot{x}_{1}^{1}, \ldots, \dot{x}_{k_{1}}^{1}, \ldots, \dot{x}_{1}^{m}, \ldots, \bar{x}_{k_{m}}^{m}\right)$ such that

$$
\begin{gathered}
{\left[\dot{s}\left(x_{1}^{i}\right)\right]+\left[s\left(x_{1}^{i}\right) s\left(x_{1}^{i}\right)\right] \dot{x}_{1}^{i}=\eta_{1}^{i}} \\
{\left[\dot{s}\left(x_{1}^{i}\right) \dot{s}\left(x_{2}^{i}\right)\right]+\left[s\left(x_{1}^{i}\right) s\left(x_{2}^{i}\right) s\left(x_{1}^{i}\right)\right] \dot{x}_{1}^{i}+\left[s\left(x_{1}^{i}\right) s\left(x_{2}^{i}\right) s\left(x_{2}^{i}\right)\right] \dot{x}_{2}^{i}=\eta_{2}^{i}} \\
\vdots \\
{\left[\dot{s}\left(x_{1}^{i}\right) \cdots \dot{s}\left(x_{k_{i}}^{i}\right)\right]+\left[s\left(x_{1}^{i}\right) \cdots s\left(x_{k_{i}}^{i}\right) s\left(x_{1}^{i}\right)\right] \dot{x}_{1}^{i}+\cdots+\left[s\left(x_{1}^{i}\right) \cdots s\left(x_{k_{i}}^{i}\right) s\left(x_{k_{i}}^{i}\right)\right] \dot{x}_{k_{i}}^{i}=\eta_{k_{i}}^{i}}
\end{gathered}
$$

for any $i \in\{1, \ldots, m\}$.

To do this, we choose $\dot{x}_{p}^{i}=0$ for any $i \in\{1, \ldots, m\}$ and any $p \in\left\{1, \ldots, k_{i}\right\}$ and $\dot{s}=\sum_{i=1}^{m} \sum_{p=1}^{k_{i}} \eta_{p}^{i} \dot{s}_{p}^{i}$.

\subsubsection{New expressions for the density function $\mathcal{R}_{d}^{k}$}

Notation 5.16. Let $\Gamma$ be a $k$-labelled graph. We denote the restriction of the density function $\mathcal{R}_{d}^{k}$ to the graph subset $\Gamma_{d}$ by $\mathcal{R}_{d}^{\Gamma}$.

We want to estimate $\mathcal{R}_{d}^{\Gamma}$ for any $k$-labelled graph $\Gamma \in \Theta_{k}$. By the finiteness of the set of $k$-labelled graphs $\Gamma$ we obtain that Theorem 4.1 is equivalent to the following:

Proposition 5.17. Let $\Gamma$ be a k-labelled graph and $\mathcal{R}_{d}^{\Gamma}$ be the restriction of the density function $\mathcal{R}_{d}^{k}$ on the graph subset $\Gamma_{d}$ defined in Def. 5.10. Then, there exists a constant $C_{\Gamma}$ and a positive integer $d_{\Gamma}$ such that for any $d \geq d_{\Gamma}$ and for any $\underline{x}=\left(x_{1}, \ldots, x_{k}\right) \in \Gamma_{d}$

$$
\frac{1}{\sqrt{d}^{k}} \mathcal{R}_{d}^{\Gamma}(\underline{x}) \leq C_{\Gamma}
$$

Definition 5.18. Let $\Gamma$ be a $k$-labelled graph and let $\Gamma^{1}, \ldots, \Gamma^{m}$ be its connected components. We denote by $i_{1}, \ldots, i_{k_{i}}$ the vertices of $\Gamma^{i}$. We say that $\Gamma$ is an $k$-admissible graph if and only if $i_{p}<j_{q}$ for any $i<j \in\{1, \ldots, m\}$ and any $p \in\left\{1, \ldots, k_{i}\right\}, q \in\left\{1, \ldots, k_{j}\right\}$.

Remark 5.19. By Proposition 3.7 we have $\mathcal{R}_{d}^{k}=\mathcal{R}_{d}^{k} \cdot \sigma$ for any $\sigma \in \mathcal{S}_{k}$, so that it suffices to prove Proposition 5.17 for one particular element of each orbit of the action of the symmetric group $\mathcal{S}_{k}$ on the $k$-labelled graphs. As in any orbit of the action of $\mathcal{S}_{k}$ on the $k$-labelled graphs there is an admissible graph, it suffices to prove Proposition 5.17 for $k$-admissible graphs. Moreover, by Proposition 4.2, it suffices to prove Proposition 5.17 for points $\underline{x}=\left(x_{1}, \ldots, x_{k}\right)$ such that $\mathbf{d}_{h}\left(x_{i}, x_{j}\right)<\frac{\log d}{C^{\prime} \sqrt{d}}$.

Notation 5.20. Let $\Gamma$ be a $k$-admissible graph. In the sequel, we will always denote the points $\underline{x}$ in $\Gamma_{d}$ by

$$
\underline{x}=\left(x_{1}^{1}, \ldots, x_{k_{1}}^{1}, \ldots, x_{1}^{m}, \ldots, x_{k_{m}}^{m}\right)
$$

where $\left\{x_{1}^{i}, \ldots, x_{k_{i}}^{i}\right\}_{i}$ are the connected components of $\underline{x}$. As we have seen, this implies that $\mathbf{d}_{h}\left(x_{p}^{i}, x_{q}^{i}\right) \leq$ $\frac{k}{\sqrt{d}}$ and $\mathbf{d}_{h}\left(x_{p}^{i}, x_{q}^{j}\right)>\frac{1}{\sqrt{d}}$ for $i \neq j$.

The following proposition gives an explicit expression of $\mathcal{R}_{\left.d\right|_{\Gamma_{d}}}^{k} \doteqdot \mathcal{R}_{d}^{\Gamma}(\underline{x})$, where $\Gamma$ is a $k$-admissible graph. We recall that we denote by $\mathbb{R} H_{\underline{x}}^{0}$ the kernel of the generalized evaluation map defined in Definition 5.12.

Proposition 5.21. Let $\Gamma$ be a k-admissible graph. For any $\underline{x} \in \Gamma_{d}$ we have $\mathcal{R}_{d}^{\Gamma}(\underline{x})=\frac{\mathcal{N}_{d}^{\Gamma}(\underline{x})}{\mathcal{D}_{d}^{\Gamma}(\underline{x})}$, where

$$
\mathcal{N}_{d}^{\Gamma}(\underline{x})=\int_{\mathbb{R} H_{\underline{x}}^{0}} \prod_{i=1}^{m}\left|\left[s\left(x_{1}^{i}\right) s\left(x_{1}^{i}\right)\right]\right| \cdot\left|\left[s\left(x_{1}^{i}\right) s\left(x_{2}^{i}\right) s\left(x_{2}^{i}\right)\right]\right| \cdots\left|\left[s\left(x_{1}^{i}\right) \cdots s\left(x_{k_{i}}^{i}\right) s\left(x_{k_{i}}^{i}\right)\right]\right| d \mu_{\mid \mathbb{R} H_{\underline{x}}^{0}}
$$

and

$$
\mathcal{D}_{d}^{\Gamma}(\underline{x})=\left|J a c_{N}\left(e v_{\underline{x}}^{\Gamma}\right)\right| .
$$


Proof. The proof goes along the same lines as Proposition 3.9. Let $\underline{x} \in \Gamma_{d}$ be any point in $\Gamma_{d}$. Then we have seen that $(s, \underline{x}) \in \pi_{\Sigma}^{-1}\left(\Gamma_{d}\right) \cap \mathcal{I}$ if and only if $e v_{\underline{x}}^{\Gamma}(s)=0$. Differentiating this equation we see that a vector $(\dot{s}, \underline{\dot{x}})=\left(\dot{s}, \dot{x}_{1}^{1}, \ldots, \dot{x}_{k_{1}}^{1}, \ldots, \dot{x}_{1}^{m}, \ldots, \dot{x}_{k_{m}}^{m}\right)$ is in the tangent space of $(s, \underline{x})$ if and only if

$$
\begin{gathered}
{\left[\dot{s}\left(x_{1}^{i}\right)\right]+\left[s\left(x_{1}^{i}\right) s\left(x_{1}^{i}\right)\right] \dot{x}_{1}^{i}=0} \\
{\left[\dot{s}\left(x_{1}^{i}\right) \dot{s}\left(x_{2}^{i}\right)\right]+\left[s\left(x_{1}^{i}\right) s\left(x_{2}^{i}\right) s\left(x_{1}^{i}\right)\right] \dot{x}_{1}^{i}+\left[s\left(x_{1}^{i}\right) s\left(x_{2}^{i}\right) s\left(x_{2}^{i}\right)\right] \dot{x}_{2}^{i}=0} \\
\vdots \\
{\left[\dot{s}\left(x_{1}^{i}\right) \cdots \dot{s}\left(x_{k_{i}}^{i}\right)\right]+\left[s\left(x_{1}^{i}\right) \cdots s\left(x_{k_{i}}^{i}\right) s\left(x_{1}^{i}\right)\right] \dot{x}_{1}^{i}+\cdots+\left[s\left(x_{1}^{i}\right) \cdots s\left(x_{k_{i}}^{i}\right) s\left(x_{k_{i}}^{i}\right)\right] \dot{x}_{k_{i}}^{i}=0}
\end{gathered}
$$

for $i=1, \ldots, m$. This is equal to $e v_{\underline{x}}^{\Gamma}(\dot{s})+D_{\underline{x}} e v_{\underline{x}}^{\Gamma}(s) \underline{\dot{x}}=0$. Here, $D_{\underline{x}} e v_{\underline{x}}^{\Gamma}(s)$ is the derivative of $e v_{\underline{x}}(s)$ with respect to $\underline{x}$ and it is equal to the lower triangular matrix that has the following blocks on the diagonal

$$
\left[\begin{array}{cccc}
{\left[s\left(x_{1}^{i}\right) s\left(x_{1}^{i}\right)\right]} & 0 & \cdots & 0 \\
{\left[s\left(x_{1}^{i}\right) s\left(x_{2}^{i}\right) s\left(x_{1}^{i}\right)\right]} & {\left[s\left(x_{1}^{i}\right) s\left(x_{2}^{i}\right) s\left(x_{2}^{i}\right)\right]} & \cdots & 0 \\
\vdots & \vdots & \ddots & \vdots \\
{\left[s\left(x_{1}^{i}\right) \cdots s\left(x_{k_{i}}^{i}\right) s\left(x_{1}^{i}\right)\right]} & {\left[s\left(x_{1}^{i}\right) \cdots s\left(x_{k_{i}}^{i}\right) s\left(x_{2}^{i}\right)\right]} & \cdots & {\left[s\left(x_{1}^{i}\right) \cdots s\left(x_{k_{i}}^{i}\right) s\left(x_{k_{i}}^{i}\right)\right]}
\end{array}\right]
$$

for $i=1, \ldots, m$. Writing in a compact notation, we have

$$
\underline{\dot{x}}=-\left(D_{\underline{x}} e v_{\underline{x}}^{\Gamma}(s)\right)^{-1} e v_{\underline{x}}^{\Gamma}(\dot{s}) .
$$

We then deduce that the normal Jacobian that we want to compute is

$$
\left|\operatorname{Jac}_{N} \pi_{\Sigma}\right|=\frac{\left|\operatorname{Jac}_{N} e v^{\Gamma}\right|}{\left|\operatorname{Jac}_{\underline{x}} e v_{\underline{x}}^{\Gamma}(s)\right|} .
$$

We conclude by observing that $D_{\underline{x}} e v_{\underline{x}}^{\Gamma}(s)$ is triangular, so that its Jacobian is

$$
\prod_{i=1}^{m}\left|\left[s\left(x_{1}^{i}\right) s\left(x_{1}^{i}\right)\right]\right| \cdot\left|\left[s\left(x_{1}^{i}\right) s\left(x_{2}^{i}\right) s\left(x_{2}^{i}\right)\right]\right| \cdots\left|\left[s\left(x_{1}^{i}\right) \cdots s\left(x_{k_{i}}^{i}\right) s\left(x_{k_{i}}^{i}\right)\right]\right| .
$$

\subsubsection{Proof of Theorem 4.1}

Proposition 5.17 (and then Theorem 4.1, see Remark 5.19) is a consequence of the following two propositions in which we study separately the numerator $\mathcal{N}_{d}^{\Gamma}$ and the denominator $\mathcal{D}_{d}^{\Gamma}$. We denote by $C^{\prime}$ the constant that appeared in Theorem 2.2 .

Proposition 5.22. Let $\Gamma$ be a $k$-admissible graph and $\mathcal{D}_{d}^{\Gamma}$ be the function defined in Proposition 5.21. Then, there exists a positive $\epsilon_{\Gamma}>0$ and a positive integer $d_{\Gamma}$ such that for any $d \geq d_{\Gamma}$ and for any $\underline{x} \in \Gamma_{d}$ with $\mathbf{d}_{h}\left(x_{p}^{i}, x_{a}^{j}\right) \leq \frac{\log d}{C^{\prime} \sqrt{d}}$, we have

$$
\frac{1}{\sqrt{d}^{k}} \prod_{i=1}^{m} \frac{1}{\sqrt{d}^{\frac{k_{i}\left(k_{i}-1\right)}{2}}} \mathcal{D}_{d}^{\Gamma}(\underline{x})>\epsilon_{\Gamma} .
$$

Proposition 5.23. Let $\Gamma$ be a $k$-admissible graph and $\mathcal{N}_{d}^{\Gamma}$ be the function defined in Proposition 5.21. Then, there exists $M_{\Gamma}$ such that for any $d$ and for any $\underline{x} \in \Gamma_{d}$ with $\mathbf{d}_{h}\left(x_{p}^{i}, x_{a}^{j}\right) \leq \frac{\log d}{C^{\prime} \sqrt{d}}$, we have

$$
\frac{1}{d^{k}} \prod_{i=1}^{m} \frac{1}{\sqrt{d}^{\frac{k_{i}\left(k_{i}-1\right)}{2}}} \mathcal{N}_{d}^{\Gamma}(\underline{x})<M_{\Gamma} .
$$

We prove Propositions 5.22 and 5.23 respectively in Sections 5.4 and 5.5. We now prove Proposition 5.17 and Theorem 5.7. 
Proof of Proposition 5.17. As we have seen, it suffices to prove this proposition for $k$-admissible graphs and for points $\underline{x}=\left(x_{1}, \ldots, x_{k}\right)$ such that $\mathbf{d}_{h}\left(x_{i}, x_{j}\right)<\frac{\log d}{C^{\prime} \sqrt{d}}$, see Remark 5.19. The proposition follows directly from the fact that

$$
\mathcal{R}_{d}^{\Gamma}(\underline{x})=\frac{\mathcal{N}_{d}^{\Gamma}(\underline{x})}{\mathcal{D}_{d}^{\Gamma}(\underline{x})}
$$

and from Propositions 5.23 and 5.22.

Proof of Theorem 5.7. It is a consequence of the surjectivity of

$$
d \pi_{\Sigma}: T_{(s, \underline{x}} \mathcal{I} \rightarrow T_{\underline{x}} \mathbb{R} \Sigma^{k},
$$

that is equivalent to the fact that $\mathcal{D}_{d}^{\Gamma}$ is everywhere non vanishing for any graph $\Gamma \in \Theta_{k}$.

Let us prove that the density function $\mathcal{R}_{d}^{k}$ vanishes on the diagonal. Let $(s, \underline{x}) \in \mathcal{I}$ be such that $\underline{x} \in \Delta$. Then, up to an action of the symmetric group $\mathcal{S}_{k}$, we have $\underline{x}=\left(x_{1}, x_{1}, \underline{\tilde{x}}\right)$ for some $\underline{\tilde{x}} \in \mathbb{R} \Sigma^{k-2}$. Then $\mathcal{N}_{d}^{\Gamma}(\underline{x})=0$ because we integrate $\left|\left[s\left(x_{1}\right) s\left(x_{1}\right)\right]\right|$ over $\mathbb{R} H_{\underline{x}}^{0}$ (that is the kernel of the generalized evaluation map defined in Definition 5.12) and in $\mathbb{R} H_{\underline{x}}^{0}$ we have that $\left[s\left(x_{1}\right) s\left(x_{1}\right)\right]=0$. As $\mathcal{D}_{d}^{\Gamma} \neq 0$ for any $\underline{x} \in \mathbb{R} \Sigma^{k}$, we obtain $\mathcal{R}_{d}^{k}=0$ on $\Delta$.

\subsection{Estimates of the denominator $\mathcal{D}_{d}^{\Gamma}$}

This subsection is devoted to the proof of Proposition 5.22. We use the notations of Sections 5.1-5.3.

We introduce some evaluation maps $e v_{\underline{T}}^{\mathbb{C}, \Gamma}$ on the real Bargmann-Fock space $\mathbb{R} H_{L^{2}}^{0}(\mathbb{C}, \mathcal{O})$. These maps turn out to be the local models of the generalized evaluation maps $e v_{\underline{x}}^{\Gamma}$ defined in Definition 5.12. For this reason, we call them local evaluation maps. The surjectivity of the local evaluation maps on the Bargmann-Fock space will then imply the surjectivity of the generalized evaluation maps $e v_{\underline{x}}^{\Gamma}$, proving Proposition 5.22.

\subsubsection{A partition of $\mathbb{R}^{k}$}

We introduce a partition of $\mathbb{R}^{k}$ indexed by labelled graphs, as we did for $\mathbb{R} \Sigma^{k}$ in Section 5.3. Denote by $\Theta_{k}$ the set of all labelled graphs with $k$ vertices, labelled by $\{1, \ldots, k\}$. We associate a graph to every point $\left(T_{1}, \ldots, T_{k}\right) \in \mathbb{R}^{k}$. This is constructed as follows: we put an edge between the $i$-vertex and $j$-vertex if and only if the distance between $T_{i}$ and $T_{j}$ is smaller or equal than 1 . We say that $\left(T_{1}, \ldots, T_{k}\right) \in \Gamma$ if and only if its associated graph is $\Gamma$. The subset $\Gamma \subset \mathbb{R}^{k}$ is called a graph subset.

Definition 5.24. Let $\left(T_{1}, \ldots, T_{k}\right)$ be a point in $\mathbb{R}^{k}$ and $\Gamma$ be its associated graph.

- We say that $T_{i}$ and $T_{j}$ are in the same connected component if and only if the vertices $i$ and $j$ of the associated graph are in the same connected component.

- We call origin of a connected component the point of the connected component with the smallest label.

\subsubsection{A local evaluation model}

Real Bargmann-Fock space Let $\mathbb{R} H_{L^{2}}^{0}(\mathbb{C} ; \mathcal{O})$ be the space of functions of the form $f(z) e^{-\frac{|z|^{2}}{2}}$, where $f: \mathbb{C} \rightarrow \mathbb{C}$ is an holomorphic real function (that is $f(\bar{z})=f \overline{(z)})$ such that $\int_{\mathbb{C}}|f|^{2} e^{-|z|^{2}} d z d \bar{z}<$ $\infty$. We call this space the Bargmann-Fock space. It is naturally equipped with the $L^{2}$-scalar product

$$
\left\langle f e^{-\frac{|z|^{2}}{2}}, g e^{-\frac{|z|^{2}}{2}}\right\rangle=\int_{\mathbb{C}} f \bar{g} e^{-|z|^{2}} d z d \bar{z}
$$

The Bergman kernel of the orthogonal projection $L^{2}(\mathbb{C}) \rightarrow \mathbb{R} H_{L^{2}}^{0}(\mathbb{C} ; \mathcal{O})$ equals the local Bergman kernel $K_{\mathbb{C}}(x, y)=\frac{1}{\pi} e^{\frac{-\|x-y\|^{2}}{2}}$ for any $x, y \in \mathbb{C}$ (see Definition 2.5). An orthonormal basis of $\mathbb{R} H_{L^{2}}^{0}(\mathbb{C} ; \mathcal{O})$ is $\left\{\frac{z^{k}}{\sqrt{\pi} \sqrt{k !}} e^{\frac{-|z|^{2}}{2}}\right\}$. 
Local evaluation maps In the sequel we will only consider $k$-admissible graphs, see Definition 5.18. For every $k$-admissible graph $\Gamma$ and $\underline{T}=\left(T_{1}^{1}, \ldots, T_{k_{1}}^{1}, \ldots, T_{1}^{m}, \ldots, T_{k_{m}}^{m}\right) \in \Gamma$ we consider the following evaluation map:

$$
e v_{\underline{T}}^{\mathbb{C}, \Gamma}: \mathbb{R} H_{L^{2}}^{0}(\mathbb{C} ; \mathcal{O}) \rightarrow \mathbb{R}^{k}
$$

defined by

$$
f \mapsto\left(\left[f\left(T_{1}^{1}\right)\right], \ldots,\left[f\left(T_{1}^{1}\right) \cdots f\left(T_{k_{1}}^{1}\right)\right], \ldots,\left[f\left(T_{1}^{m}\right)\right], \ldots,\left[f\left(T_{1}^{m}\right) \cdots f\left(T_{k_{1}}^{m}\right)\right]\right) .
$$

The notation $\left[f\left(T_{1}^{i}\right) \ldots f\left(T_{p}^{i}\right)\right]$ stands for the divided difference $\left[f\left(T_{1}^{i}\right) \ldots f\left(T_{p}^{i}\right)\right]_{\operatorname{graph}(f)}$ in the sense of Olver, see Definition 5.5. Remark that we used the same notation in the previous section, see Notation 5.13.

Definition 5.25. We call the map $e v_{\underline{T}}^{\mathbb{C}, \Gamma}: \mathbb{R} H_{L^{2}}^{0}(\mathbb{C} ; \mathcal{O}) \rightarrow \mathbb{R}^{k}$ defined by

$$
f \mapsto\left(\left[f\left(T_{1}^{1}\right)\right], \ldots,\left[f\left(T_{1}^{1}\right) \cdots f\left(T_{k_{1}}^{1}\right)\right], \ldots,\left[f\left(T_{1}^{m}\right)\right], \ldots,\left[f\left(T_{1}^{m}\right) \cdots f\left(T_{k_{1}}^{m}\right)\right]\right)
$$

a local evaluation map.

Proposition 5.26. For any $k$-admissible graph $\Gamma \in \Theta_{k}$ and any point

$$
\underline{T}=\left(T_{1}^{1}, \ldots, T_{k_{1}}^{1}, \ldots, T_{1}^{m}, \ldots, T_{k_{m}}^{m}\right) \in \Gamma,
$$

the local evaluation map ev $v_{\underline{T}}^{\mathbb{C}, \Gamma}$ defined in Definition 5.25 is surjective.

Proof. We remark that for any $\underline{T} \in \mathbb{R}^{k} \backslash \Delta$, the map $e v_{\underline{T}}^{\mathbb{C}, \Gamma}$ is equivalent to the "classical" evaluation map $e v_{\underline{T}}: f \mapsto\left(f\left(T_{1}\right), \ldots, f\left(T_{k}\right)\right)$. It means that there exists an invertible matrix $A_{\underline{T}} \in G L_{k}(\mathbb{R})$ such that $e v_{\underline{T}}^{\mathbb{C}}=A_{\underline{T}} \cdot e v_{\underline{T}}$. The surjectivity of $e v_{\underline{T}}^{\mathbb{C}, \Gamma}$ follows from the surjectivity of $e v_{\underline{T}}$.

On the diagonal $\underline{x} \in \Delta \subset \mathbb{R}^{k}$, this new evaluation map $e v_{\underline{T}}^{\mathbb{C}, \Gamma}$ gives us more information about the higher jets of a function. If $\underline{T}=\left(T_{1}, \ldots, T_{1}, \ldots, T_{m}, \ldots, T_{m}\right)$, where $\# T_{i}=k_{i}$, then $e v_{\underline{T}}^{\mathbb{C}, \Gamma}$ is equivalent to

$$
f \mapsto\left(f\left(T_{1}\right), f^{\prime}\left(T_{1}\right), \ldots, f^{\left(k_{1}-1\right)}\left(T_{1}\right), \ldots, f\left(T_{m}\right), f^{\prime}\left(T_{m}\right), \ldots, f^{\left(k_{m}-1\right)}\left(T_{m}\right)\right) .
$$

The surjectivity of $e v_{\underline{T}}^{\mathbb{C}, \Gamma}$ follows.

\subsubsection{Local boundedness results}

The previous proposition implies that the normal Jacobian of the local evaluation map is strictly positive. We will compute the normal Jacobian of $e v_{T}^{\mathbb{C}, \Gamma}$ with respect to the metrics that we have defined, that are the $L^{2}$-scalar product on $\mathbb{R} H_{L^{2}}^{0}(\mathbb{C} ; \mathcal{O})$ and the standard metric on $\mathbb{R}^{k}$.

Proposition 5.27. Let $R>1$ be fixed and let $\Gamma$ be a $k$-admissible graph. Then there exists a positive $\epsilon_{\Gamma}>0$ such that, for every $\underline{T} \in B(0, R) \cap \Gamma$,

$$
\left|J a c_{N} e v_{\underline{T}}^{\mathbb{C}, \Gamma}\right|>\epsilon_{\Gamma}
$$

Proof. By Proposition 5.26, for any $\underline{T}=\left(T_{1}^{1}, \ldots, T_{k_{1}}^{1}, \ldots, T_{1}^{m}, \ldots, T_{k_{m}}^{m}\right) \in \Gamma$ the map $e v_{\underline{T}}^{\mathbb{C}, \Gamma}$ is surjective, so its normal Jacobian is strictly positive. By compactness of $\overline{B(0, R)}$ we can find a positive $\epsilon_{\Gamma}>0$ such that $\left|\operatorname{Jac}_{N} e v_{\underline{T}}^{\mathbb{C}, \Gamma}\right|>\epsilon_{\Gamma}$.

The following proposition shows that we can write $\operatorname{Jac}_{N} e v_{\underline{T}}^{\mathbb{C}, \Gamma}$ as a function of the distances $T_{s}^{i}-T_{t}^{i}$ between points lying in the same connected component and of the local Bergman kernel $K_{\mathbb{C}}$ (and of its derivatives).

Proposition 5.28. Let $\Gamma$ be a $k$-admissible graph. For any $\underline{T} \in \Gamma$ let $e v_{\underline{T}}^{\mathbb{C}, \Gamma}$ be the local evaluation map defined in Definition 5.25. Then the matrix associated with $\left(e v_{\underline{T}}^{\mathbb{C}, \Gamma}\right)\left(e v_{\underline{T}}^{\mathbb{C}}\right)^{*}$ is a symmetric $(k \times k)$ matrix composed by $m^{2}$ blocks, indexed by $(i, j)$ for $i, j=1, \ldots, \bar{m}$. The $(i, j)$-block is a $k_{i} \times k_{j}$ matrix, we denote the $(p, q)$-place of this block by $\left(e v_{\underline{T}}^{\mathbb{C}, \Gamma} e v_{\underline{T}}^{\mathbb{C}, \Gamma *}\right)_{\left(i_{p}, j_{q}\right)}$. Then $\left(e v_{\underline{T}}^{\mathbb{C}, \Gamma} e v_{\underline{T}}^{\mathbb{C}, \Gamma *}\right)_{\left(i_{p}, j_{q}\right)}$ is a

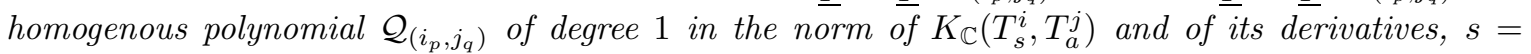
$1, \ldots, p, a=1, \ldots, q$. The coefficients of this polynomial are rational functions in $T_{s}^{i}-T_{t}^{i}, T_{a}^{j}-T_{b}^{j}$ for $1 \leq t<s \leq p, 1 \leq a<b \leq q$. 
Proof. We know that $\operatorname{Jac}_{N}\left(e v_{\underline{T}}^{\mathbb{C}, \Gamma}\right)=\operatorname{Jac}_{N}\left(\left.e v_{\underline{x}}^{\mathbb{C}, \Gamma}\right|_{\left(\operatorname{ker} e v_{\underline{T}}^{\mathbb{C}, \Gamma}\right)^{\perp}}\right)$. Let $\left\{f_{1}, \ldots, f_{k}\right\}$ be an orthonormal basis of $\left(\text { ker } e v_{\underline{T}}^{\mathbb{C}, \Gamma}\right)^{\perp}$. We compute the normal Jacobian using this basis and the canonical orthonormal basis for $\mathbb{R}^{k_{1}} \times \cdots \times \mathbb{R}^{k_{m}}$. Then the matrix of $\left.e v_{\underline{\underline{C}}}^{\mathbb{C}, \Gamma}\right|_{\left(\operatorname{ker} e v_{\underline{T}}^{\mathbb{C}, \Gamma}\right)^{\perp}}$ associated with these orthonormal basis is a square matrix whose $i$-th column equals the transpose of

$$
\left(\left[f_{i}\left(T_{1}^{1}\right)\right], \ldots,\left[f_{i}\left(T_{1}^{1}\right) \ldots f_{i}\left(T_{k_{1}}^{1}\right)\right], \ldots,\left[f_{i}\left(T_{1}^{m}\right)\right], \ldots,\left[f_{i}\left(T_{1}^{m}\right) \ldots f_{i}\left(T_{k_{m}}^{m}\right)\right]\right) .
$$

A direct computation shows that $\left(e v_{\underline{T}}^{\mathbb{C}, \Gamma}\right)\left(e v_{\underline{T}}^{\mathbb{C}, \Gamma}\right)^{*}$ is a square matrix with $m^{2}$ blocks, indexed by $(i, j)$ for $i, j=1, \ldots, m$. The $(i, j)$-block is a $k_{i} \times k_{j}$ matrix, we denote by $\left(e v_{\underline{T}}^{\mathbb{C}, \Gamma} e v_{\underline{T}}^{\mathbb{C}, \Gamma *}\right)_{\left(i_{p}, j_{q}\right)}$ the $(p, q)$-place of this block. We have that $\left(e v_{\underline{T}}^{\mathbb{C}, \Gamma} e v_{\underline{T}}^{\mathbb{C}, \Gamma *}\right)_{\left(i_{p}, j_{q}\right)}$ is equal to

$$
\sum_{l=1}^{k}\left[f_{l}\left(T_{1}^{i}\right) \ldots f_{l}\left(T_{p}^{i}\right)\right]\left[f_{l}\left(T_{1}^{j}\right) \ldots f_{l}\left(T_{q}^{j}\right)\right] .
$$

By Lemma 5.6, each term $\left[f_{l}\left(T_{1}^{i}\right) \ldots f_{l}\left(T_{p}^{i}\right)\right]\left[f_{l}\left(T_{1}^{j}\right) \ldots f_{l}\left(T_{q}^{j}\right)\right]$ is equal to

$$
\sum_{s=1}^{p} \sum_{r=0}^{\# T_{s}^{i}-1} c_{\underline{T}_{p}^{i}, s, r} f_{l}^{(r)}\left(T_{s}^{i}\right) \sum_{a=1}^{q} \sum_{h=0}^{\# T_{a}^{j}-1} c_{\underline{T}_{q}^{j}, a, h} f_{l}^{(h)}\left(T_{a}^{j}\right)
$$

where $\underline{T}_{p}^{i}=\left(T_{1}^{i}, \ldots, T_{p}^{i}\right)$ and $\underline{T}_{q}^{j}=\left(T_{1}^{j}, \ldots, T_{q}^{j}\right)$.

Interchanging the sums we obtain

$$
\sum_{s=1}^{p} \sum_{r=0}^{\# T_{s}^{i}-1} \sum_{a=1}^{q} \sum_{h=0}^{\# T_{a}^{j}-1} c_{\underline{T}_{p}^{i}, s, r} c_{\underline{T}_{q}^{j}, a, h} f_{l}^{(r)}\left(T_{s}^{i}\right) f_{l}^{(h)}\left(T_{a}^{j}\right) .
$$

Summing up to $l$ and interchanging the sums we obtain

$$
\sum_{s=1}^{p} \sum_{r=0}^{\# T_{s}^{i}-1} \sum_{a=1}^{q} \sum_{h=0}^{\# T_{a}^{j}-1} c_{\underline{T}_{p}^{i}, s, r} c_{\underline{T}_{q}^{j}, a, h} \frac{\partial^{r+h}}{\partial T^{r} \partial W^{h}} K_{\mathbb{C}}\left(T_{s}^{i}, T_{a}^{j}\right) .
$$

Remark 5.29. Remark that the Bergman kernel $K_{\mathbb{C}}(Z, W)$ only depends on the distances between $Z$ and $W$. Then, Proposition 5.28 implies that the matrix $\left(e v_{\underline{T}}^{\mathbb{C}, \Gamma}\right)\left(e v_{\underline{T}}^{\mathbb{C}}\right)^{*}$ (and then $J_{a c_{N}} e v_{\underline{T}}^{\mathbb{C}, \Gamma}$ ) only depends on the distances between the points $T_{p}^{i}$, and not on the particular position of each point.

Proposition 5.30. Let $\Gamma$ be a k-admissible graph. Then, there exists a positive $\epsilon_{\Gamma}$ such that, for any $\underline{T}=\left(T_{1}^{1}, \ldots, T_{k_{1}}^{1}, \ldots, T_{1}^{m}, \ldots, T_{k_{m}}^{m}\right) \in \Gamma$, we have

$$
\left|J a c_{N} e v_{\underline{T}}^{\Gamma}\right|>\epsilon_{\Gamma} .
$$

Proof. By induction on the number of connected components.

The case of one connected component is treated in Proposition 5.27, for $m=1$.

Consider now points

$$
\underline{T}=\left(T_{1}^{1}, \ldots, T_{k_{1}}^{1}, \ldots, T_{1}^{m}, \ldots, T_{k_{m}}^{m}\right) \in \Gamma
$$

where $\left\{T_{1}^{i}, \ldots, T_{k_{i}}^{i}\right\}$ are the connected components of $\Gamma, i \in\{1, \ldots, m\}$. We consider some connected components, say the first $l$ ones, $\Gamma^{1}, \ldots, \Gamma^{l}$ and we look at the polynomials $\mathcal{Q}_{i_{p}, j_{a}}$ defined in Proposition $5.28, i, j \in\{1, \ldots, m\} p=1, \ldots, k_{i}, a=1, \ldots, k_{j}$. For each polynomial $\mathcal{Q}_{i_{p}, j_{a}}$, we replace all the norms of $K_{\mathbb{C}}\left(T_{p}^{i}, T_{a}^{j}\right)$ and of its derivatives $i \in\{1, \ldots, l\}, j \in\{l+1, \ldots, m\}, p \in\left\{1, \ldots, k_{i}\right\}$, $a \in\left\{1, \ldots, k_{j}\right\}$, by 0 . Geometrically, we are moving the first $l$ connected components $\Gamma^{1}, \ldots, \Gamma^{l}$ to the infinity, far from the other $m-l$ components. After this operation, the determinant of the matrix $\left(e v_{T}^{\Gamma} e v_{T}^{\Gamma *}\right)$ breaks into the product of the determinants of two blocks. These blocks represent local evaluation maps with a smaller number of connected components, respectively $l$ and $m-l$. By induction, the determinant of each of these two local evaluation maps is bounded from below by a constant which depends only on $\Gamma$. 
We can apply this argument for any integer $l=1, \ldots, m-1$ and any subsets of $l$ connected components of $\left\{\Gamma^{1}, \ldots, \Gamma^{m}\right\}$. By the continuity of the determinant, we can then find two positive numbers $\delta, \epsilon>0$ such that the following property holds: every time we take a point $\underline{T} \in \Gamma$ such that the norm of $K_{\mathbb{C}}\left(T_{p}^{i}, T_{a}^{j}\right)$ is smaller than $\delta$, for some $i \neq j \in\{1, \ldots, m\}, p=1, \ldots, k_{i}, a=1, \ldots, k_{j}$, we have $\left|\operatorname{Jac}_{N} e v_{T}^{\Gamma}\right|>\epsilon$. Then, we can suppose that the norms of $K_{d}\left(T_{p}^{i}, T_{a}^{j}\right)$ are bigger than $\delta$, for any $i \neq j, i, j=1, \ldots, m, p=1, \ldots, k_{i}, a=1, \ldots, k_{j}$. In this case, there exists $R>0$ such that $\mathbf{d}\left(T_{1}^{1}, T_{p}^{i}\right)<R$ for any $i=1, \ldots, m, p=1, \ldots, k_{i}$ and then, by Proposition 5.27, we have the result.

\subsubsection{Generalized evaluation maps in normal coordinates}

We now study the surjectivity of the generalized evaluation maps $e v_{\underline{x}}^{\Gamma}: \mathbb{R} H^{0}\left(\Sigma ; \mathcal{L}^{d}\right) \rightarrow \mathbb{R}^{k_{1}} \times \cdots \times$ $\mathbb{R}^{k_{m}}$ defined in Definition 5.12. We will use the graph notation of Section 5.3.

We fix a $k$-admissible graph $\Gamma$, see Definition 5.18. Remember that this implies that for every

$$
\underline{x}=\left(x_{1}^{1}, \ldots, x_{k_{1}}^{1}, \ldots, x_{1}^{m}, \ldots, x_{k_{m}}^{m}\right)
$$

we have $\mathbf{d}_{h}\left(x_{p}^{i}, x_{q}^{i}\right) \leq \frac{k}{\sqrt{d}}$ for every $i \in\{1, \ldots, m\}$ and $1 \leq p, q \leq k_{i}$ and that $\mathbf{d}_{h}\left(x_{p}^{i}, x_{q}^{j}\right)>\frac{1}{\sqrt{d}}$ for $i \neq j$.

Around the origin $x_{1}^{1}$ we consider a real normal chart $U$, see Section 2.2.2. With a slight abuse of notation, we identify a point $x_{p}^{i}$ with its normal coordinate around $x_{1}^{1}$. We recall that with a real normal chart comes together with a real trivialization of $\mathbb{R} \mathcal{L}_{\mid U}^{d}$, see Section 2.2.2. Under these trivializations, we consider the generalized evaluation map

$$
e v_{\underline{x}}^{\Gamma}: \mathbb{R} H^{0}\left(\Sigma ; \mathcal{L}^{d}\right) \rightarrow \mathbb{R}^{k_{1}} \times \cdots \times \mathbb{R}^{k_{m}}
$$

as in Definition 5.12. On the space of real global sections $\mathbb{R} H^{0}\left(\Sigma ; \mathcal{L}^{d}\right)$ we consider the $L^{2}$-scalar product induced by the real Hermitian metric $h$, see Section 2.1.

\subsubsection{Scaled evaluation maps}

Passing to the scaled normal coordinates $T=\sqrt{d} x$ around $x_{1}^{1}$, we have new local coordinates $\left(T_{q}^{i}\right)_{i=1, \ldots m}^{q=1, \ldots k_{i}}$, where $T_{q}^{i} \in B\left(T_{1}^{i}, 1\right)$, the ball of radius 1 around $T_{1}^{i}$, see Section 2.2. We then have

$$
\left|\left[s\left(\frac{T_{1}^{i}}{\sqrt{d}}\right) \ldots s\left(\frac{T_{p}^{i}}{\sqrt{d}}\right)\right]\right|=\sqrt{d}^{p(p+1) / 2}\left|\left[s_{d}\left(T_{1}^{i}\right) \ldots s_{d}\left(T_{p}^{i}\right)\right]\right|
$$

where $s_{d}(\cdot)=s\left(\frac{\dot{\gamma}}{\sqrt{d}}\right)$.

We define the map $e v_{\underline{T}, d}^{\Gamma}: \mathbb{R} H^{0}\left(\Sigma ; L^{d}\right) \rightarrow \mathbb{R}^{k_{1}} \times \cdots \times \mathbb{R}^{k_{m}}$ by

$$
s \mapsto\left(\left[s_{d}\left(T_{1}^{1}\right)\right], \ldots,\left[s_{d}\left(T_{1}^{1}\right) \ldots s_{d}\left(T_{k_{1}}^{1}\right)\right], \ldots,\left[s_{d}\left(T_{1}^{m}\right)\right], \ldots,\left[s_{d}\left(T_{1}^{m}\right) \ldots s_{d}\left(T_{k_{m}}^{m}\right)\right]\right) .
$$

Definition 5.31. The map $e v_{\underline{T}, d}^{\Gamma}: \mathbb{R} H^{0}\left(\Sigma ; L^{d}\right) \rightarrow \mathbb{R}^{k_{1}} \times \cdots \times \mathbb{R}^{k_{m}}$ just defined is called a scaled evaluation map. We denote its normal Jacobian by

$$
D_{d}^{\Gamma}=\operatorname{Jac}_{N} e v_{\underline{T}, d}^{\Gamma} .
$$

Remark 5.32. - The main point is that scaled evalutation maps look like local evaluation maps (see Definition 5.25) when d tends to infinity. This fact will be proved in Propositions 5.33, 5.34 and 5.36 .

Proposition 5.33. Let $\Gamma$ be a k-admissible graph and $\underline{x}$ be a point in $\Gamma_{d}$. We denote by $\underline{T} \in$ $B\left(x_{1}^{1}, 1\right)^{k_{1}-1} \times \cdots \times B\left(x_{1}^{m}, 1\right)^{k_{m}-1}$ the scaled normal coordinates of $\underline{x}$ around its origin $x_{1}^{1}$. Then, we have

$$
\prod_{i=1}^{m} \frac{1}{\sqrt{d}^{\frac{k_{i}\left(k_{i}-1\right)}{2}}} \mathcal{D}_{d}^{\Gamma}(\underline{x})=D_{d}^{\Gamma}(\underline{T})
$$

where $D_{d}^{\Gamma}=J a c_{N} e v_{\underline{T}, d}^{\Gamma}$.

Proof. It is a direct consequence of the change of variables $T=\sqrt{d} x$. 


\subsubsection{Reduction to the local model}

The following result is an analogue for the scaled evaluation maps of the Proposition 5.28.

Proposition 5.34. Let $\Gamma \in \Theta_{k}$ be a $k$-admissible graph and $\underline{x}$ be a point in $\Gamma_{d}$. Let $\left(T_{1}^{i}, \ldots, T_{k_{1}}^{i}\right)$ be the scaled normal coordinates (around the origin $x_{1}^{1}$ ) of the connected component $\left(x_{1}^{i}, \ldots, x_{k_{i}}^{i}\right)$ of $\underline{x}$, $i \in\{1, \ldots, m\}$. Consider the scaled evaluation map

$$
e v_{\underline{T}, d}^{\Gamma}: \mathbb{R} H^{0}\left(\Sigma, \mathcal{L}^{d}\right) \rightarrow \mathbb{R}^{k_{1}} \times \cdots \times \mathbb{R}^{k_{m}} .
$$

Then the matrix of $e v_{\underline{T}, d}^{\Gamma} e v_{\underline{T}, d}^{\Gamma *}$ is a symmetric $(k \times k)$ matrix composed by $m^{2}$ blocks, indexed by $(i, j)$ for $i, j=1, \ldots, m$. The $(i, j)$-block is a $k_{i} \times k_{j}$ matrix, we denote the $(p, q)$-place of this block by $\left(e v_{\underline{T}, d}^{\Gamma} e v_{\underline{T}, d}^{\Gamma *}\right)_{\left(i_{p}, j_{q}\right)}$. Then $\left(e v_{\underline{T}, d}^{\Gamma} e v_{\underline{T}, d}^{\Gamma *}\right)_{\left(i_{p}, j_{q}\right)}$ is a homogenous polynomial $\mathcal{Q}_{\left(i_{p}, j_{q}\right)}$ of degree 1 in the Bergman kernel $\mathcal{K}_{d}\left(\frac{T_{s}^{i}}{\sqrt{d}}, \frac{T_{a}^{j}}{\sqrt{d}}\right)$ and in its derivatives, $s=1, \ldots, p, a=1, \ldots, q$. The coefficients of this polynomial are rational functions in $T_{s}^{i}-T_{t}^{i}$, $T_{a}^{j}-T_{b}^{j}$, for $1 \leq t<s \leq p, 1 \leq a<b \leq q$.

Proof. It is the same proof of Proposition 5.28, replacing functions $f$ by scaled sections $s_{d}$.

Remark 5.35. The homogenous polynomials $\mathcal{Q}_{\left(i_{p}, j_{q}\right)}$ of Propositions 5.34 and 5.28 are the same.

Proposition 5.36. Fix $R>1$ and let $\Gamma \in \Theta_{k}$ be a $k$-admissible graph. Consider the subset of $\Gamma_{d}$ formed by points $\underline{x}=\left(x_{1}^{1}, \ldots, x_{k_{1}}^{1}, \ldots, x_{1}^{m}, \ldots, x_{k_{m}}^{m}\right)$ with $\mathbf{d}_{h}\left(x_{p}^{i}, x_{a}^{j}\right) \leq \frac{\log d}{\sqrt{d}}$ for any $i, j \in\{1, \ldots, m\}$, $p=1, \ldots, k_{i}, a=1, \ldots, k_{j}$.

Let $\underline{T}=\left(T_{1}^{1}, \ldots, T_{k_{m}}^{m}\right) \in B(0, \log d)^{k}$ be the scaled normal coordinates of $\underline{x}$ around $x_{1}^{1}$. Then, there exists $\alpha \in(0,1)$, such that for any $d \in \mathbb{N}$ large enough and any $\underline{T}=\left(T_{1}^{1}, \ldots, T_{k_{m}}^{m}\right) \in B\left(x_{1}^{1}, \log d\right)^{k}$

$$
\frac{1}{\sqrt{d}^{k}} D_{d}^{\Gamma}(\underline{T})=J a c_{N} e v_{\underline{T}}^{\mathbb{C}, \Gamma}+O\left(\frac{1}{d^{\alpha}}\right)
$$

where ev $v_{\underline{T}}^{\mathbb{C}, \Gamma}$ is the local evaluation map defined in Definition 5.25. Moreover the error term is uniform in $T_{p}^{i} \in \bar{B}(0, \log d)$ and in $x_{1}^{1} \in \mathbb{R} \Sigma$.

Proof. This is a consequence of Proposition 5.34 and of the universality of the scaled Bergman kernel $\frac{1}{d} \mathcal{K}_{d}\left(\frac{T}{\sqrt{d}}, \frac{W}{\sqrt{d}}\right)=K_{d}(T, W)$. Indeed

$$
\frac{1}{\sqrt{d}^{k}} D_{d}^{\Gamma}(\underline{x})=\frac{1}{\sqrt{d}^{k}} \operatorname{Jac}_{N} e v_{\underline{T}, d}^{\Gamma}=\sqrt{\frac{1}{d^{k}} \operatorname{det}\left(e v_{\underline{T}, d}^{\Gamma} e v_{\underline{T}, d}^{\Gamma *}\right)} .
$$

By multilinearity of the determinant, we can multiply each entry of the matrix $\left(e v_{\underline{T}, d}^{\Gamma} e v_{\underline{T}, d}^{\Gamma *}\right)$ by $\frac{1}{d}$. We know by Proposition 5.34 that each term of this matrix $\left(e v_{\underline{T}, d}^{\Gamma} e v_{\underline{T}, d}^{\Gamma *}\right)$ is a rational function of degree 1 in $\mathcal{K}_{d}\left(x_{p}^{i}, x_{a}^{j}\right)$. This implies that each term in the matrix $\frac{1}{d}\left(e v_{\underline{T}, d}^{\Gamma} e v_{\underline{T}, d}^{\Gamma *}\right)$ is a rational function of degree 1 in $\frac{1}{d} \mathcal{K}_{d}\left(x_{p}^{i}, x_{a}^{j}\right)=\frac{1}{\pi} K_{d}\left(T_{p}^{i}, T_{a}^{j}\right)$. By Corollary 2.6, this term equals $K_{\mathbb{C}}\left(T_{p}^{i}, T_{a}^{j}\right)+O\left(\frac{1}{d^{\alpha}}\right)$. By Propositions 5.34 and 5.28 and Remark 5.35, we have the result.

Proof of Proposition 5.22. It follows from Propositions 5.36 and 5.30.

\subsection{Estimates of the numerator $\mathcal{N}_{d}^{\Gamma}$}

This subsection is devoted to the proof of the boundedness result for the numerator $\mathcal{N}_{d}^{\Gamma}$, Proposition 5.23. We start by proving the case where the graph $\Gamma$ is connected, see Proposition 5.37. The general case of $m$ connected components $\Gamma^{1}, \ldots, \Gamma^{m}$ follows by an inequality of the type $\mathcal{N}_{d}^{\Gamma} \leq \prod_{i=1}^{m} \mathcal{N}_{d}^{\Gamma^{i}}$. We use the notations of Sections 5.1-5.4, in particular we work in normal coordinates, see Section 2.2.2, and we use divided differences, see Definition 5.5.

Proposition 5.37. There exists $C>0$ such that for any $\underline{x}=\left(x_{1}, \ldots, x_{k}\right) \in \mathbb{R} \Sigma^{k}$ with $\mathbf{d}_{h}\left(x_{i}, x_{j}\right) \leq$ $\frac{k}{\sqrt{d}}$, for any $d \in \mathbb{N}$, we have

$$
\sup _{s \in S_{\underline{x}}} \frac{1}{d^{k} \sqrt{d}^{k(k-1) / 2}}\left|\left[s\left(x_{1}\right) s\left(x_{1}\right)\right]\right| \cdot\left|\left[s\left(x_{1}\right) s\left(x_{2}\right) s\left(x_{2}\right)\right]\right| \cdots\left|\left[s\left(x_{1}\right) \ldots s\left(x_{k}\right) s\left(x_{k}\right)\right]\right|<C .
$$

Here, $S_{\underline{x}}$ is the unit sphere of $\mathbb{R} H_{\underline{x}}^{0}$, the kernel of the generalized evaluation map ev $v_{\underline{x}}$ defined in Definition 5.12. 
Proof. We remember that when we use divided differences it means that we consider real normal coordinates around the origin, in this case $x_{1}$, see Section 2.2.2. We identify every point $x_{i}$ with its coordinate, in particular we still write $x_{1}$ instead of 0 .

We pass to the scaled normal coordinates that is we consider $x_{i}=\frac{T_{i}}{\sqrt{d}}$, for $T_{i} \in B\left(x_{1}, k\right)$. We then have

$$
\begin{gathered}
\left|\left[s\left(x_{1}\right) s\left(x_{1}\right)\right]\right| \cdot\left|\left[s\left(x_{1}\right) s\left(x_{2}\right) s\left(x_{2}\right)\right]\right| \cdots\left|\left[s\left(x_{1}\right) \ldots s\left(x_{k}\right) s\left(x_{k}\right)\right]\right| \\
=\sqrt{d}^{k} \sqrt{d}^{k(k-1) / 2}\left|\left[s_{d}\left(T_{1}\right) s_{d}\left(T_{1}\right)\right]\right| \cdot\left|\left[s_{d}\left(T_{1}\right) s_{d}\left(T_{2}\right) s_{d}\left(T_{2}\right)\right]\right| \cdots\left|\left[s_{d}\left(T_{1}\right) \ldots s_{d}\left(T_{k}\right) s_{d}\left(T_{k}\right)\right]\right|
\end{gathered}
$$

for any section $s$, where $s_{d}(\cdot)=s\left(\frac{\dot{\sqrt{d}}}{\sqrt{3}}\right)$. We still write $T_{1}$ instead of 0 to emphasize the fact that this rescaled local chart has $T_{1}$ as center. Consider the generalized 1-jet map $j_{\underline{x}}^{1}: \mathbb{R} H_{\underline{x}}^{0} \rightarrow \mathbb{R}^{k}$ defined by

$$
s \mapsto\left(\left[s\left(x_{1}\right) s\left(x_{1}\right)\right],\left[s\left(x_{1}\right) s\left(x_{2}\right) s\left(x_{2}\right)\right], \ldots,\left[s\left(x_{1}\right) \ldots s\left(x_{k}\right) s\left(x_{k}\right)\right]\right) .
$$

Remark that

$$
\begin{aligned}
& \sup _{s \in S_{\underline{x}}} \frac{1}{d^{k} \sqrt{d}^{k(k-1) / 2}}\left|\left[s\left(x_{1}\right) s\left(x_{1}\right)\right]\right| \cdot\left|\left[s\left(x_{1}\right) s\left(x_{2}\right) s\left(x_{2}\right)\right]\right| \cdots\left|\left[s\left(x_{1}\right) \ldots s\left(x_{k}\right) s\left(x_{k}\right)\right]\right| \\
& =\sup _{s \in S_{\underline{x}} \cap\left(\operatorname{ker} j_{\underline{x}}^{1}\right)^{\perp}} \frac{1}{d^{k} \sqrt{d}^{k(k-1) / 2}}\left|\left[s\left(x_{1}\right) s\left(x_{1}\right)\right]\right| \cdot\left|\left[s\left(x_{1}\right) s\left(x_{2}\right) s\left(x_{2}\right)\right]\right| \cdots\left|\left[s\left(x_{1}\right) \ldots s\left(x_{k}\right) s\left(x_{k}\right)\right]\right| \\
& =\sup _{s \in S_{\underline{x}} \cap\left(\operatorname{ker} j_{\underline{x}}^{1}\right)^{\perp}} \frac{1}{\sqrt{d}^{k}}\left|\left[s_{d}\left(T_{1}\right) s_{d}\left(T_{1}\right)\right]\right| \cdot\left|\left[s_{d}\left(T_{1}\right) s_{d}\left(T_{2}\right) s_{d}\left(T_{2}\right)\right]\right| \cdots\left|\left[s_{d}\left(T_{1}\right) \ldots s_{d}\left(T_{k}\right) s_{d}\left(T_{k}\right)\right]\right| .
\end{aligned}
$$

Fix an orthonormal basis $\left\{\sigma^{1}, \ldots, \sigma^{k}\right\}$ of the orthogonal of $\left(\operatorname{ker} j_{\underline{x}}^{1}\right)$.

For any $\underline{a}=\left(a_{1}, \ldots, a_{k}\right) \in S^{k-1} \subset \mathbb{R}^{k}$, write $s^{\underline{a}}=\sum_{i} a_{i} \sigma^{i}$.

Claim 1: for any $i, j \in\{1, \ldots, k\}$, the quantity

$$
\frac{1}{\sqrt{d}}\left|\left[\sigma_{d}^{j}\left(T_{1}\right) \ldots \sigma_{d}^{j}\left(T_{i}\right) \sigma_{d}^{j}\left(T_{i}\right)\right]\right|
$$

is the square root of a rational function $\mathcal{Q}_{j}$ of degree 1 in the norm of $K_{d}\left(T_{p}, T_{q}\right)$ and of its derivatives, for $p, q \in\{1, \ldots, k\}$. The coefficients of $\mathcal{Q}_{j}$ are rational functions in the distances $T_{a}-T_{s}$ between the points, for $1 \leq a \neq s \leq k$.

Remember that $\bar{\sigma}_{d}^{j}(\cdot)=\overline{\sigma^{j}}\left(\frac{\dot{\sqrt{d}}}{2}\right)$ and that we denoted $\frac{1}{d} \mathcal{K}_{d}\left(\frac{T_{i}}{\sqrt{d}}, \frac{T_{j}}{\sqrt{d}}\right)=K_{d}\left(T_{i}, T_{j}\right)$, see Section 2.2. We will conclude the proof of the proposition before proving the Claim 1. By Corollary 2.6, $K_{d}\left(T_{p}, T_{q}\right)$ converges to a local universal limit. The convergence is in $C^{2 k}$-topology and the error term is an uniform $O\left(\frac{1}{d}\right)$ that neither depend on the center $x_{1} \in \mathbb{R} \Sigma$ nor on $\left(T_{2}, \ldots, T_{k}\right) \in B\left(x_{1}, k\right)^{k-1}$. In particular, by Claim 1 , the function $\left(T_{1}, \ldots, T_{i}\right) \mapsto \frac{1}{\sqrt{d}}\left[\sigma_{d}^{j}\left(T_{1}\right) \ldots \sigma_{d}^{j}\left(T_{i}\right) \sigma_{d}^{j}\left(T_{i}\right)\right]$ does not depend on $d$, up to an uniform error term $O\left(\frac{1}{d}\right)$.

For any integer $d \in \mathbb{N}$, all points $x_{1} \in \mathbb{R} \Sigma$ and all $\left(T_{2}, \ldots, T_{k}\right) \in B\left(x_{1}, k\right)^{k-1}$ the function $S^{k-1} \rightarrow \mathbb{R}$ defined by

$$
\underline{a} \mapsto \frac{1}{\sqrt{d}^{k}}\left|\left[s \frac{a}{d}\left(T_{1}\right) s \frac{a}{d}\left(T_{1}\right)\right]\right| \cdot\left|\left[s \frac{a}{d}\left(T_{1}\right) s \frac{a}{d}\left(T_{2}\right) s \frac{a}{d}\left(T_{2}\right)\right]\right| \cdots\left|\left[s \frac{a}{d}\left(T_{1}\right) \ldots s \frac{a}{d}\left(T_{k}\right) s \frac{a}{d}\left(T_{k}\right)\right]\right|
$$

is continuous. Thanks to the Claim 1, this function does not depend on $d \in \mathbb{N}$, up to an error $O\left(\frac{1}{d}\right)$ that is uniform in $x_{1} \in \mathbb{R} \Sigma$ and in $\left(T_{2}, \ldots, T_{k}\right) \in B\left(x_{1}, k\right)^{k-1}$. By compactness of $\mathbb{R} \Sigma^{k}$ and of ${\overline{B\left(x_{1}, k\right)}}^{k-1}$ this function is bounded, then we have the result.

We will now prove the Claim 1. The proof follows the lines of Proposition 5.28. We compute the matrix of $\left(j_{\underline{x}}^{1}\right)\left(j_{\underline{x}}^{1}\right)^{*}$ with respect to the basis and in scaled normal coordinates. We have

so that

$$
j_{\underline{x}}^{1}=\left[\begin{array}{ccc}
{\left[\sigma_{d}^{1}\left(T_{1}\right) \sigma_{d}^{1}\left(T_{1}\right)\right]} & \ldots & {\left[\sigma_{d}^{k}\left(T_{1}\right) \sigma_{d}^{k}\left(T_{1}\right)\right]} \\
\vdots & \ddots & \vdots \\
{\left[\sigma_{d}^{1}\left(T_{1}\right) \ldots \sigma_{d}^{1}\left(T_{k}\right) \sigma_{d}^{1}\left(T_{k}\right)\right]} & \ldots & {\left[\sigma_{d}^{k}\left(T_{1}\right) \ldots \sigma_{d}^{k}\left(T_{k}\right) \sigma_{d}^{k}\left(T_{k}\right)\right]}
\end{array}\right]
$$

$$
\left(j_{\underline{x}}^{1}\right)\left(j_{\underline{x}}^{1}\right)_{i, j}^{*}=\sum_{l=1}^{k}\left[\sigma_{d}^{l}\left(T_{1}\right) \ldots \sigma_{d}^{l}\left(T_{i}\right) \sigma_{l}\left(T_{i}\right)\right]\left[\sigma_{d}^{l}\left(T_{1}\right) \ldots \sigma_{d}^{l}\left(T_{j}\right) \sigma_{d}^{l}\left(T_{j}\right)\right]
$$


By Proposition 5.6 we have that

$$
\left[\sigma_{d}^{1}\left(T_{1}\right) \ldots \sigma_{d}^{l}\left(T_{j}\right) \sigma_{d}^{l}\left(T_{j}\right)\right]=\sum_{h=1}^{j} \sum_{r=0}^{\# T_{h}-1} c_{\underline{T}_{j}, h, r}\left(\sigma_{d}^{l}\right)^{(r)}\left(T_{h}\right)
$$

where $c_{\underline{x}, i, r}$ is a rational function in the distances $T_{s}-T_{t}, 1 \leq s<t \leq k$. Here, $\left(\sigma_{d}^{l}\right)^{(r)}$ is the $r$-th derivative with respect to the rescaled variable $T=\sqrt{d} x$. With the same type of computation of the Proposition 5.28, we obtain that

$$
\frac{1}{d} \sum_{l=1}^{k}\left[\sigma_{d}^{l}\left(T_{1}\right) \ldots \sigma_{d}^{l}\left(T_{i}\right) \sigma_{d}^{l}\left(T_{i}\right)\right]\left[\sigma_{d}^{l}\left(T_{1}\right) \ldots \sigma_{l}\left(T_{j}\right) \sigma_{d}^{l}\left(T_{j}\right)\right]
$$

is a homogenous polynomial of degree 1 in the norm of $K_{d}\left(T_{p}, T_{q}\right)$ and of its derivatives, $p, q \in$ $\{1, \ldots, k\}$. The coefficients of this polynomial are rational functions in $T_{s}-T_{a}$ and $e^{T_{a}}$ for $1 \leq$ $a \neq s \leq k$. This implies that $\frac{1}{\sqrt{d}}\left[\sigma_{d}^{l}\left(T_{1}\right) \ldots \sigma_{d}^{l}\left(T_{i}\right) \sigma_{d}^{l}\left(T_{i}\right)\right]$ is the square root of a rational function of degree 1 in $K_{d}\left(T_{l}, T_{m}\right)$ and its derivatives, with coefficients that are rational functions in $T_{s}-T_{a}$ for $1 \leq a \neq s \leq k$, as we have claimed.

Proof of Proposition 5.23. Denote by $S_{\underline{x}}$ the unit sphere in $\mathbb{R} H_{\underline{x}}^{0}$. Passing to polar coordinates, we have

$$
\mathcal{N}_{d}^{\Gamma}=C_{r} \int_{S_{\underline{x}}} \prod_{i=1}^{m}\left|\left[s\left(x_{1}^{i}\right) s\left(x_{1}^{i}\right)\right]\right| \cdot\left|\left[s\left(x_{1}^{i}\right) s\left(x_{2}^{i}\right) s\left(x_{2}^{i}\right)\right]\right| \cdots\left|\left[s\left(x_{1}^{i}\right) \ldots s\left(x_{k_{i}}^{i}\right) s\left(x_{k_{i}}^{i}\right)\right]\right| d s
$$

for some constant $C_{r}$. The measure $d s$ is induced by the $L^{2}$-scalar product restricted to $S_{\underline{x}}$. The sphere $S_{\underline{x}}$ being compact, it suffices to bound from above the integrand function. Now,

$$
\begin{aligned}
& \sup _{s \in S_{\underline{x}}} \prod_{i=1}^{m}\left|\left[s\left(x_{1}^{i}\right) s\left(x_{1}^{i}\right)\right]\right| \cdot\left|\left[s\left(x_{1}^{i}\right) s\left(x_{2}^{i}\right) s\left(x_{2}^{i}\right)\right]\right| \cdots\left|\left[s\left(x_{1}^{i}\right) \ldots s\left(x_{k_{i}}^{i}\right) s\left(x_{k_{i}}^{i}\right)\right]\right| \\
\leq & \prod_{i=1}^{m} \sup _{s \in S_{\underline{x}^{i}}}\left|\left[s\left(x_{1}^{i}\right) s\left(x_{1}^{i}\right)\right]\right| \cdot\left|\left[s\left(x_{1}^{i}\right) s\left(x_{2}^{i}\right) s\left(x_{2}^{i}\right)\right] \cdots\right|\left[s\left(x_{1}^{i}\right) \ldots s\left(x_{k_{i}}^{i}\right) s\left(x_{k_{i}}^{i}\right)\right] \mid
\end{aligned}
$$

where $\underline{x}^{i}=\left(x_{1}^{i}, \ldots, x_{k_{i}}^{i}\right) \in \mathbb{R}^{k_{i}}$. We have then reduced the problem to the case of one connected component, that means the case where $\underline{x}=\left(x_{1}, \ldots, x_{k}\right)$ with $\mathbf{d}_{h}\left(x_{i}, x_{j}\right) \leq \frac{k}{\sqrt{d}}$ and this is exactly what we have done in Proposition 5.37.

\subsection{Off-diagonal estimates of the density function and proof of Proposition 4.7}

This subsection is devoted to the proof of Proposition 4.7. We work in the same setting of Sections 3, 4 and 5. Remember that $\mathcal{R}_{d}^{k}$ is the density function defined in Proposition 3.5.

\subsubsection{About the mean and the variance}

We recall two results which are the cases $k=1$ and $k=2$ of Theorem 1.4.

Theorem 5.38. ([11, Theorem 1.1] or [15, Theorem 1.3]) For any $x \in \mathbb{R} \Sigma$, we have

$$
\frac{1}{\sqrt{d}} \mathcal{R}_{d}^{1}(x)=\frac{1}{\sqrt{\pi}}+O\left(\frac{1}{d}\right)
$$

in the sense of currents. Moreover the error term $O\left(\frac{1}{d}\right)$ is uniform in $x \in \mathbb{R} \Sigma$.

The following is the one dimensional case of the main result of [16, Theorem 1.6].

Theorem 5.39. Under the hypothesis of Theorem 1.4, there exists a universal positive constant $M>0$

$$
\left.\frac{1}{d} \mathbb{E}\left[\nu_{s}^{2}\right](f)=\frac{1}{\pi} \int_{(x, y) \in \mathbb{R} \Sigma^{2}} f(x, y)\left|d V_{h}\right|^{2}+\frac{M}{\sqrt{d}} \int_{x \in \mathbb{R} \Sigma} f(x, x)\left|d V_{h}\right|+o\left(\frac{1}{\sqrt{d}}\right) .\right) .
$$


Moreover the error term o $\left(\frac{1}{\sqrt{d}}\right)$ is bounded from above by

$$
\|f\|_{\infty}\left(O\left(\frac{1}{\sqrt{d}^{1+\alpha}}\right)+\omega_{f}\left(\frac{1}{\sqrt{d}^{\alpha}}\right) O\left(\frac{1}{\sqrt{d}}\right)\right)
$$

for any $\alpha \in(0,1)$, where $O\left(\frac{1}{\sqrt{d}^{1+\alpha}}\right)$ and $O\left(\frac{1}{\sqrt{d}}\right)$ do not depend on $f \in C^{0}\left(\mathbb{R} \Sigma^{k}\right)$. Here, $\omega_{f}(\cdot)$ is the modulus of continuity of $f$.

The statement in [16] is a bit different, we explain why.

Proof. By definition $\operatorname{Var}\left(\nu_{s}\right)=\mathbb{E}\left[\nu_{s}^{2}\right]-\mathbb{E}\left[\nu_{s}\right]^{2}$. By Theorems 5.38 we have

$$
\frac{1}{d} \operatorname{Var}\left(\nu_{s}\right)(f)=\frac{1}{d} \mathbb{E}\left[\nu_{s}^{2}\right](f)-\frac{1}{\pi} \int_{(x, y) \in \mathbb{R} \Sigma^{2}} f(x, y)\left|\mathrm{dV}_{h}\right|^{2}+O\left(\frac{1}{d}\right) .
$$

Now, [16, Theorem 1.6], for $k=n=1$ says that

$$
\frac{1}{d} \operatorname{Var}\left(\nu_{s}\right)(f)=\frac{M}{\sqrt{d}} \int_{x \in \mathbb{R} \Sigma} f(x, x)\left|\mathrm{dV}_{h}\right|+\|f\|_{\infty}\left(O\left(\frac{1}{\sqrt{d}^{1+\alpha}}\right)+\omega_{f}\left(\frac{1}{\sqrt{d}^{\alpha}}\right) O\left(\frac{1}{\sqrt{d}}\right)\right)
$$

for a constant $M$ and for any $\alpha \in(0,1)$. In [16, Theorem 1.6] the test function $f$ is of the form $\phi_{1} \phi_{2}$, where $\phi_{i}$ is a continuous function on $\mathbb{R} \Sigma$. Their proof actually works for any continuous function $f$ over $\mathbb{R} \Sigma^{2}$. The constant $M$ is positive ([16, Theorem 1.8]) and universal, that is it does not depend on $\Sigma$.

Remark 5.40. - Using Olver multispace and divided differences coordinates as in Sections 5.35.5, we can make the proof of [16, Proposition 5.29] easier, at least for the case $k=n=1$.

This proposition is fundamental for the proof of [16, Theorem 1.6].

- The universal constant in Theorem 5.39 is not exactly the constant appearing in [16, Theorem 1.6]. This is due to a different renormalization of the curvature form $\omega$ as well as the choice of a different Gaussian measure on $\mathbb{R} H^{0}\left(\Sigma ; \mathcal{L}^{d}\right)$. However, they differ by a positive multiple.

As we work with the modified empirical measures $\tilde{\nu}_{s}^{k}$ (see Def. 3.1), we have to state an equivalent version of Theorem 5.39 for the modified second moment $\mathbb{E}\left[\tilde{\nu}_{s}^{2}\right]$.

Lemma 5.41. We follow the hypothesis of Theorem 1.3 and the notations of Def. 4.5. For every $f \in C^{0}\left(\mathbb{R} \Sigma^{k}\right)$, the following asymptotics hold:

$$
\frac{1}{d} \int_{\mathbb{R} \Sigma^{2}} f \mathcal{R}_{d}^{2}\left|d V_{h}\right|^{2}=\frac{1}{\pi} \int_{(x, y) \in \mathbb{R} \Sigma^{2}} f(x, y)\left|d V_{h}\right|^{2}+\frac{1}{\sqrt{d}} M^{\prime} \int_{x \in \mathbb{R} \Sigma} f(x, x)\left|d V_{h}\right|+o\left(\frac{1}{\sqrt{d}}\right)
$$

where $M^{\prime}=M-\frac{1}{\sqrt{\pi}}$ for $M$ as in Theorem 5.39. Moreover the error term $o\left(\frac{1}{\sqrt{d}}\right)$ is bounded from above as in Theorem 5.39.

Proof. Remember that by Proposition 3.5, we have that $\mathbb{E}\left[\widetilde{\nu}_{s}^{2}\right](f)=\int_{\mathbb{R} \Sigma^{2}} f \mathcal{R}_{d}^{2}\left|\mathrm{dV}_{h}\right|^{2}$. By Theorem 5.38 and by Definition 3.1, we obtain that

$$
\frac{1}{d} \mathbb{E}\left[\tilde{\nu}_{s}^{2}\right]=\frac{1}{d} \mathbb{E}\left[\nu_{s}^{2}\right]-\frac{1}{\pi \sqrt{d}} \int_{x \in \mathbb{R} \Sigma} f(x, x)\left|\mathrm{dV}_{h}\right|
$$

so that by Theorem 5.39,

$$
\frac{1}{d} \mathbb{E}\left[\tilde{\nu}_{s}^{2}\right](f)=\frac{1}{\pi} \int_{(x, y) \in \mathbb{R} \Sigma^{2}} f(x, y)\left|\mathrm{dV}_{h}\right|^{2}+\frac{1}{\sqrt{d}} M^{\prime} \int_{x \in \mathbb{R} \Sigma} f(x, x)\left|\mathrm{dV}_{h}\right|+o\left(\frac{1}{\sqrt{d}}\right),
$$

with $M^{\prime}=M-\frac{1}{\sqrt{\pi}}$ and with $o\left(\frac{1}{\sqrt{d}}\right)$ being as in Theorem 5.39. 


\subsubsection{Estimates of the density function}

Lemma 5.42. Under the hypothesis of Theorem 1.3, for every $f \in C^{0}\left(\mathbb{R} \Sigma^{k}\right)$, the following asymptotics hold:

$$
\frac{1}{\sqrt{d}^{k}} \int_{U_{d}^{a, b}} f \mathcal{R}_{d}^{k}\left|d V_{h}\right|^{k}=\frac{1}{\sqrt{\pi}^{k}} \int_{U_{d}^{a, b}} f\left|d V_{h}\right|^{k}+\frac{1}{\sqrt{\pi}^{k-2}} \frac{M^{\prime}}{\sqrt{d}} \int_{\mathbb{R} \Sigma^{k-1}} j_{a b}^{*} f\left|d V_{h}\right|^{k-1}+o\left(\frac{1}{\sqrt{d}}\right)
$$

where $M^{\prime}$ is as in Lemma 5.41 and the error term $o\left(\frac{1}{\sqrt{d}}\right)$ is bounded from above by

$$
\|f\|_{\infty}\left(O\left(\frac{1}{\sqrt{d}^{1+\alpha}}\right)+\omega_{f}\left(\frac{1}{\sqrt{d}^{\alpha}}\right) O\left(\frac{1}{\sqrt{d}}\right)\right)
$$

for any $\alpha \in(0,1)$, with $\omega_{f}(\cdot)$ being the modulus of continuity of $f$. Moreover the error terms $O\left(\frac{1}{\sqrt{d}^{1+\alpha}}\right)$ and $O\left(\frac{1}{\sqrt{d}}\right)$ do not depend on $f$.

Proof. By Proposition 4.2, for any $\underline{x}=\left(x_{1}, \ldots, x_{k}\right) \in U_{d}^{a b}$, we have

$$
\frac{1}{\sqrt{d}^{k}} \mathcal{R}_{d}^{k}(\underline{x})=\frac{1}{d} \mathcal{R}_{d}^{2}\left(x_{a}, x_{b}\right) \prod_{i \neq a, b} \frac{1}{\sqrt{d}} \mathcal{R}_{d}^{1}\left(x_{i}\right)+O\left(\frac{1}{d}\right)
$$

that implies

$$
\begin{gathered}
\int_{U_{d}^{a b}} f(\underline{x}) \frac{1}{\sqrt{d}^{k}} \mathcal{R}_{d}^{k}(\underline{x})\left|\mathrm{dV}_{h}\right|^{k}= \\
=\int_{U_{d}^{a b}} f(\underline{x}) \frac{1}{d} \mathcal{R}_{d}^{2}\left(x_{a}, x_{b}\right) \prod_{i=1, i \neq a, b}^{k} \frac{1}{\sqrt{d}} \mathcal{R}_{d}^{1}\left(x_{i}\right)\left|\mathrm{dV}_{h}\right|^{k}+O\left(\frac{1}{d}\right) .
\end{gathered}
$$

By Theorem 5.38 we have that $\frac{1}{\sqrt{d}} \mathcal{R}_{d}^{1}\left(x_{i}\right)=\frac{1}{\pi}+O\left(\frac{1}{d}\right)$ uniformly, so that

$$
\begin{gathered}
\int_{U_{d}^{a b}} f(\underline{x}) \frac{1}{d} \mathcal{R}_{d}^{2}\left(x_{a}, x_{b}\right) \prod_{i=1, i \neq a, b}^{k} \frac{1}{\sqrt{d}} \mathcal{R}_{d}^{1}\left(x_{i}\right)\left|\mathrm{dV}_{h}\right|^{k}+O\left(\frac{1}{d}\right)= \\
=\frac{1}{\sqrt{\pi}^{k-2}} \int_{U_{d}^{a, b}} f(\underline{x}) \frac{1}{d} \mathcal{R}_{d}^{2}\left(x_{a}, x_{b}\right)\left|\mathrm{dV}_{h}\right|^{k}+O\left(\frac{1}{d}\right)
\end{gathered}
$$

and, by Proposition 3.5 and Lemma 5.41 this is equal to

$$
\frac{1}{\sqrt{\pi}^{k}} \int_{U_{d}^{a, b}} f(\underline{x})\left|\mathrm{dV}_{h}\right|^{k}+\frac{1}{\sqrt{\pi}^{k-2}} \frac{M^{\prime}}{\sqrt{d}} \int_{\mathbb{R} \Sigma^{k-1}} j_{a b}^{*} f\left|\mathrm{dV}_{h}\right|^{k-1}+o\left(\frac{1}{\sqrt{d}}\right)
$$

where the error term is as in the statement of the present lemma.

We are now able to prove Proposition 4.7.

Proof of Proposition 4.7. It suffices to remark that $U_{d}$ is the disjoint union of the following sets (see Definition 4.5)

$$
U_{d}=\left(\mathbb{R} \Sigma^{k} \backslash \Delta_{d}\right) \cup \bigcup_{a<b}\left(U_{d}^{a, b} \backslash\left(\mathbb{R} \Sigma^{k} \backslash \Delta_{d}\right)\right) .
$$

By Lemma 4.3 and 5.42 we have the result.

\section{Complex case}

Let $(\mathcal{L}, h)$ be an ample Hermitian line bundle over a Riemann surface $\Sigma$. The number of zeros of a section $s \in H^{0}\left(\Sigma ; \mathcal{L}^{d}\right)$ is determined by the degree of $\mathcal{L}$. However, the distribution of such zeros (in the sense of currents) depends on the chosen section.

In this setting Shiffman and Zelditch proved that the zero locus of a random section $s$ of $\mathcal{L}^{d}$ becomes uniformly distributed over $\Sigma$ as $d$ grows to infinity (Theorem 1.1 of [20]). They used some estimates of the Szëgo kernels and the Poincaré-Lelong formula. In this section we will compute the higher moments of the zeros locus of a random section $s \in H^{0}\left(\Sigma ; \mathcal{L}^{d}\right)$ in the case of $\operatorname{dim} \Sigma=1$. Indeed, the methods used in this paper in the real case (Olver multispaces and Bergman kernel estimates) can be used in the same way for the complex case. 


\subsection{The complex random setting}

We work in the framework introduced by Shiffman and Zelditch in [20]. We restrict our definition for the dimension 1 .

Let $\Sigma$ be a smooth compact Riemann surface. Let $\mathcal{L} \rightarrow \Sigma$ be a holomorphic line bundle equipped with a Hermitian metric $h$ of positive curvature $\frac{i}{2 \pi} \partial \bar{\partial} \phi=\omega \in \Omega^{(1,1)}(\Sigma, \mathbb{R})$. Here, $\phi$ is the local potential of $h$. The curvature form induces a Kähler metric on $\Sigma$. Let $d x=\frac{\omega}{\int_{\Sigma} \omega}$ be the normalized volume form.

\subsubsection{The Gaussian measure on $H^{0}\left(\Sigma ; \mathcal{L}^{d}\right)$}

The Hermitian metric $h$ induces a Hermitian metric $h^{d}$ on $\mathcal{L}^{d}$ for every integer $d>0$ and also a $L^{2}$-Hermitian product on the space $H^{0}\left(\Sigma ; \mathcal{L}^{d}\right)$ of global holomorphic sections of $\mathcal{L}^{d}$ denoted by $\langle\cdot, \cdot\rangle$ and defined by

$$
\langle\alpha, \beta\rangle=\int_{\Sigma} h^{d}(\alpha, \beta) d x
$$

for any $\alpha, \beta$ in $H^{0}\left(\Sigma ; \mathcal{L}^{d}\right)$.

This Hermitian product induces a Gaussian measure on $H^{0}\left(\Sigma ; \mathcal{L}^{d}\right)$ defined by

$$
\mu(A)=\frac{1}{\pi^{N_{d}}} \int_{A} e^{-\|s\|^{2}} d s
$$

for any open subset $A \subset H^{0}\left(\Sigma ; \mathcal{L}^{d}\right)$ where $d s$ is the Lebesgue measure associated with $\langle\cdot, \cdot \cdot\rangle$ and $N_{d}=\operatorname{dim}_{\mathbb{C}} H^{0}\left(\Sigma ; \mathcal{L}^{d}\right)$.

\subsection{Olver holomorphic multispace}

Let $X$ be a complex manifold of dimension $n$. A holomorphic curve $C$ in $X$ is an analytic one-dimensional submanifold of $X$. Such a holomorphic curve is not necessarly connected, neither closed. For example a disjoint union of open disks is an holomorphic curve. A $(k+1)$-pointed holomorphic curve $\left(C ; z_{0}, \ldots, z_{k}\right)$ in $X$ is a holomorphic curve $C$ in $X$ together with $(k+1)$ not necessarly distinct points over $C$. Let $\mathcal{C}_{h}^{(k)}(X)$ be the set of all the $(k+1)$-pointed holomorphic curves in $X$. The following definition is the holomorphic analogue of the Olver multispace. We call it the Olver holomorphic multispace of a complex manifold $X$.

Definition 6.1. Two $(k+1)$-pointed holomorphic curves

$$
\mathbf{C}=\left(C ; z_{0}, \ldots, z_{k}\right) \quad \tilde{\mathbf{C}}=\left(\tilde{C} ; \tilde{z}_{0}, \ldots, \tilde{z}_{k}\right)
$$

have $k$-th order multi-contact if and only if

$$
z_{i}=\tilde{z}_{i} \text { and }\left.j_{\# i-1} C\right|_{z_{i}}=\left.j_{\# i-1} \tilde{C}\right|_{z_{i}}
$$

for each $i=0, \ldots, k$. The $k$-th order multi-space, denoted $X^{(k)}$ is the set of equivalence classes of $(k+1)$-pointed holomorphic curves in $X$ under the equivalence relation of $k$-th order multi-contact. The equivalence class of an $(k+1)$-pointed holomorphic curve $\mathbf{C}$ is called its $k$-th order multi-jet, and denoted $j_{k} \mathbf{C} \in X^{(k)}$.

As for the real case, local holomorphic coordinates of $X$ induces the holomorphic divided differences coordinates for the multispace $X^{(k)}$.

\subsection{Higher moments for random holomorphic sections}

The goal of this subsection is to prove Theorem 1.8 .

We recall our setting. Let $(\mathcal{L}, h)$ be a positive Hermitian line bundle over a Riemann surface $\Sigma$ and let $\omega$ be the Kahler form induced by $h$. We denote by $\mathrm{dV}_{\omega}^{k}$ the volume form on $\Sigma^{k}$ induced by $\omega$. For any $s \in H^{0}\left(\Sigma ; \mathcal{L}^{d}\right)$, let $C_{s}$ be the current of integration over $\{s=0\}$, that is $C_{s}(f)=\sum_{x \in\{s=0\}} f(x)$. It is known that $\frac{1}{d} \mathbb{E}\left[C_{s}\right]=\omega+O\left(\frac{1}{d}\right)$ in the sense of currents and actually that $\frac{1}{d} C_{s} \rightarrow \omega$ almost surely (see [20]). 
Proof of Theorem 1.8. The proof follows the lines of the proof of Theorem 1.4, so we only sketch the proof. By coarea formula, we can write

$$
\mathbb{E}\left[C_{s}^{k}\right](f)=\int_{\Sigma^{k}} f \mathcal{R}_{d}^{k} \mathrm{dV} V_{\omega}^{k}
$$

Define $U_{d}=\cup_{a<b} U_{d}^{a, b}$ where $U_{d}^{a, b}$ is the set

$$
U_{d}^{a, b} \doteqdot\left\{\left(x_{1}, \ldots \ldots, x_{k}\right) \in \Sigma^{k} \mid \mathbf{d}_{h}\left(x_{i}, x_{j}\right)>\frac{\log d}{C^{\prime} \sqrt{d}} \Rightarrow(i, j)=(a, b)\right\}
$$

where $C^{\prime}$ is the constant appearing in Theorem 2.2. On $U_{d}$ we have

$$
\frac{1}{d^{k}} \int_{U_{d}} f \mathcal{R}_{d}^{k} \omega_{k}=\int_{U_{d}} f \mathrm{dV}_{\omega}^{k}+\|f\|_{\infty} O\left(\frac{1}{d}\right)
$$

where $O\left(\frac{1}{d}\right)$ does not depend on $f$. This is as in Proposition 4.7. The role of Theorems 5.38 and 5.39 here is played respectively by [20, Proposition 3.2] and [21, Theorem 1.1]. By using Olver multispaces and divided differences coordinates as in Sections 5.2-5.5, we prove that $\frac{1}{d^{k}} \mathcal{R}_{d}^{k}$ is uniformly bounded, so that

$$
\frac{1}{d^{k}} \int_{\Sigma^{k} \backslash U_{d}} f \mathcal{R}_{d}^{k} \mathrm{dV}_{\omega}^{k}=\|f\|_{\infty} O\left(\frac{(\log d)^{4}}{d^{2}}\right) .
$$

Putting together the integral over $U_{d}$ and over $\Sigma^{k} \backslash U_{d}$ we obtain the result.

\section{Acknowledgements}

I would like to thank my advisor Jean-Yves Welschinger for all the fruitful discussions we had. I would also like to thank Thomas Letendre for his remarks and for his careful reading of the text. This work was performed within the framework of the LABEX MILYON (ANR-10-LABX-0070) of Université de Lyon, within the program "Investissements d'Avenir" (ANR-11-IDEX-0007) operated by the French National Research Agency (ANR).

\section{References}

[1] Michele Ancona. Expected number and distribution of critical points of real Lefschetz pencils, 2017, Preprint arXiv:1707.08490.

[2] D. Armentano, J-M. Azaïs, F. Dalmao, and J. R. León. Asymptotic variance of the number of real roots of random polynomial systems. Proc. Amer. Math. Soc., 146(12):5437-5449, 2018.

[3] Jean-Marc Azaïs and Mario Wschebor. Level sets and extrema of random processes and fields. John Wiley \& Sons, Inc., Hoboken, NJ, 2009.

[4] Robert Berman, Bo Berndtsson, and Johannes Sjöstrand. A direct approach to Bergman kernel asymptotics for positive line bundles. Ark. Mat., 46(2):197-217, 2008.

[5] Pavel Bleher, Bernard Shiffman, and Steve Zelditch. Universality and scaling of correlations between zeros on complex manifolds. Invent. Math., 142(2):351-395, 2000.

[6] Thierry Bouche. Convergence de la métrique de Fubini-Study d'un fibré linéaire positif. Ann. Inst. Fourier (Grenoble), 40(1):117-130, 1990.

[7] Xianzhe Dai, Kefeng Liu, and Xiaonan Ma. On the asymptotic expansion of Bergman kernel. J. Differential Geom., 72(1):1-41, 2006.

[8] Federico Dalmao. Asymptotic variance and CLT for the number of zeros of Kostlan Shub Smale random polynomials. C. R. Math. Acad. Sci. Paris, 353(12):1141-1145, 2015.

[9] Herbert Federer. Geometric measure theory. Die Grundlehren der mathematischen Wissenschaften, Band 153. Springer-Verlag New York Inc., New York, 1969. 
[10] Damien Gayet and Jean-Yves Welschinger. Exponential rarefaction of real curves with many components. Publ. Math. Inst. Hautes Études Sci., (113):69-96, 2011.

[11] Damien Gayet and Jean-Yves Welschinger. Betti numbers of random real hypersurfaces and determinants of random symmetric matrices. J. Eur. Math. Soc. (JEMS), 18(4):733-772, 2016.

[12] Hamid Hezari, Casey Kelleher, Shoo Seto, and Hang Xu. Asymptotic expansion of the Bergman kernel via perturbation of the Bargmann-Fock model. J. Geom. Anal., 26(4):2602-2638, 2016.

[13] M. Kac. A correction to "On the average number of real roots of a random algebraic equation.". Bull. Amer. Math. Soc., 49:938, 1943.

[14] E. Kostlan. On the distribution of roots of random polynomials. In From Topology to Computation: Proceedings of the Smalefest (Berkeley, CA, 1990), pages 419-431. Springer, New York, 1993.

[15] Thomas Letendre. Expected volume and Euler characteristic of random submanifolds. J. Funct. Anal., 270(8):3047-3110, 2016.

[16] Thomas Letendre and Martin Puchol. Variance of the volume of random real algebraic submanifolds II, 2017, Preprint arXiv:1707.09771.

[17] Xiaonan Ma and George Marinescu. Holomorphic Morse inequalities and Bergman kernels, volume 254 of Progress in Mathematics. Birkhäuser Verlag, Basel, 2007.

[18] Xiaonan Ma and George Marinescu. Exponential estimate for the asymptotics of Bergman kernels. Math. Ann., 362(3-4):1327-1347, 2015.

[19] Peter J. Olver. Geometric foundations of numerical algorithms and symmetry. Appl. Algebra Engrg. Comm. Comput., 11(5):417-436, 2001. Special issue "Computational geometry for differential equations".

[20] Bernard Shiffman and Steve Zelditch. Distribution of zeros of random and quantum chaotic sections of positive line bundles. Comm. Math. Phys., 200(3):661-683, 1999.

[21] Bernard Shiffman and Steve Zelditch. Number variance of random zeros on complex manifolds, II: smooth statistics. Pure Appl. Math. Q., 6(4, Special Issue: In honor of Joseph J. Kohn. Part 2):1145-1167, 2010.

[22] M. Shub and S. Smale. Complexity of Bezout's theorem. II. Volumes and probabilities. In Computational algebraic geometry (Nice, 1992), volume 109 of Progr. Math., pages 267-285. Birkhäuser Boston, Boston, MA, 1993.

[23] Gang Tian. On a set of polarized Kähler metrics on algebraic manifolds. J. Differential Geom., $32(1): 99-130,1990$. 